Shane Cooper and Anton Savostianov*

\title{
Homogenisation with error estimates of attractors for damped semi-linear anisotropic wave equations
}

https://doi.org/10.1515/anona-2020-0024

Received September 16, 2018; accepted March 1, 2019.

Abstract: Homogenisation of global $\mathcal{A}^{\varepsilon}$ and exponential $\mathcal{M}^{\varepsilon}$ attractors for the damped semi-linear anisotropic wave equation $\partial_{t}^{2} u^{\varepsilon}+y \partial_{t} u^{\varepsilon}-\operatorname{div}\left(a\left(\frac{x}{\varepsilon}\right) \nabla u^{\varepsilon}\right)+f\left(u^{\varepsilon}\right)=g$, on a bounded domain $\Omega \subset \mathbb{R}^{3}$, is performed. Order-sharp estimates between trajectories $u^{\varepsilon}(t)$ and their homogenised trajectories $u^{0}(t)$ are established. These estimates are given in terms of the operator-norm difference between resolvents of the elliptic operator $\operatorname{div}\left(a\left(\frac{x}{\varepsilon}\right) \nabla\right)$ and its homogenised limit $\operatorname{div}\left(a^{h} \nabla\right)$. Consequently, norm-resolvent estimates on the Hausdorff distance between the anisotropic attractors and their homogenised counter-parts $\mathcal{A}^{0}$ and $\mathcal{M}^{0}$ are established. These results imply error estimates of the form $\operatorname{dist}_{X}\left(\mathcal{A}^{\varepsilon}, \mathcal{A}^{0}\right) \leq C \varepsilon^{\varkappa}$ and $\operatorname{dist}_{X}^{S}\left(\mathcal{M}^{\varepsilon}, \mathcal{M}^{0}\right) \leq C \varepsilon^{\varkappa}$ in the spaces $X=L^{2}(\Omega) \times H^{-1}(\Omega)$ and $X=\left(C^{\beta}(\bar{\Omega})\right)^{2}$. In the natural energy space $\mathcal{E}:=H_{0}^{1}(\Omega) \times L^{2}(\Omega)$, error estimates $\operatorname{dist}_{\mathcal{E}}\left(\mathcal{A}^{\varepsilon}, \mathrm{T}_{\mathcal{E}} \mathcal{A}^{0}\right) \leq C \sqrt{\varepsilon}{ }^{\varkappa}$ and $\operatorname{dist}_{\mathcal{E}}^{s}\left(\mathcal{M}^{\varepsilon}, \mathrm{T}_{\varepsilon} \mathcal{M}^{0}\right) \leq C \sqrt{\varepsilon}{ }^{\varkappa}$ are established where $\mathrm{T}_{\varepsilon}$ is first-order correction for the homogenised attractors suggested by asymptotic expansions. Our results are applied to Dirchlet, Neumann and periodic boundary conditions.

Keywords: damped wave equation, global attractor, exponential attractor, homogenisation, homogenization, error estimates

MSC: 35B40, 35B45, 35L70, 35B27

\section{Introduction}

In this article we consider the following damped semi-linear wave equation in a bounded smooth domain $\Omega \subset \mathbb{R}^{3}$ with rapidly oscillating coefficients:

$$
\left\{\begin{array}{l}
\partial_{t}^{2} u^{\varepsilon}+y \partial_{t} u^{\varepsilon}-\operatorname{div}\left(a\left(\frac{x}{\varepsilon}\right) \nabla u^{\varepsilon}\right)+f\left(u^{\varepsilon}\right)=g(x), \quad x \in \Omega, t \geq 0, \\
\left.\left(u^{\varepsilon}, \partial_{t} u^{\varepsilon}\right)\right|_{t=0}=\xi,\left.\quad u^{\varepsilon}\right|_{\partial \Omega}=0 .
\end{array}\right.
$$

Such equations appear, for example, in the context of non-linear ascoustic oscillations in periodic composite media (see for example [1]).

For fixed $\varepsilon>0$, the long-time behaviour of $u^{\varepsilon}$ has been intensively studied in many works under various assumptions on the non-linearity $f$ and force $g$. In the context of dissipative PDEs the long-time dynamics can be studied in terms of global attractors. Intuitively speaking, the global attractor is a compact subset of the infinite-dimensional phase space which attracts all trajectories that originate from bounded regions of phase space. Therefore, the global attractor is in some sense a 'much smaller' subset of phase space that characterises the long-time dynamics of the system (see for example [2-7]).

Shane Cooper, Durham University, Department of Mathematical Sciences, Durham, DH1 3LE, United Kingdom, E-mail:shane.a.cooper@durham.ac.uk

*Corresponding Author: Anton Savostianov, Durham University, Department of Mathematical Sciences, Durham, DH1 3LE, United Kingdom, E-mail: anton.savostianov@durham.ac.uk 
It is well-known that for suitable assumptions on the non-linearity (cf. [2, 7]) that problem (0.1) possesses a global attractor $\mathcal{A}^{\varepsilon}$ and an important question to ask, from the point of view of applications, is about the asymptotic structure, with respect to $\varepsilon$, of the global attractor $\mathcal{A}^{\varepsilon}$ in the limit of small $\varepsilon$. Asymptotics for global attractors have been studied, in the context of reaction diffusion equations and the damped wave equation, with respect to 'lower-order' rapid spatial oscillations in the dampening, non-linearity and/or forces $g$ (see [8-11]). Yet surprisingly, to the best knowledge of the authors, little or no work has been performed on the asymptotics of attractors for hyperbolic dissipative systems with 'higher-order' rapid spatial oscillations such as in (0.1). We mention here the works [12] that perform a quantitative analysis of the asymptotics of global attractors in the context of reaction diffusion equations. We also mention the works $[13,14]$ that determine the limit-behaviour of global attractors, in the context of reaction-diffusion and hyperbolic equations, for a particular choice of rapidly oscillating coefficients that degenerate in the limit of small period. Aside from the very limited amount of work done on the asymptotics of global attractors for dissipative PDEs with rapid oscillations, no work has been done on the asymptotics of exponential attractors. This article is dedicated to performing these studies for problems of the form (0.1).

In this article we aim to study the long-time behaviour of trajectories $u^{\varepsilon}$ to $(0.1)$, for small parameter $\varepsilon$, from the point of view of homogenisation theory. In homogenisation theory, the mapping

$$
A_{\varepsilon} u:=-\operatorname{div}(a(\dot{\bar{\varepsilon}}) \nabla u),
$$

for periodic uniformly elliptic and bounded coefficients $a(\cdot)$, is well-known to converge (in an appropriate sense) in the limit of small $\varepsilon$ to

$$
A_{0} u:=-\operatorname{div}\left(a^{h} \nabla u\right),
$$

where $a^{h}$ is the 'effective' or 'homogenised' constant-coefficient matrix associated to $a(\cdot)$ (see for example [15] and references therein). As such, it is natural to compare the long-time dynamics of $u^{\varepsilon}$ to the long-time dynamics of $u^{0}$ the solution to homogenised problem

$$
\left\{\begin{array}{l}
\partial_{t}^{2} u^{0}+y \partial_{t} u^{0}-\operatorname{div}\left(a^{h} \nabla u^{0}\right)+f\left(u^{0}\right)=g(x), \quad x \in \Omega, t \geq 0, \\
\left.\left(u^{0}, \partial_{t} u^{0}\right)\right|_{t=0}=\xi,\left.\quad u^{0}\right|_{\partial \Omega}=0 .
\end{array}\right.
$$

Homogenisation theory has been studied intensively since the 1970's and amongst the extensive works we focus on works related to quantitative estimates of the form

$$
\left\|A_{\varepsilon}^{-1}-A_{0}^{-1}\right\|_{\mathcal{L}\left(L^{2}(\Omega)\right)} \leq C \varepsilon,
$$

where the mappings have been equipped with appropriate boundary conditions. Such (sharp) order- $\varepsilon$ results, that are now standard, has been proved by various authors using various techniques (see the monograph [16] for a review of some of these techniques). We mention here the results of particular interest to our article; in the case of bounded domain with Dirichlet or Neumann boundary conditions the order-sharp estimates were proved for the first time in $[17,18]$ and utilised the (order-sharp) estimate proved in $[19,20]$ for the whole space (and periodic torus).

While some work has been done to provide order-sharp operator estimates for individual trajectories in the parabolic (cf. [15, 16]) or hyperbolic settings (for smooth enough initial data) (cf. [21-24]), no work is done on providing order-sharp operator estimates for attractors in dissipative PDEs.

Our first main result is the following estimate ${ }^{1}$ between the global attractors $\mathcal{A}^{\varepsilon}$ and $\mathcal{A}^{0}$, associated to problem (0.1) and (0.2) respectively, in the energy spaces $\mathcal{E}^{-1}:=L^{2}(\Omega) \times H^{-1}(\Omega)$ and $\left(C^{\beta}(\bar{\Omega})\right)^{2}$ (see Theorem 4.3 and Corollary 4.1):

$$
\begin{aligned}
& \operatorname{dist}_{\mathcal{E}^{-1}}\left(\mathcal{A}^{\varepsilon}, \mathcal{A}^{0}\right) \leq C\left\|A_{\varepsilon}^{-1}-A_{0}^{-1}\right\|_{\mathcal{L}\left(L^{2}(\Omega)\right)}^{\varkappa}, \\
& \operatorname{dist}_{\left(C^{\beta}(\bar{\Omega})\right)^{2}}\left(\mathcal{A}^{\varepsilon}, \mathcal{A}^{0}\right) \leq C\left\|A_{\varepsilon}^{-1}-A_{0}^{-1}\right\|_{\mathcal{L}\left(L^{2}(\Omega)\right)}^{\theta \varkappa},
\end{aligned}
$$

1 Here $\operatorname{dist}_{X}(A, B)$ denotes the one-sided Hausdorff metric between sets $A$ and $B$ in the strong topology of $X$. 
for some $\varkappa, \theta \in(0,1)$. Upon combining this result with the operator estimate $(0.3)$ gives the desired error estimates between global attractors.

The above inequality is new in the homogenisation theory of attractors. Moreover, this result is important from the general perspective as it establishes the upper semi-continuity of global attractors of the damped wave equation in terms of the elliptic part of the PDE. Indeed, in the proof of this result we do not use the asymptotic structure in $\varepsilon$ of $S_{\varepsilon}(t)$ in terms of $S_{0}(t)$. The arguments are purely operator-theoretic in nature and only require that the elliptic operator is self-adjoint and boundedly invertible (see Section 4). In particular, if $A_{\varepsilon}$ and $A_{0}$ were positive elliptic operators $A=\operatorname{div}(a \nabla)$ and $B=\operatorname{div}(b \nabla)$ for two different matrices $a$ and $b$, the above continuity result still holds. Additionally, the same can be said for different boundary conditions: one can replace Dirichlet boundary conditions with other types of boundary conditions under the sole requirement that $A=\operatorname{div}(a \nabla)$ defines a self-adjoint operator in $L^{2}(\Omega)$ (see Section 7 for details).

Let us say a few words on the method of proof of (0.4). This result is essentially proved by establishing the following (sharp) estimate between trajectories $u^{\varepsilon}(t)$ and $u^{0}(t)$ for initial data in $\mathcal{A}^{\varepsilon}$ (Theorem 4.2):

$$
\left\|u^{\varepsilon}(t)-u^{0}(t)\right\|_{L^{2}(\Omega)}+\left\|\partial_{t} u^{\varepsilon}(t)-\partial_{t} u^{0}(t)\right\|_{H^{-1}(\Omega)} \leq M e^{K t}\left\|A_{\varepsilon}^{-1}-A_{0}^{-1}\right\|_{\mathcal{L}\left(L^{2}(\Omega)\right)}, \quad t \geq 0 .
$$

Then, to prove (0.4), we combine this novel estimate with the exponential attraction property of $\mathcal{A}^{0}$ which is known to hold 'generically' on an open dense subset of forces $g$ :

$$
\left\{\begin{array}{l}
\exists \sigma>0 \text { such that for every bounded set } B \subset \mathcal{E} \text { the following estimate holds: } \\
\operatorname{dist}_{\mathcal{E}}\left(S_{0}(t) B, \mathcal{A}^{0}\right) \leq M\left(\|B\|_{\mathcal{E}}\right) e^{-\sigma t}, \quad t \geq 0 .
\end{array}\right.
$$

Notice that estimate (0.5) is optimal; indeed, upon substituting the right-hand side with $\varepsilon$ we arrive at the expected order-sharp estimates in $\varepsilon$ (just as in the elliptic case (0.3)).

Aside from (0.4), a natural question to ask is if we can compare the global attractors in the energy space $\mathcal{E}:=H_{0}^{1}(\Omega) \times L^{2}(\Omega)$. In general estimates of the form (0.4) are not to be expected in $\mathcal{E}$ and this is due to the fact that, on the level of asymptotic expansions, the trajectories $\nabla u^{\varepsilon}(t)$ are not close to $\nabla u^{0}(t)$ but instead are close to

$$
\mathcal{T}_{\varepsilon} u^{0}(t, x):=u^{0}(t, x)+\varepsilon \sum_{i=1}^{3} N_{i}\left(\frac{x}{\varepsilon}\right) \partial_{x_{i}} u^{0}(t, x)
$$

Here $N_{i}$ are the solutions to the so-called auxiliary cell problem (see Section 1). Indeed, in Homogenisation theory it is known that (0.3) does not generally hold in $H^{1}(\Omega)$ but rather the following 'corrector' estimate

$$
\left\|A_{\varepsilon}^{-1} g-\mathcal{T}_{\varepsilon} A_{0}^{-1} g\right\|_{H^{1}(\Omega)} \leq C \sqrt{\varepsilon}\|g\|_{L^{2}(\Omega)},
$$

holds (cf. the above citations on error estimates in homogenisation of elliptic systems). For this reason, we introduce the notion of correction to attractors:

$$
\mathrm{T}_{\varepsilon} \xi:=\left(\mathcal{T}_{\varepsilon} \xi^{1}, \xi^{2}\right), \quad \xi=\left(\xi^{1}, \xi^{2}\right) \in \mathcal{A}^{0},
$$

and our next main result is the following corrector estimate (Theorem 5.3):

$$
\operatorname{dist}_{\varepsilon}\left(\mathcal{A}^{\varepsilon}, \mathrm{T}_{\varepsilon} \mathcal{A}^{0}\right) \leq C \sqrt{\varepsilon} \varkappa .
$$

To the best of our knowledge, in all previous works, no corrector estimates were provided in the homogenisation of attractors. To prove this result we naturally aim to establish an inequality of the form:

$$
\left\|u^{\varepsilon}(t)-\mathcal{T}_{\varepsilon} u^{0}(t)\right\|_{H_{0}^{1}(\Omega)} \leq M e^{K t} \sqrt{\varepsilon}, \quad t \geq 0,
$$

for initial data $\xi \in \mathcal{A}^{\varepsilon}$. It turns out that for such initial data the trajectory $u^{0}(t)$ does not contain enough regularity for such a result to hold. This issue is due to the hyperbolic nature of the problem and does not appear, for example, in the context of parabolic equations. To overcome this issue we introduce specially prepared initial data $\xi_{0}$ for the trajectory $u^{0}$ as follows: $\xi_{0}^{1} \in H_{0}^{1}(\Omega)$ is the solution to

$$
\operatorname{div}\left(a^{h} \nabla \xi_{0}^{1}\right)=\operatorname{div}\left(a(\dot{\bar{\varepsilon}}) \nabla \xi^{1}\right) \quad \text { in } \Omega .
$$


Then, for such a choice of initial data, we readily establish inequality (0.7) (Theorem 5.2 and Corollary 5.1) and consequently prove (0.6). Such initial data was originally introduced in [25] in the homogenisation (without error estimates) of the linear wave equation.

An important question from the point of view of applications is whether or not the estimates $(0.4),(0.6)$ hold in the symmetric Hausdorff distance

$$
\operatorname{dist}^{s}\left(\mathcal{A}^{\varepsilon}, \mathcal{A}^{0}\right)=\max \left\{\operatorname{dist}\left(\mathcal{A}^{\varepsilon}, \mathcal{A}^{0}\right), \operatorname{dist}\left(\mathcal{A}^{0}, \mathcal{A}^{\varepsilon}\right)\right\} .
$$

To prove this one would need to show that for sufficiently small $\varepsilon$ the global attractor $\mathcal{A}^{\varepsilon}$ is in fact (generically) an exponential attractor with exponent, and set of generic forces, independent of $\varepsilon$. Such a result seems reasonable from the perspective of considering $\mathcal{A}^{\varepsilon}$ to be an 'appropriate' perturbation of the global attractor $\mathcal{A}^{0}$ and applying the theory of regular attractors, see for example $[2,26]$. Such a result has yet to be established and we intend to carry out this study in future work.

That being said, it is known that, in general, global attractors are not continuous (in the symmetric Hausdorff distance) under perturbations and that the rate of attraction can be arbitrarily slow. For this reason the theory of exponential attractors was developed; such exponential attractors are known to be stable under perturbations and attract bounded sets exponentially fast in time. Importantly, exponential attractors also occupy 'small' subsets of phase space in the sense that they have finite fractal dimension, cf. [27-30].

Motivated by the above discussion, and the desire for estimates in the symmetric Hausdorff distance, we also study the relationship between exponential attractors associated to problems (0.1) and (0.2). In fact we construct exponential attractors $\mathcal{M}^{\varepsilon}$ and $\mathcal{M}{ }^{0}$ whose (finite) fractal dimension and exponents of attraction are independent of $\varepsilon$, and we determine the following analogues of (0.4) and (0.6) in the symmetric distance (Theorem 6.1, Corollary 6.1 and Theorem 6.3):

$$
\left\{\begin{array}{l}
\operatorname{dist}_{\mathcal{E}^{-1}}^{S}\left(\mathcal{M}^{\varepsilon}, \mathcal{M}^{0}\right) \leq C\left\|A_{\varepsilon}^{-1}-A_{0}^{-1}\right\|_{\mathcal{L}\left(L^{2}(\Omega)\right)}^{\varkappa}, \\
\operatorname{dist}_{\left(C^{\beta}(\bar{\Omega})\right)^{2}}^{s}\left(\mathcal{M}^{\varepsilon}, \mathcal{M}^{0}\right) \leq C\left\|A_{\varepsilon}^{-1}-A_{0}^{-1}\right\|_{\mathcal{L}\left(L^{2}(\Omega)\right)} \|^{2}, \\
\operatorname{dist}_{\mathcal{E}}^{s}\left(\mathcal{M}^{\varepsilon}, \mathrm{T}_{\mathcal{E}} \mathcal{M}^{0}\right) \leq C \sqrt{\varepsilon} .
\end{array}\right.
$$

To establish the last inequality above we developed further (in Theorem 6.4) the known abstract construction of exponential attractors of semi-groups to include the case of semi-groups that admit asymptotic expansions (i.e. 'corrections' such as $\mathcal{T}_{\varepsilon}$ ).

We end the introduction with some words on the structure of this article. In Section 1, we formulate precise assumptions on the non-linearity $f$ and the elliptic part of (0.1), (0.2). Also, we recall relevant known well-posedness results as well as results on the existence of global attractors associated with (0.1), (0.2). For the reader's convenience, details on the corresponding attractor theory is provided in Appendix A. In Section 2, for the dynamical systems generated by problems (0.1), (0.2), we establish existence and smoothness results for an attracting set (which contains the global attractors). These results will be crucial in justifying error estimates between anisotropic and homogenised attractors. In Section 3, we establish the convergence, in the limit of $\varepsilon \rightarrow 0$, of the anisotropic global attractor $\mathcal{A}^{\varepsilon}$ to the homogenised attractor $\mathcal{A}^{0}$ in the spaces $\mathcal{E}^{-1}$ and $\left(C^{\beta}(\bar{\Omega})\right)^{2}$. In Section 4, we derive the central (order-sharp) estimate (0.5) on the difference between trajectories $u^{\varepsilon}(t)$ and $u^{0}(t)$ of the corresponding anisotropic and homogenised problems. Then, based on this, we demonstrate the quantitative estimates (0.4) on the distance between global attractors $\mathcal{A}^{\varepsilon}$ and $\mathcal{A}^{0}$. Estimate (0.6) between the global attractor $\mathcal{A}^{\varepsilon}$ and first-order correction $\mathrm{T}_{\varepsilon} \mathcal{A}^{0}$ in the energy space $\varepsilon$ is proved in Section 5. Section 6 is devoted to exponential attractors $\mathcal{M}^{\varepsilon}, \mathcal{M}^{0}$ associated with problems (0.1), (0.2) and consists of two parts. In Subsection 6.1, existence of the exponential attractors is proved and estimates (0.8) in $\mathcal{E}^{-1}$ and $\left(C^{\beta}(\bar{\Omega})\right)^{2}$ are obtained. The results in this section rely on a variant of a standard abstract result on the construction of exponential attractors; this construction is included in Appendix B. In Subsection 6.2, we compare the distance between the exponential attractor $\mathcal{M}^{\varepsilon}$ and the first-order correction $\mathrm{T}_{\mathcal{E}} \mathcal{M}^{0}$ in the energy space $\mathcal{E}$. Subsection 6.2 rests on a new abstract theorem, presented in Appendix C, which compares the distance between exponential attractors which admit correction. We discuss, and prove the corresponding results for the cases of Neumann and periodic boundary conditions in Section 7. Some refinements of the 
results obtained in Sections 2-4 related to boundary corrections in homogenisation theory are the subject of Appendix D.

\section{Notations}

We document here notations frequently used throughout the article. The $L^{2}(\Omega)$ inner product is given by $(u, v):=\int_{\Omega} u(x) v(x) d x$, with norm denoted by $\|u\|:=(u, u)^{1 / 2}$ for $u, v \in L^{2}(\Omega)$. We frequently consider initial data in the energy spaces $\mathcal{E}^{-1}:=L^{2}(\Omega) \times H^{-1}(\Omega)$, and $\mathcal{E}:=H_{0}^{1}(\Omega) \times L^{2}(\Omega)$. These spaces are equipped with norms whose squares are given as $\|\xi\|_{\mathcal{E}^{-1}}^{2}:=\left\|\xi^{1}\right\|^{2}+\left\|\xi^{2}\right\|_{H^{-1}(\Omega)}^{2}$ and $\|\xi\|_{\mathcal{E}}^{2}:=\left\|\nabla \xi^{1}\right\|^{2}+\left\|\xi^{2}\right\|^{2}$ for admissible pairs ${ }^{2} \xi=\left(\xi^{1}, \xi^{2}\right)$. For any function $z(t)$ we set $\xi_{z}(t)$ to be the pair $\left(z(t), \partial_{t} z(t)\right)$ where $\partial_{t} z$ denotes the distributional (time) derivative. For a Banach space $E, B_{E}(0, r)$ denotes the ball centered at 0 of radius $r$ in $E$; the symbol $[\cdot]_{E}$ denotes the closure in $E$; the one-sided and symmetric Hausdorff distances between two sets $A, B \subset E$ are respectively defined as $\operatorname{dist}_{E}(A, B):=\sup _{a \in A} \inf _{b \in B}\|a-b\|_{E}$ and $\operatorname{dist}_{E}^{S}(A, B):=\max \left\{\operatorname{dist}_{E}(A, B), \operatorname{dist}_{E}(B, A)\right\}$. The standard Euclidean basis is denoted by $\left\{e_{k}\right\}_{k=1}^{3}$.

\section{Preliminaries}

Throughout the article, unless stated otherwise, we adopt the convention that $M$ and $K$ denote generic constants whose precise value may vary from line to line.

For a given matrix $a(\cdot)=\left\{a_{i j}(\cdot)\right\}_{i, j=1}^{3}$ we denote by $a^{h}=\left\{a_{i j}^{h}\right\}_{i, j=1}^{3}$ the homogenised matrix corresponding to $a(\cdot)$ whose constant coefficients are given by the formula

$$
a_{i j}^{h}:=\int_{Q}\left(a_{i j}(y)+\sum_{k=1}^{3} a_{i k}(y) \partial_{y_{k}} N_{j}(y)\right) d y .
$$

Here $N_{i}, i \in\{1,2,3\}$, is the solution to the so-called cell problem:

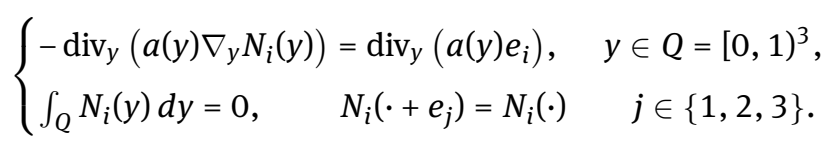

It is well-known that if $a(\cdot)$ is symmetric, bounded and uniformly elliptic, then so is $a^{h}$ with the exact same bounds (see for example [15, Section 1]). Furthermore, as $a^{h}$ is constant it is clearly periodic. Consequently, both problem (0.1) and (0.2) are problems of the form

$$
\left\{\begin{array}{l}
\partial_{t}^{2} u+y \partial_{t} u-\operatorname{div}(a \nabla u)+f(u)=g(x), \quad x \in \Omega, t \geq 0, \\
\left.\left(u, \partial_{t} u\right)\right|_{t=0}=\xi,\left.\quad u\right|_{\partial \Omega}=0,
\end{array}\right.
$$

with the same generic assumptions on coefficients, forces and non-linearity; we collect these assumptions together here:

$$
\left\{\begin{array}{l}
\text { Let } \Omega \subset \mathbb{R}^{3} \text { be a bounded smooth domain, } g \in L^{2}(\Omega), a(\cdot)=\left\{a_{i j}(\cdot)\right\}_{i, j=1}^{3} \text { satisfying } \\
a_{i j} \in L^{\infty}\left(\mathbb{R}^{3}\right), \quad a_{i j}=a_{j i}, \quad a_{i j}\left(\cdot+e_{k}\right)=a_{i j}(\cdot), \quad i, j, k \in\{1,2,3\}, \\
\& \quad v|\eta|^{2} \leq a(y) \eta \cdot \eta \leq v^{-1}|\eta|^{2}, v>0, \quad \forall y \in \mathbb{R}^{3}, \forall \eta \in \mathbb{R}^{3} ; \\
\text { and } f \in C^{2}(\mathbb{R}) \text { satisfying } \\
f(s) s \geq-K_{1}, \quad f^{\prime}(s) \geq-K_{2}, \quad\left|f^{\prime \prime}(s)\right| \leq K_{3}(1+|s|), \quad f(0)=0, \quad s \in \mathbb{R},
\end{array}\right.
$$

2 Here we adopt the common clash of notation for $(\cdot, \cdot)$ to mean both an inner product and represent a pair in a product space. It will be clear from the context which meaning is appropriate. 
for some positive constants $v, K_{i}$.

Remark 1.1. We note that above assumptions on $f$ imply the following bounds which are important in obtaining dissipative estimates.

a. There exists $K_{4}>0$ and $K_{5}>0$ such that $\left|f^{\prime}(s)\right| \leq K_{4}\left(1+|s|^{2}\right), \quad|f(s)| \leq K_{5}\left(1+|s|^{3}\right), \quad s \in \mathbb{R}$.

b. The anti-derivative ${ }^{3} F(s)=\int_{0}^{s} f(\tau) d \tau$ satisfies $-\frac{K_{2}}{2} s^{2} \leq F(s) \leq f(s) s+\frac{K_{2}}{2} s^{2}, \quad s \in \mathbb{R}$.

c. For all $\mu>0$ there exists $K_{\mu}>0$ such that $F(s) \geq-K_{\mu}-\mu s^{2}, \quad s \in \mathbb{R}$.

Also note that the assumption $f(0)=0$ is, in fact, not a restriction since $f(0)$ always can be included into the forcing term $g$.

We begin with some basic existence, continuity and dissipative estimate results. Particular attention is paid to the dependence of these results on the matrix $a$, assuming that the other variables ( $\Omega$ and $f$ ) are fixed. As these results are standard we shall omit the proofs, commenting here that they are easily argued by the techniques employed in Appendix A.

Theorem 1.1. Assume (H1). Then, for any initial data $\xi \in \mathcal{E}$, problem (1.2) possesses a unique energy solution $u$ with $\xi_{u} \in C\left(\mathbb{R}_{+} ; \varepsilon\right)$. Moreover, the following dissipative estimate is valid:

$$
\left\|\xi_{u}(t)\right\|_{\mathcal{E}}^{2}+\int_{t}^{\infty}\left\|\partial_{t} u(\tau)\right\|^{2} d \tau \leq M\left(\|\xi\|_{\mathcal{E}}\right) e^{-\beta t}+M(\|g\|), \quad t \geq 0,
$$

for some non-decreasing function $M$ and constant $\beta>0$ that depend only on $v$.

A consequence of the dissipative estimate (1.3), growth restrictions on $f$, and uniform ellipticity of $a(\cdot)$ we have the following continuous dependence on initial data.

Corollary 1.1. Let $u_{1}$ and $u_{2}$ be two energy solutions to problem (1.2) with initial data $\xi_{1}, \xi_{2} \in \mathcal{E}$ respectively. Then the following estimate

$$
\left\|\xi_{u_{1}}(t)-\xi_{u_{2}}(t)\right\|_{\mathcal{E}} \leq M e^{K t}\left\|\xi_{1}-\xi_{2}\right\|_{\mathcal{E}}, \quad t \geq 0,
$$

holds for some constant $M>0$ and $K=K\left(\left\|\xi_{1}\right\|_{\mathcal{E}},\left\|\xi_{2}\right\|_{\mathcal{E}},\|g\|, v\right)$.

Additionally, we have the following continuous dependence in $\mathcal{E}^{-1}$.

Corollary 1.2. Let $u_{1}$ and $u_{2}$ be two energy solutions to problem (1.2) with initial data $\xi_{1}, \xi_{2} \in \mathcal{E}$ respectively. Then the following estimate

$$
\left\|\xi_{u_{1}}(t)-\xi_{u_{2}}(t)\right\|_{\mathcal{E}^{-1}} \leq M e^{K t}\left\|\xi_{1}-\xi_{2}\right\|_{\mathcal{E}^{-1}}, \quad t \geq 0,
$$

holds for some constant $M>0$ and $K=K\left(\left\|\xi_{1}\right\|_{\mathcal{E}},\left\|\xi_{2}\right\|_{\mathcal{E}},\|g\|, v\right)$.

We now proceed to study the long-time behaviour of solutions $u$ from the point of view of infinite-dimensional dynamical systems. In particular the problem (1.2) defines a dynamical system $(\mathcal{E}, S(t))$ by

$$
S(t): \mathcal{E} \rightarrow \mathcal{E}, \quad S(t) \xi=\xi_{u}(t),
$$

where $u$ is a solution to the problem (1.2) with initial data $\xi$. The limit behaviour of a dissipative dynamical system as time goes to $+\infty$ can be described in terms of a so-called global attractor. Let us briefly recall its definition (see $[2,3,5,7])$.

Definition 1.1. Let $S(t): \mathcal{E} \rightarrow \mathcal{E}$ be a semi-group acting on a Banach space $\mathcal{E}$. Then a set $\mathcal{A}$ is called a global attractor for the dynamical system $(\mathcal{E}, S(t))$ if it possesses the following properties:

3 The upper-bound follows from noting that $g(s)=\int_{0}^{s} f(r) d r-f(s) s-\frac{K_{2}}{2} s^{2}$ attains its maximum at $s=0$. 
1. The set $\mathcal{A}$ is compact in $\mathcal{E}$;

2. The set $\mathcal{A}$ is strictly invariant:

$$
S(t) \mathcal{A}=\mathcal{A}, \quad \forall t \geq 0 ;
$$

3. The set $\mathcal{A}$ uniformly attracts every bounded set $B$ of $\mathcal{E}$, that is

$$
\lim _{t \rightarrow+\infty} \operatorname{dist}_{\mathcal{E}}(S(t) B, \mathcal{A})=0 .
$$

One can show that if a global attractor exists then it is unique. Also, the following description of the global attractor in terms of bounded trajectories is known (see e. g. [2, 3]):

$$
\mathcal{A}=\left\{\xi_{0} \in \mathcal{E}: \exists \xi(t) \in L^{\infty}(\mathbb{R} ; \mathcal{E}), \xi(0)=\xi_{0}, S(t) \xi(s)=\xi(t+s), s \in \mathbb{R}, t \geq 0\right\} .
$$

Now, the dissipative estimate (1.3) implies the existence of a bounded positively invariant absorbing set $\mathcal{B} \subset \mathcal{E}$ (which depends only on $v$ ):

$$
S(t) \mathcal{B} \subset \mathcal{B}, \quad \forall t \geq 0 .
$$

To prove that a global attractor exists for problem (1.2) we utilise the following classical result $([2,3,5,7])$.

Theorem 1.2. A dynamical system $(\mathcal{E}, S(t))$ possesses a global attractor $\mathcal{A}$ in $\mathcal{E}$ if the following conditions hold: 1. The dynamical system $(\mathcal{E}, S(t))$ is asymptotically compact: there exists a compact set $\mathscr{K} \subset \mathcal{E}$ such that

$$
\lim _{t \rightarrow+\infty} \operatorname{dist}_{\mathcal{E}}(S(t) B, \mathscr{K})=0, \quad \text { for all bounded sets } B \subset \mathcal{E} ;
$$

2. For each $t \geq 0$ the operators $S(t): \mathcal{E} \rightarrow \mathcal{E}$ are continuous.

Under such conditions, it follows that $\mathcal{A}$ not only exists but also $\mathcal{A} \subset \mathscr{K}$.

Note that Corollary 1.1 implies that the evolution operator $S(t)$, given by (1.4), has continuous dependence on the initial data. Let us focus on the existence of a compact attracting set.

Introducing the space

$$
\left\{\begin{array}{l}
\mathcal{E}^{1}:=\left\{\xi=\left(\xi^{1}, \xi^{2}\right) \in \mathcal{E} \mid \operatorname{div}\left(a \nabla \xi^{1}\right) \in L^{2}(\Omega), \xi^{2} \in H_{0}^{1}(\Omega)\right\}, \\
\|\xi\|_{\mathcal{E}^{1}}^{2}:=\left\|\operatorname{div}\left(a \nabla \xi^{1}\right)\right\|^{2}+\left\|\nabla \xi^{2}\right\|^{2},
\end{array}\right.
$$

we have the following known result that states there exists an attracting ball in $\mathcal{E}^{1}$.

Theorem 1.3. Assume (H1), and let $S(t)$ be the semi-group defined by (1.4). Then, there exists a ball in $\mathcal{E}^{1}$ that attracts the set $\mathcal{B}$, from (1.6), in $\mathcal{E}$. More precisely, the inequality

$$
\operatorname{dist}_{\mathcal{E}}\left(S(t) \mathcal{B}, B_{\mathcal{E}^{1}}(0, R)\right) \leq M e^{-\beta t}, \quad t \geq 0,
$$

holds for some positive constants $R, M$ and $\beta$ that depend only on $v$.

The proof of Theorem 1.3 is presented for the reader's convenience in Appendix A and is based on a splitting of trajectory $u$, into the smooth and contractive parts, that was developed in [31].

Consequently, as $\mathcal{E}^{1}$ is compact in $\mathcal{E}$ we see from Theorem 1.3 that $\mathscr{K}=B_{\mathcal{E}^{1}}(0, R)$ is a compact attracting set and, by Theorem 1.2, there exists a global attractor. That is, the following result holds.

Theorem 1.4. Assume (H1). Then, the dynamical system $(\mathcal{E}, S(t))$ given by (1.4) possesses a global attractor $\mathcal{A} \subset \mathcal{E}^{1}$ such that:

$$
\|\mathcal{A}\|_{\mathcal{E}^{1}} \leq M(\|g\|), \quad \mathcal{A}=\left.\mathcal{K}\right|_{t=0},
$$

where $\mathcal{K}$ is the set of bounded energy solutions to problem (1.2) defined for all $t \in \mathbb{R}$, cf. (1.5). 


\section{Smoothness of the global attractor}

Above we demonstrated that the global attractor $\mathcal{A}$ is a bounded subset of $\mathcal{E}^{1}$. We shall now establish some additional regularity of $\mathcal{A}$. These results will be used later on to derive homogenisation error estimates.

We are going to show that $\mathcal{A}$ is contained in the more regular set

$$
\left\{\begin{array}{l}
\mathcal{E}^{2}:=\left\{\xi \in \mathcal{E}^{1} \mid\left(\operatorname{div}\left(a \nabla \xi^{1}\right)+g\right) \in H_{0}^{1}(\Omega) \text { and } \operatorname{div}\left(a \nabla \xi^{2}\right) \in L^{2}(\Omega)\right\}, \\
\|\xi\|_{\mathcal{E}^{2}}^{2}:=\left\|\operatorname{div}\left(a \nabla \xi^{1}\right)+g\right\|_{H_{0}^{1}(\Omega)}^{2}+\left\|\operatorname{div}\left(a \nabla \xi^{1}\right)\right\|^{2}+\left\|\operatorname{div}\left(a \nabla \xi^{2}\right)\right\|^{2},
\end{array}\right.
$$

and that $\mathcal{A}$ is bounded in the following sense: $\|\mathcal{A}\|_{\mathcal{E}^{2}} \leq M$.

To this end, we shall show that $B_{\mathcal{E}^{1}}(0, R)$ is exponentially attracted, in $\mathcal{E}$, to some 'ball' ${ }^{4}$

$$
B_{\mathcal{E}^{2}}\left(0, R_{1}\right):=\left\{\xi \in \mathcal{E}^{2} \mid\|\xi\|_{\mathcal{E}^{2}} \leq R_{1}\right\} .
$$

Then by utilising the so-called transitivity property of exponential attraction we establish that $\mathcal{B}$ (from (1.6)) is attracted to $B_{\mathcal{E}^{2}}\left(0, R_{1}\right)$ exponentially in $\mathcal{E}$ and, therefore, we will show that $\mathcal{A}$ is bounded in $\mathcal{E}^{2}$.

Let us begin with the following theorem which provides a useful dissipative estimate for problem $(0.2)$ with initial data in $\mathcal{E}^{1}$ (see (1.7)).

Theorem 2.1. Assume (H1). Then for any initial data $\xi \in \mathcal{E}^{1}$ the energy solution $u$ to problem (1.2) is such that $\xi_{u} \in L^{\infty}\left(\mathbb{R}_{+} ; \mathcal{E}^{1}\right)$, and the following dissipative estimate is valid:

$$
\left\|\partial_{t}^{2} u(t)\right\|+\left\|\xi_{u}(t)\right\|_{\mathcal{E}^{1}} \leq M\left(\|\xi\|_{\mathcal{E}^{1}}\right) e^{-\beta t}+M(\|g\|), t \geq 0,
$$

for some non-decreasing function $M$ and constant $\beta>0$ that depend only on $v$.

Since this result is standard we omit the proof. We only remark here that, by differentiating the first equation of (1.2) in time, one first obtains a dissipative estimate for $\left\|\xi_{\partial_{t} u}(t)\right\|_{\mathcal{E}}$ which readily implies the uniform bound on $\|\operatorname{div}(a \nabla u)(t)\|$.

Remark 2.1. Note that by elliptic regularity we have the inequality

$$
\|u\|_{C^{\alpha}(\bar{\Omega})} \leq C\|\operatorname{div}(a \nabla u)\|, \quad C=C(v)>0,
$$

for sufficiently small $\alpha=\alpha(v)$ and admissible $u$. Here $C^{\alpha}(\bar{\Omega})$ is the Hölder space of order $\alpha$ :

$$
C^{\alpha}(\bar{\Omega})=\left\{u \in C(\bar{\Omega}): \sup _{\substack{x, y \in \bar{\Omega}, x \neq y}} \frac{|u(x)-u(y)|}{|x-y|^{\alpha}}<\infty\right\}, \quad\|u\|_{C^{\alpha}(\bar{\Omega})}:=\max _{x \in \bar{\Omega}}|u(x)|+\sup _{\substack{x, y \in \bar{\Omega}, x \neq y}} \frac{|u(x)-u(y)|}{|x-y|^{\alpha}} .
$$

Thus, we have a dissipative estimate for $u$, given by Theorem 2.1, in the $C^{\alpha}(\bar{\Omega})$ norm.

Consider $G \in H_{0}^{1}(\Omega)$ such that $-\operatorname{div}(a \nabla G)=g \in L^{2}(\Omega)$, and, for initial data $\xi \in B_{\mathcal{E}^{1}}(0, R)$, the decomposition of the solution $u$ to (1.2) as follows: $u=v+w$ where

$$
\left\{\begin{array}{l}
\partial_{t}^{2} v+y \partial_{t} v-\operatorname{div}(a \nabla v)=0, \quad x \in \Omega, t \geq 0 \\
\left.\xi_{v}\right|_{t=0}=\left(\xi^{1}-G, \xi^{2}\right),\left.\quad v\right|_{\partial \Omega}=0
\end{array}\right.
$$

and

$$
\left\{\begin{array}{l}
\partial_{t}^{2} w+y \partial_{t} w-\operatorname{div}(a \nabla w)=-f(u)+g, \quad x \in \Omega, t \geq 0, \\
\left.\xi_{w}\right|_{t=0}=(G, 0),\left.\quad w\right|_{\partial \Omega}=0
\end{array}\right.
$$

4 Note that the convex functional $\|\cdot\|_{\mathcal{E}^{2}}$ is not a norm and the set $\mathcal{E}^{2}$ is an affine subset of $\mathcal{\varepsilon}^{1}$. 
It is clear from standard linear estimates (e.g. Theorem 1.1 for $f=g=0$ ) that

$$
\left\|\xi_{v}(t)\right\|_{\mathcal{E}} \leq e^{-\beta t} M(\|g\|), \quad t \geq 0,
$$

for some constant $\beta>0$ and non-decreasing function $M$ that depend only on $v$. Additionally, we have the following lemma on the regularity of $w$.

Lemma 2.1. Assume (H1), $\xi \in B_{\mathcal{E}^{1}}(0, R)$ and $w$ solves (2.3). Then

$$
\|\operatorname{div}(a \nabla w)(t)+g\|_{H_{0}^{1}(\Omega)}+\left\|\operatorname{div}\left(a \nabla \partial_{t} w\right)(t)\right\| \leq M(\|g\|), \quad t \geq 0,
$$

for some non-decreasing function $M$ that depends only on $v$.

Proof. By differentiating the first equation of (2.3) in time and by our choice of initial data $(G, 0)$ we find that $p:=\partial_{t} w$ solves

$$
\left\{\begin{array}{l}
\partial_{t}^{2} p+y \partial_{t} p-\operatorname{div}(a \nabla p)=-f^{\prime}(u) \partial_{t} u=: G_{1}, \quad x \in \Omega, t \geq 0, \\
\left.\xi_{p}\right|_{t=0}=\left(0,-f\left(\xi^{1}\right)\right),\left.\quad p\right|_{\partial \Omega}=0 .
\end{array}\right.
$$

Moreover, $q:=\partial_{t} p$ solves

$$
\left\{\begin{array}{l}
\partial_{t}^{2} q+y \partial_{t} q-\operatorname{div}(a \nabla q)=-f^{\prime \prime}(u)\left|\partial_{t} u\right|^{2}-f^{\prime}(u) \partial_{t}^{2} u=: G_{2}, \quad x \in \Omega, t \geq 0, \\
\left.\xi_{q}\right|_{t=0}=\left(-f\left(\xi^{1}\right), y f\left(\xi^{1}\right)-f^{\prime}\left(\xi^{1}\right) \xi^{2}\right),\left.\quad q\right|_{\partial \Omega}=0 .
\end{array}\right.
$$

By the dissipative estimate in $\mathcal{E}^{1}$ (cf. Theorem 2.1 and Remark 2.1) we find that

$$
\left\|\nabla \partial_{t} u(t)\right\|+\|u(t)\|_{C^{\alpha}(\bar{\Omega})} \leq M(\|g\|), \quad t \geq 0 .
$$

This inequality and the conditions on the non-linearity $f$ (see (H1)) imply that

$$
\begin{aligned}
& \left\|\xi_{p}(0)\right\|_{\mathcal{E}}+\left\|G_{1}\right\|_{L^{\infty}\left(\mathbb{R}_{+} ; L^{2}(\Omega)\right)} \leq M(\|g\|) ; \\
& \left\|\xi_{q}(0)\right\|_{\mathcal{E}}+\left\|G_{2}\right\|_{L^{\infty}\left(\mathbb{R}_{+} ; L^{2}(\Omega)\right)} \leq M(\|g\|) .
\end{aligned}
$$

Therefore, using the dissipative estimate in $\mathcal{E}((1.3))$ we conclude

$$
\|\nabla p(t)\|+\left\|\partial_{t} p(t)\right\| \leq M(\|g\|), \quad \& \quad\|\nabla q(t)\|+\left\|\partial_{t} q(t)\right\| \leq M(\|g\|), \quad t \geq 0 .
$$

Returning back to $p=\partial_{t} w$, we rewrite (2.5) to find

$$
\left\|\operatorname{div}\left(a \nabla \partial_{t} w\right)(t)\right\|=\left\|-G_{1}(t)+y \partial_{t} p(t)+\partial_{t} q(t)\right\| \leq M(\|g\|), \quad t \geq 0 .
$$

Rewriting the first equation in (2.3), and using cubic growth of $f$ (see Remark 1.1.a) gives

$$
\|\operatorname{div}(a \nabla w)(t)+g\|_{H_{0}^{1}(\Omega)}=\|q(t)+y p(t)+f(u(t))\|_{H_{0}^{1}(\Omega)} \leq M(\|g\|), \quad t \geq 0 .
$$

Hence, the desired result holds and the proof is complete.

Combining (2.4) and Lemma 2.1 produces the following result.

Corollary 2.1. Assume (H1) and let $S(t)$ be the semi-group defined by (1.4). Then, there exists a 'ball' in $\mathcal{E}^{2}$ that attracts $B_{\mathcal{E}^{1}}(0, R)$ in $\mathcal{E}$. More precisely, the inequality

$$
\operatorname{dist}_{\mathcal{E}}\left(S(t) B_{\mathcal{E}^{1}}(0, R), B_{\mathcal{E}^{2}}\left(0, R_{1}\right)\right) \leq M e^{-\beta t}, \quad t \geq 0,
$$

holds for some positive constants $R_{1}, M$ and $\beta$ that depend only on $v$.

Let us now recall the so-called transitivity property of exponential attraction (cf. [30, Theorem 5.1] for a proof): 
Theorem 2.2. Let $E$ be a Banach space, $S(t)$ a semi-group acting on $E$, and $E_{1}$ be a positively invariant subset of $E$, i.e. $S(t) E_{1} \subset E_{1}$ for all $t \geq 0$, such that

$$
\left\|S(t) \xi^{1}-S(t) \xi^{2}\right\|_{E} \leq M_{0} e^{K_{0} t}\left\|\xi^{1}-\xi^{2}\right\|_{E}, \quad \xi^{1}, \xi^{2} \in E_{1},
$$

for some constants $M_{0}, K_{0}>0$. Furthermore, assume that there exist subsets $E_{2} \subset E_{1}$ and $E_{3} \subset E$ such that

$$
\operatorname{dist}_{E}\left(S(t) E_{1}, E_{2}\right) \leq M_{1} e^{-\beta_{1} t}, \quad \operatorname{dist}_{E}\left(S(t) E_{2}, E_{3}\right) \leq M_{2} e^{-\beta_{2} t}, \quad t \geq 0,
$$

for some $M_{1}, M_{2}, \beta_{1}>0$ and $\beta_{2}>0$. Then

$$
\operatorname{dist}_{E}\left(S(t) E_{1}, E_{3}\right) \leq M e^{-\beta t}, \quad t \geq 0,
$$

for $M=M_{0} M_{1}+M_{2}$ and $\beta=\frac{\beta_{1} \beta_{2}}{K_{0}+\beta_{1}+\beta_{2}}$.

Note that Theorem 1.1 (in particular (1.6)), Theorem 1.3 and Corollary 2.1 imply that the assumptions of the above theorem hold for $E=\mathcal{E}, E_{1}=\mathcal{B}, E_{2}=B_{\mathcal{E}^{1}}(0, R)$ and $E_{3}=B_{\mathcal{E}^{2}}\left(0, R_{1}\right)$. Therefore, we see that $B_{\mathcal{E}^{2}}\left(0, R_{1}\right)$ attracts the positively invariant absorbing set $\mathcal{B}$ and, therefore, bounded sets in $\mathcal{E}$. That is the following result holds.

Theorem 2.3. Assume (H1), $S(t)$ given by (1.4) and $B_{\mathcal{E}^{2}}\left(0, R_{1}\right)$ given by Corollary 2.1. Then, for every bounded $B$ in $\mathcal{E}$ the following assertion

$$
\operatorname{dist}_{\mathcal{E}}\left(S(t) B, B_{\mathcal{E}^{2}}\left(0, R_{1}\right)\right) \leq M\left(\|B\|_{\mathcal{E}}\right) e^{-\beta t}, \quad t \geq 0,
$$

holds for some non-decreasing $M$ and $\beta>0$ that depend only on $v$.

We are now ready to prove that the global attractor is bounded in $\varepsilon^{2}$.

Theorem 2.4. Assume (H1) and let $\mathcal{A}$ be the global attractor of the dynamical system $(\mathcal{E}, S(t))$ given by (1.4). Then

$$
\|\mathcal{A}\|_{\mathcal{E}^{2}} \leq M(\|g\|),
$$

for some non-decreasing $M$ that depends only on $v$.

Remark 2.2. Note that (2.6) implies the following estimate

$$
\|\mathcal{A}\|_{\left(C^{\alpha}(\bar{\Omega})\right)^{2}} \leq M(\|g\|),
$$

for a non-decreasing function $M$ that depends only on $v$ and the exponent $\alpha$ from Remark 2.1.

Proof of Thoerem 2.4. The proof follows from the strict invariance of the global attractor (property 2. of Definition 1.1) and Theorem 2.3. Indeed, for an arbitrary $\delta$-neighbourhood $\mathcal{O}_{\delta}\left(B_{\mathcal{E}^{2}}\left(0, R_{1}\right)\right)$ of $B_{\mathcal{E}^{2}}\left(0, R_{1}\right)$ in $\mathcal{E}$, one has

$$
\mathcal{A}=S(t) \mathcal{A} \subset \mathcal{O}_{\delta}\left(B_{\mathcal{E}^{2}}\left(0, R_{1}\right)\right),
$$

for some $t=t(\delta)$. Therefore $\mathcal{A} \subset\left[B_{\mathcal{E}^{2}}\left(0, R_{1}\right)\right]_{\mathcal{E}}$ and it remains to note that, since $B_{\mathcal{E}^{2}}(0, R)$ is closed in $\mathcal{E}$, the identity $\left[B_{\mathcal{E}^{2}}\left(0, R_{1}\right)\right]_{\mathcal{E}}=B_{\mathcal{E}^{2}}\left(0, R_{1}\right)$ holds.

We end this section with one more result which will be useful later.

Theorem 2.5. Assume (H1). Then, for any initial data $\xi \in \mathcal{E}^{2}$, the energy solution u to problem (1.2) is such that $\xi_{u} \in L^{\infty}\left(\mathbb{R}_{+} ; \varepsilon^{2}\right)$ and the following dissipative estimate is valid:

$$
\left\|\partial_{t}^{3} u(t)\right\|+\left\|\nabla \partial_{t}^{2} u(t)\right\|+\left\|\xi_{u}(t)\right\|_{\mathcal{E}^{2}} \leq M\left(\|\xi\|_{\mathcal{E}^{2}}\right) e^{-\beta t}+M(\|g\|), \quad t \geq 0,
$$

for some non-decreasing function $M$ and constant $\beta>0$ that depend only on $v>0$. 
The proof is very close to the proof of Lemma 2.1 and for this reason is omitted. We only remark that, since $\varepsilon^{2} \subset \mathcal{E}^{1}$ and the dissipative estimate in $\mathcal{E}^{1}$ is already known, we see that the quantity $\|u(t)\|_{L^{\infty}(\Omega)}$ is bounded. Thus, basically, one applies linear dissipative estimates to the equations for $p$ and $q$ in the proof of Lemma 2.1 with the appropriately changed initial data.

\section{Homogenisation and convergence of global attractors}

Let us now consider the dynamical systems $S_{\varepsilon}(t)$ and $S_{0}(t)$ generated by problems $(0.1)$ and (0.2) respectively. In Theorem 2.4 we established that $S_{\varepsilon}$ (respect. $S_{0}$ ) has a global attractor $\mathcal{A}^{\varepsilon}$ (respect. $\mathcal{A}^{0}$ ). Moreover, Theorem 2.4 informs us that $\mathcal{A}^{\varepsilon}$ is a, uniformly in $\varepsilon$, bounded subset of $\varepsilon_{\varepsilon}^{2}$ and $\mathcal{A}^{0}$ is a bounded subset of $\varepsilon_{0}^{2}$, where

$$
\left\{\begin{array}{l}
\varepsilon_{\varepsilon}^{2}:=\left\{\xi \in\left(H_{0}^{1}(\Omega)\right)^{2} \mid\left(\operatorname{div}\left(a\left(\frac{\dot{\bar{\varepsilon}}}{\bar{\varepsilon}}\right) \nabla \xi^{1}\right)+g\right) \in H_{0}^{1}(\Omega), \operatorname{div}\left(a(\dot{\bar{\varepsilon}}) \nabla \xi^{2}\right) \in L^{2}(\Omega)\right\}, \\
\|\xi\|_{\mathcal{E}_{\varepsilon}^{2}}^{2}:=\left\|\operatorname{div}\left(a\left(\frac{\dot{\bar{\varepsilon}}}{\bar{\varepsilon}}\right) \nabla \xi^{1}\right)+g\right\|_{H_{0}^{1}(\Omega)}^{2}+\left\|\operatorname{div}\left(a(\dot{\bar{\varepsilon}}) \nabla \xi^{1}\right)\right\|^{2}+\left\|\operatorname{div}\left(a\left(\frac{\dot{\bar{\varepsilon}}}{\bar{\varepsilon}}\right) \nabla \xi^{2}\right)\right\|^{2},
\end{array}\right.
$$

and

$$
\left\{\begin{array}{l}
\varepsilon_{0}^{2}:=\left\{\xi \in\left(H_{0}^{1}(\Omega)\right)^{2} \mid\left(\operatorname{div}\left(a^{h} \nabla \xi^{1}\right)+g\right) \in H_{0}^{1}(\Omega), \operatorname{div}\left(a^{h} \nabla \xi^{2}\right) \in L^{2}(\Omega)\right\}, \\
\|\xi\|_{\mathcal{E}_{0}^{2}}^{2}:=\left\|\operatorname{div}\left(a^{h} \nabla \xi^{1}\right)+g\right\|_{H_{0}^{1}(\Omega)}^{2}+\left\|\operatorname{div}\left(a^{h} \nabla \xi^{1}\right)\right\|^{2}+\left\|\operatorname{div}\left(a^{h} \nabla \xi^{2}\right)\right\|^{2} .
\end{array}\right.
$$

Remark 3.1. We note that, by elliptic regularity (see Remark 2.1), the global attractors $\mathcal{A}^{\varepsilon}$ are uniformly in $\varepsilon$ bounded subsets of $\mathcal{E}_{\varepsilon}^{2} \cap\left(C^{\alpha}(\bar{\Omega})\right)^{2}$. Additionally for $\mathcal{A}^{0}$, as $a^{h}$ is constant, we can readily deduce that $\mathcal{A}^{0}$ is a bounded subset of $\varepsilon_{0}^{2} \cap\left(H^{2}(\Omega)\right)^{2}$. That is, the inequalities

$$
\left\|\mathcal{A}^{\varepsilon}\right\|_{\mathcal{E}_{\varepsilon}^{2}}+\left\|\mathcal{A}^{\varepsilon}\right\|_{\left(C^{\alpha}(\bar{\Omega})\right)^{2}} \leq M(\|g\|), \quad \& \quad\left\|\mathcal{A}^{0}\right\|_{\mathcal{E}_{0}^{2}}+\left\|\mathcal{A}^{0}\right\|_{\left(H^{2}(\Omega)\right)^{2}} \leq M(\|g\|),
$$

hold for some non-decreasing function $M$ independent of $\varepsilon$.

The main result of this section is the following theorem which establishes convergence of the global attractors $\mathcal{A}^{\varepsilon}$ to the global attractor $\mathcal{A}^{0}$ in the one-sided Hausdorff distance.

Theorem 3.1. The global attractor $\mathcal{A}^{\varepsilon}$ of the problem (0.1) converges to the global attractor $\mathcal{A}^{0}$ of the homogenised problem (0.2) in the following sense

$$
\lim _{\varepsilon \rightarrow 0} \operatorname{dist}_{\left(C^{\beta}(\bar{\Omega})\right)^{2}}\left(\mathcal{A}^{\varepsilon}, \mathcal{A}^{0}\right)=0,
$$

for any $0 \leq \beta<\alpha$ where $\alpha$ is given in Remark 3.1.

To prove Theorem 3.1 we shall use the following classical homogenisation theorem for elliptic PDEs (see for example [15, Section 1]).

Theorem 3.2. (Homogenisation theorem) Let $\Omega \subset \mathbb{R}^{3}$ be a bounded smooth domain, a $(\cdot)$ a positive bounded periodic matrix and $\varepsilon_{n} \rightarrow 0$ as $n \rightarrow \infty$. Then for any sequence $g_{n} \in H^{-1}(\Omega)$ that strongly converges to $g$ in $H^{-1}(\Omega)$ we have that $u_{n} \in H_{0}^{1}(\Omega)$ the weak solution of

$$
\operatorname{div}\left(a\left(\frac{x}{\varepsilon_{n}}\right) \nabla u_{n}\right)=g_{n},
$$

weakly converges in $H_{0}^{1}(\Omega)$ to $u_{0}$ the weak solution of

$$
\operatorname{div}\left(a^{h} \nabla u_{0}\right)=g .
$$

Remark 3.2. In general, one cannot expect strong convergence of $u_{n}$ to $u_{0}$ in $H_{0}^{1}(\Omega)$ since this would imply that the homogenised matrix $a^{h}$ is simply the average $\int_{Q} a(y) d y$. Clearly this formula for the homogenised matrix is, in general, not true and it is known that the equality $a^{h}=\int_{Q} a(y) d y$ holds if, and only if, $\operatorname{div}_{y} a=0$ in weak sense. 
A consequence of the above observation is that, in general, we can not expect convergence of the attractors $\mathcal{A}^{\varepsilon}$ to $\mathcal{A}^{0}$ in the strong topology of $\mathcal{E}$. To obtain such convergence results a correction to $\mathcal{A}^{0}$ needs to be made, see Section 5 for further information.

Proof of Theorem 3.1. Fix an arbitrary sequence $\varepsilon_{n} \rightarrow 0$ and $\xi_{n} \in \mathcal{A}^{\varepsilon_{n}}$. To prove the result it is sufficient to show that there exists $\xi_{0} \in \mathcal{A}^{0}$ such that $\xi_{n}$ converges, up to some subsequence, to $\xi_{0}$ in $\left(C^{\beta}(\bar{\Omega})\right)^{2}$ as $n \rightarrow \infty$.

For each $n \in \mathbb{N}$, we denote by $u_{n} \in \mathcal{K}^{\varepsilon_{n}}$ the bounded (for all time) in $\mathcal{E}$ solution of (0.1) that satisfies $\xi_{u_{n}}(0)=\xi_{n}$. Now, $\mathcal{A}^{\varepsilon}$ is a (uniformly in $\varepsilon$ ) bounded subset of $\left(H_{0}^{1}(\Omega) \cap C^{\alpha}(\bar{\Omega})\right)^{2}$ (see Remark 3.1). Moreover, it is well-known that $C^{\alpha}(\bar{\Omega})$ is compactly embedded in $C^{\beta}(\bar{\Omega})$, for any $0 \leq \beta<\alpha$. Therefore, up to some discarded subsequence,

$$
\xi_{n} \text { converges strongly in }\left(C^{\beta}(\bar{\Omega})\right)^{2} \text { to some } \xi_{0} \in\left(H_{0}^{1}(\Omega) \cap C^{\beta}(\bar{\Omega})\right)^{2} .
$$

It remains to prove that $\xi_{0} \in \mathcal{A}^{0}$, and this is established if we demonstrate that $\xi_{0}=\xi_{u_{0}}(0)$ for some bounded (for all time) in $\mathcal{E}$ solution $u_{0}$ to (0.2). The remainder of the proof is to establish the existence of such a $u_{0}$. In what follows convergence is meant up to an appropriately discarded subsequence.

By Remark 3.1 and the strict invariance of $\mathcal{A}^{\varepsilon}$ (property 2 of Definition (1.1)) there exists $M>0$ such that

$$
\begin{aligned}
\left\|\nabla u_{n}(t)\right\|+\left\|\operatorname{div}\left(a\left(\frac{x}{\varepsilon_{n}}\right) \nabla u_{n}\right)(t)\right\|+\left\|u_{n}(t)\right\|_{C^{\alpha}(\bar{\Omega})} \\
+\left\|\nabla \partial_{t} u_{n}(t)\right\|+\left\|\operatorname{div}\left(a\left(\frac{x}{\varepsilon_{n}}\right) \nabla \partial_{t} u_{n}\right)(t)\right\|+\left\|\partial_{t} u_{n}(t)\right\|_{C^{\alpha}(\bar{\Omega})} \leq M,
\end{aligned}
$$

for all $n \in \mathbb{N}$ and all $t \in \mathbb{R}$.

Let us fix $z \in \mathbb{Z}$. Using (3.4) we find

$$
u_{n} \text { is bounded in } W_{1}:=\left\{w \in L^{\infty}\left([z, z+2] ; H_{0}^{1}(\Omega)\right) \mid \partial_{t} w \in L^{\infty}\left([z, z+2] ; L^{2}(\Omega)\right)\right\} .
$$

Similarly, since (cf. (0.1))

$$
\partial_{t}^{2} u_{n}=-y \partial_{t} u_{n}+\operatorname{div}\left(a\left(\frac{x}{\varepsilon_{n}}\right) \nabla u_{n}\right)-f\left(u_{n}\right)+g,
$$

assertion (3.4) and the cubic growth condition of $f$ (Remark 1.1(a)) imply that

$$
\partial_{t} u_{n} \text { is bounded in } W_{1} .
$$

Furthermore, differentiating (3.5) in $t$ gives

$$
\partial_{t}^{3} u_{n}=-y \partial_{t}^{2} u_{n}+\operatorname{div}\left(a\left(\frac{x}{\varepsilon_{n}}\right) \nabla \partial_{t} u_{n}\right)-f^{\prime}\left(u_{n}\right) \partial_{t} u_{n} .
$$

This equation, along with (3.4), the boundedness of $\partial_{t} u_{n}$ in $W_{1}$ and growth assumption on $f$ imply that

$$
\partial_{t}^{2} u_{n} \text { is bounded in }\left\{w \in L^{\infty}\left([z, z+2] ; L_{2}(\Omega)\right) \mid \partial_{t} w \in L^{\infty}\left([z, z+2] ; H^{-1}(\Omega)\right)\right\} .
$$

Therefore, since the embeddings $H_{0}^{1}(\Omega) \subset L^{2}(\Omega)$ and $L^{2}(\Omega) \subset H^{-1}(\Omega)$ are compact, by Aubin-Lions lemma we deduce that

$$
\begin{array}{cl}
u_{n} \longrightarrow u & \text { strongly in } C\left([z, z+2] ; L^{2}(\Omega)\right) \text { as } n \longrightarrow \infty ; \\
\partial_{t} u_{n} \longrightarrow \partial_{t} u & \text { strongly in } C\left([z, z+2] ; L^{2}(\Omega)\right) \text { as } n \rightarrow \infty ; \\
\partial_{t}^{2} u_{n} \longrightarrow \partial_{t}^{2} u & \text { strongly in } C\left([z, z+2] ; H^{-1}(\Omega)\right) \text { as } n \rightarrow \infty .
\end{array}
$$

Let us demonstrate that $u$ solves (0.2) on the time interval $[z, z+2]$. To this end we are going to pass to the limit in

$$
-\operatorname{div}\left(a\left(\frac{x}{\varepsilon_{n}}\right) \nabla u_{n}\right)=-\partial_{t}^{2} u_{n}-y \partial_{t} u_{n}-f\left(u_{n}\right)+g=: h_{n} .
$$

Due to (3.6) we know that

$$
h_{n}(t) \longrightarrow-\partial_{t}^{2} u(t)-y \partial_{t} u(t)-f(u(t))+g \text { strongly in } H^{-1}(\Omega) \text { for all } t \in[z, z+2] .
$$


Therefore, by an application of the homogenisation theorem (Theorem 3.2), we conclude, that for every $t \in$ $[z, z+2], u_{n}(t)$ weakly converges in $H_{0}^{1}(\Omega)$ to the solution $u_{0}(t)$ of the homogenised problem

$$
-\operatorname{div}\left(a^{h} \nabla u_{0}(t)\right)=-\partial_{t}^{2} u(t)-y \partial_{t} u(t)-f(u(t))+g .
$$

It follows from (3.6) and the weak convergence $u_{n}(t) \rightarrow u_{0}(t)$ in $H_{0}^{1}(\Omega)$ that $u(t)=u_{0}(t)$ for all $t \in[z, z+2]$. Consequently, from this identity and the above equation, we see that $u_{0}$ (weakly) solves

$$
\partial_{t}^{2} u_{0}+y \partial_{t} u_{0}-\operatorname{div}\left(a^{h} \nabla u_{0}\right)+f\left(u_{0}\right)=g, \quad t \in[z, z+2] .
$$

Let us argue that the above equation holds for all time. Indeed, by a Cantor diagonalisation argument we see that the convergences (3.6) can be taken to hold for all $z \in \mathbb{Z}$. Then, by noting that any $\phi \in C_{0}^{\infty}\left(\mathbb{R} ; C_{0}^{\infty}(\Omega)\right.$ ) can be represented as a finite sum of smooth functions whose individual supports (w.r.t to time) are in some $[z, z+2]$, we deduce that $u_{0}$ weakly solves the homogenised equation (0.2). Hence, $u_{0}$ is a bounded in $\mathcal{E}$ solution to (0.2) for all time.

It remains to show that $\xi_{u_{0}}(0)=\left(u_{0}(0), \partial_{t} u_{0}(0)\right)$ equals $\xi_{0}$. On the one hand, from (3.3) we see that $\xi_{n}$ converges strongly to $\xi_{0}$ in $\left(L^{2}(\Omega)\right)^{2}$. On the other hand, by (3.6) (for $\left.z=0\right) \xi_{n}=\left(u_{n}(0), \partial_{t} u_{n}(0)\right)$ converges strongly to $\left(u_{0}(0), \partial_{t} u_{0}(0)\right)$ in $\left(L^{2}(\Omega)\right)^{2}$. Hence, $\left(u_{0}(0), \partial_{t} u_{0}(0)\right)=\xi_{0}$ and the proof is complete.

\section{Rate of convergence to the homogenised global attractor}

We shall begin with recalling an important result on error estimates in homogenisation theory of elliptic PDEs. Recall, for fixed $\varepsilon>0$, the mappings

$$
A_{\varepsilon} u:=-\operatorname{div}(a(\dot{\bar{\varepsilon}}) \nabla u), \quad \& \quad A_{0} u:=-\operatorname{div}\left(a^{h} \nabla u\right) .
$$

Theorem 4.1 (Theorem 3.1, [16]). Let $\Omega \subset \mathbb{R}^{3}$ be a bounded smooth domain, symmetric periodic matrix a $(\cdot)$ satisfying uniform ellipticity and boundedness assumptions, $A_{\varepsilon}$ and $A_{0}$ given by (4.1) and $g \in L^{2}(\Omega)$. Let also $u^{\varepsilon}, u^{0} \in H_{0}^{1}(\Omega)$ solve the problems

$$
\left\{\begin{array} { l } 
{ A _ { \varepsilon } u ^ { \varepsilon } = g , \quad \text { in } \Omega , } \\
{ u ^ { \varepsilon } | _ { \partial \Omega } = 0 , }
\end{array} \quad \& \quad \left\{\begin{array}{l}
A_{0} u^{0}=g, \quad \text { in } \Omega, \\
\left.u^{0}\right|_{\partial \Omega}=0 .
\end{array}\right.\right.
$$

Then, the following estimate

$$
\left\|u^{\varepsilon}-u^{0}\right\| \leq C \varepsilon\|g\|
$$

holds for some constant $C=C(v, \Omega)$.

Remark 4.1. Note that inequality (4.2) is equivalent to the following operator estimate on resolvents:

$$
\left\|A_{\varepsilon}^{-1}-A_{0}^{-1}\right\|_{\mathcal{L}\left(L^{2}(\Omega)\right)} \leq C \varepsilon .
$$

In what follows we wish to compare properties of the semi-groups associated to (0.1) and (0.2) via estimates in terms of $\varepsilon$. In fact, we shall provide stronger estimates in terms of the difference $\left\|A_{\varepsilon}^{-1}-A_{0}^{-1}\right\|_{\mathcal{L}\left(L^{2}(\Omega)\right)}$. The mentioned $\varepsilon$ estimates then immediately follow by Remark 4.1.

Our first important result is the following continuity estimate.

Theorem 4.2. Let $\mathcal{E}_{\varepsilon}^{2}$ be the set (3.1), $R>0$. Then, for all $\xi \in B_{\mathcal{E}_{\varepsilon}^{2}}(0, R)=\left\{\xi \in \mathcal{E}_{\varepsilon}^{2},\|\xi\|_{\mathcal{E}_{\varepsilon}^{2}} \leq R\right\}$, the inequality

$$
\left\|S_{\varepsilon}(t) \xi-S_{0}(t) \xi\right\|_{\mathcal{E}^{-1}} \leq M e^{K t}\left\|A_{\varepsilon}^{-1}-A_{0}^{-1}\right\|_{\mathcal{L}\left(L^{2}(\Omega)\right)}, \quad t \geq 0,
$$

holds for some non-decreasing functions $M=M(R,\|g\|)$ and $K=K(R,\|g\|)$ which are independent of $\varepsilon>0$. 
Proof of Theorem 4.2. Let us fix $\xi$, set $\xi_{u^{\varepsilon}}(t):=S_{\varepsilon}(t) \xi, \xi_{u^{0}}(t):=S_{0}(t) \xi$, and define $r^{\varepsilon}:=u^{\varepsilon}-u^{0}$. Then, $r^{\varepsilon}$ solves

$$
\left\{\begin{array}{l}
\partial_{t}^{2} r^{\varepsilon}+y \partial_{t} r^{\varepsilon}+A_{0} r^{\varepsilon}=A_{0} u^{\varepsilon}-A_{\varepsilon} u^{\varepsilon}+f\left(u^{0}\right)-f\left(u^{\varepsilon}\right), \quad x \in \Omega, t \geq 0 \\
\left.\xi_{r^{\varepsilon}}\right|_{t=0}=0,\left.\quad r^{\varepsilon}\right|_{\partial \Omega}=0 .
\end{array}\right.
$$

By testing the first equation in (4.4) with $A_{0}^{-1} \partial_{t} r^{\varepsilon}$ we deduce that

$$
\begin{array}{r}
\frac{d}{d t}\left(\frac{1}{2}\left(\partial_{t} r^{\varepsilon}, A_{0}^{-1} \partial_{t} r^{\varepsilon}\right)+\frac{1}{2}\left\|r^{\varepsilon}\right\|^{2}\right)+y\left(\partial_{t} r^{\varepsilon}, A_{0}^{-1} \partial_{t} r^{\varepsilon}\right)= \\
\left(A_{0} u^{\varepsilon}-A_{\varepsilon} u^{\varepsilon}, A_{0}^{-1} \partial_{t} r^{\varepsilon}\right)+\left(f\left(u^{0}\right)-f\left(u^{\varepsilon}\right), A_{0}^{-1} \partial_{t} r^{\varepsilon}\right) .
\end{array}
$$

We compute

$$
\begin{aligned}
\left(A_{0} u^{\varepsilon}-A_{\varepsilon} u^{\varepsilon}, A_{0}^{-1} \partial_{t} r^{\varepsilon}\right)=\left(A_{0} u^{\varepsilon}, A_{0}^{-1} \partial_{t} r^{\varepsilon}\right)-\left(A_{\varepsilon} u^{\varepsilon}, A_{0}^{-1} \partial_{t} r^{\varepsilon}\right) & =\left(u^{\varepsilon}, \partial_{t} r^{\varepsilon}\right)-\left(A_{\varepsilon} u^{\varepsilon}, A_{0}^{-1} \partial_{t} r^{\varepsilon}\right) \\
& =\left(A_{\varepsilon} u^{\varepsilon}, A_{\varepsilon}^{-1} \partial_{t} r^{\varepsilon}\right)-\left(A_{\varepsilon} u^{\varepsilon}, A_{0}^{-1} \partial_{t} r^{\varepsilon}\right) \\
& =\left(A_{\varepsilon} u^{\varepsilon},\left(A_{\varepsilon}^{-1}-A_{0}^{-1}\right) \partial_{t} r^{\varepsilon}\right) .
\end{aligned}
$$

Furthermore,

$$
\left(A_{\varepsilon} u^{\varepsilon},\left(A_{\varepsilon}^{-1}-A_{0}^{-1}\right) \partial_{t} r^{\varepsilon}\right)=\frac{d}{d t}\left(A_{\varepsilon} u^{\varepsilon},\left(A_{\varepsilon}^{-1}-A_{0}^{-1}\right) r^{\varepsilon}\right)-\left(A_{\varepsilon} \partial_{t} u^{\varepsilon},\left(A_{\varepsilon}^{-1}-A_{0}^{-1}\right) r^{\varepsilon}\right) .
$$

Therefore, we can rewrite (4.5) as

$$
\frac{d}{d t} \Lambda+y\left(\partial_{t} r^{\varepsilon}, A_{0}^{-1} \partial_{t} r^{\varepsilon}\right)=-\left(A_{\varepsilon} \partial_{t} u^{\varepsilon},\left(A_{\varepsilon}^{-1}-A_{0}^{-1}\right) r^{\varepsilon}\right)+\left(f\left(u^{0}\right)-f\left(u^{\varepsilon}\right), A_{0}^{-1} \partial_{t} r^{\varepsilon}\right),
$$

for

$$
\Lambda(t):=\frac{1}{2}\left(\partial_{t} r^{\varepsilon}(t), A_{0}^{-1} \partial_{t} r^{\varepsilon}(t)\right)+\frac{1}{2}\left\|r^{\varepsilon}(t)\right\|^{2}-\left(A_{\varepsilon} u^{\varepsilon}(t),\left(A_{\varepsilon}^{-1}-A_{0}^{-1}\right) r^{\varepsilon}(t)\right), \quad t \geq 0 .
$$

We now aim to bound the right-hand-side of (4.6) in terms of $\left\|A_{\varepsilon}^{-1}-A_{0}^{-1}\right\|_{\mathcal{L}\left(L^{2}(\Omega)\right)}^{2}$ and $\Lambda$, then subsequently apply Gronwall's inequality and the following standard estimate

$$
v\|\phi\|_{H^{-1}(\Omega)}^{2} \leq\left(\phi, A_{0}^{-1} \phi\right) \leq v^{-1}\|\phi\|_{H^{-1}(\Omega)}^{2}, \quad \phi \in H^{-1}(\Omega)
$$

to deduce the desired result.

To this end, let us first estimate the non-linear term. Using the growth restriction on $f^{\prime}$ (see Remark 1.1a) and Hölder's inequality (for exponents $\left.\left(p_{1}, p_{2}, p_{3}\right)=(3,2,6)\right)$ we compute

$$
\begin{aligned}
\left|\left(f\left(u^{\varepsilon}\right)-f\left(u^{0}\right), A_{0}^{-1} \partial_{t} r^{\varepsilon}\right)\right| & \leq M\left(\left(1+\left|u^{\varepsilon}\right|^{2}+\left|u^{0}\right|^{2}\right)\left|r^{\varepsilon}\right|,\left|A_{0}^{-1} \partial_{t} r^{\varepsilon}\right|\right) \\
& \leq M\left\|1+\left|u^{\varepsilon}\right|^{2}+\left|u^{0}\right|^{2}\right\|_{L^{3}(\Omega)}\left\|r^{\varepsilon}\right\|\left\|A_{0}^{-1} \partial_{t} r^{\varepsilon}\right\|_{L^{6}(\Omega)} .
\end{aligned}
$$

Then, by the Sobolev embedding $L^{6}(\Omega) \subset H^{1}(\Omega)$, the fact that $u^{\varepsilon}$ and $u^{0}$ are bounded in $\mathcal{E}$ (see dissipative estimate (1.3)) and (4.7) we compute

$$
\begin{aligned}
\left|\left(f\left(u^{\varepsilon}\right)-f\left(u^{0}\right), A_{0}^{-1} \partial_{t} r^{\varepsilon}\right)\right| \leq M\left\|r^{\varepsilon}\right\|\left\|A_{0}^{-1} \partial_{t} r^{\varepsilon}\right\|_{H^{1}(\Omega)} & \leq M\left\|r^{\varepsilon}\right\|\left\|\partial_{t} r^{\varepsilon}\right\|_{H^{-1}(\Omega)} \\
& \leq M\left\|r^{\varepsilon}\right\|\left(\partial_{t} r^{\varepsilon}, A_{0}^{-1} \partial_{t} r^{\varepsilon}\right)^{\frac{1}{2}} \\
& \leq M_{1}\left(\frac{1}{2}\left\|r^{\varepsilon}\right\|^{2}+\frac{1}{2}\left(\partial_{t} r^{\varepsilon}, A_{0}^{-1} \partial_{t} r^{\varepsilon}\right)\right),
\end{aligned}
$$

for some positive $M_{1}$. By utilising the above inequality in (4.6) we infer that

$$
\begin{gathered}
\frac{d}{d t} \Lambda \leq\left(2 M_{1} A_{\varepsilon} u^{\varepsilon}-A_{\varepsilon} \partial_{t} u^{\varepsilon},\left(A_{\varepsilon}^{-1}-A_{0}^{-1}\right) r^{\varepsilon}\right)-2 M_{1}\left(A_{\varepsilon} u^{\varepsilon},\left(A_{\varepsilon}^{-1}-A_{0}^{-1}\right) r^{\varepsilon}\right)+ \\
+M_{1}\left(\frac{1}{2}\left\|r^{\varepsilon}\right\|^{2}+\frac{1}{2}\left(\partial_{t} r^{\varepsilon}, A_{0}^{-1} \partial_{t} r^{\varepsilon}\right)\right) .
\end{gathered}
$$

Now, by the dissipative estimate in $\varepsilon_{\varepsilon}^{2}$ (Theorem 2.5) we have the following uniform bounds in $t$ and $\varepsilon$ :

$$
\left\|A_{\varepsilon} u^{\varepsilon}(t)\right\|+\left\|A_{\varepsilon} \partial_{t} u^{\varepsilon}(t)\right\| \leq M, \quad t \geq 0, \varepsilon>0,
$$


which we use along with the Cauchy-Schwarz inequality to compute

$$
\left|\left(2 M_{1} A_{\varepsilon} u^{\varepsilon}-A_{\varepsilon} \partial_{t} u^{\varepsilon},\left(A_{\varepsilon}^{-1}-A_{0}^{-1}\right) r^{\varepsilon}\right)\right| \leq M\left\|A_{\varepsilon}^{-1}-A_{0}^{-1}\right\|_{\mathcal{L}\left(L^{2}(\Omega)\right)}^{2}+\frac{M_{1}}{2}\left\|r^{\varepsilon}\right\|^{2} .
$$

By collecting the above inequalities together we deduce that

$$
\frac{d}{d t} \Lambda \leq M\left\|A_{\varepsilon}^{-1}-A_{0}^{-1}\right\|_{\mathcal{L}\left(L^{2}(\Omega)\right)}^{2}+2 M_{1} \Lambda .
$$

Consequently, by applying Gronwall's inequality and the initial data $\left.\xi_{r \varepsilon}\right|_{t=0}=0$ we have

$$
\frac{1}{2}\left(\partial_{t} r^{\varepsilon}(t), A_{0}^{-1} \partial_{t} r^{\varepsilon}(t)\right)+\frac{1}{2}\left\|r^{\varepsilon}(t)\right\|^{2}-\left(A_{\varepsilon} u^{\varepsilon}(t),\left(A_{\varepsilon}^{-1}-A_{0}^{-1}\right) r^{\varepsilon}(t)\right) \leq e^{2 M_{1} t} \frac{M}{M_{1}}\left\|A_{\varepsilon}^{-1}-A_{0}^{-1}\right\|_{\mathcal{L}\left(L^{2}(\Omega)\right)}^{2}, \quad t \geq 0 .
$$

Now, we compute

$$
\begin{aligned}
\left|\left(A_{\varepsilon} u^{\varepsilon},\left(A_{\varepsilon}^{-1}-A_{0}^{-1}\right) r^{\varepsilon}\right)\right| & \leq\left\|A_{\varepsilon} u^{\varepsilon}\right\|\left\|A_{\varepsilon}^{-1}-A_{0}^{-1}\right\|_{\mathcal{L}\left(L^{2}(\Omega)\right)}\left\|r^{\varepsilon}\right\| \\
& \leq\left\|A_{\varepsilon} u^{\varepsilon}\right\|^{2}\left\|A_{\varepsilon}^{-1}-A_{0}^{-1}\right\|_{\mathcal{L}\left(L^{2}(\Omega)\right)}^{2}+\frac{1}{4}\left\|r^{\varepsilon}\right\|^{2} .
\end{aligned}
$$

Hence, the above two inequalities along with (4.7) and (4.9) demonstrate (4.3) and the proof is complete.

Along with Theorem 4.2, to prove error estimates on the distance between global attractors we need the following exponential attraction property of $\mathcal{A}^{0}$ :

$$
\left\{\begin{array}{l}
\text { there exists a constant } \sigma>0 \text { such that for every bounded set } B \subset \mathcal{E} \text { the estimate } \\
\operatorname{dist}_{\mathcal{E}}\left(S_{0}(t) B, \mathcal{A}^{0}\right) \leq M\left(\|B\|_{\mathcal{E}}\right) e^{-\sigma t}, \quad t \geq 0, \\
\text { holds for some non-decreasing function } M .
\end{array}\right.
$$

It is known that, for problem (0.2), the property $(\mathrm{H} 2)$ is a generic assumption in the sense that it holds for an open dense subset of forces $g \in L^{2}(\Omega)$ (cf. [2]).

We are now ready to formulate and prove our main result of this section.

Theorem 4.3. Assume (H1) and (H2). Let $\mathcal{A}^{\varepsilon}$ and $\mathcal{A}^{0}$ be the global attractors of the dynamical systems $\left(\mathcal{E}, S_{\varepsilon}(t)\right)$ and $\left(\mathcal{E}, S_{0}(t)\right)$ corresponding to the problems (0.1) and (0.2). Then the following estimate

$$
\operatorname{dist}_{\mathcal{E}^{-1}}\left(\mathcal{A}^{\varepsilon}, \mathcal{A}^{0}\right) \leq M\left\|A_{\varepsilon}^{-1}-A_{0}^{-1}\right\|_{\mathcal{L}\left(L^{2}(\Omega)\right)}^{\mathcal{L}}, \quad \varkappa=\frac{\sigma}{(K+\sigma)},
$$

holds. Here, $K$ is as in Theorem 4.2, $\sigma$ as in (H2), and $M=M(\|g\|)$ is a non-decreasing function independent of $\varepsilon$.

Proof. The assertion follows from the already obtained estimate (4.3) and the exponential attraction property (H2). Indeed, let $\xi_{\varepsilon} \in \mathcal{A}^{\varepsilon} \subset B_{\mathcal{E}_{\varepsilon}^{2}}\left(0, R_{1}\right)$ be arbitrary. Then due to (2.6) there exists a complete bounded trajectory $\xi_{u^{\varepsilon}}(t) \in \mathcal{K}^{\varepsilon}$, such that $\xi_{u^{\varepsilon}}(0)=\xi_{\varepsilon}$. Let us fix an arbitrary $T \geq 0$ and consider $\xi_{-T, \varepsilon}=\xi_{u^{\varepsilon}}(-T) \in \mathcal{A}^{\varepsilon}$. By Theorem 4.2 we deduce

$$
\left\|\xi_{\varepsilon}-S_{0}(T) \xi_{-T, \varepsilon}\right\|_{\mathcal{E}^{-1}} \leq M \kappa e^{K T}, \quad \text { for } \kappa=\left\|A_{\mathcal{\varepsilon}}^{-1}-A_{0}^{-1}\right\|_{\mathcal{L}\left(L^{2}(\Omega)\right)} .
$$

for some $M$ and $K$ which are independent of $\varepsilon$ and $\xi_{\varepsilon} \in \mathcal{A}^{\varepsilon}$. On the other hand, due to exponential attraction (H2) we have

$$
\operatorname{dist}_{\mathcal{E}^{-1}}\left(S_{0}(T) \xi_{-T, \varepsilon}, \mathcal{A}^{0}\right) \leq M e^{-\sigma T} .
$$

Therefore, using the triangle inequality, we derive

$$
\operatorname{dist}_{\mathcal{E}^{-1}}\left(\xi_{\varepsilon}, \mathcal{A}^{0}\right) \leq M\left(\kappa e^{K T}+e^{-\sigma T}\right) .
$$

We recall that $T \geq 0$ is arbitrary and therefore we choose $T$ that minimizes the right hand side of (4.11). For example, taking $T=T(\varepsilon)$ such that $\kappa e^{K T}=e^{-\sigma T}$ yields

$$
\operatorname{dist}_{\mathcal{E}^{-1}}\left(\xi_{\varepsilon}, \mathcal{A}^{0}\right) \leq 2 M\left\|A_{\varepsilon}^{-1}-A_{0}^{-1}\right\|_{\mathcal{L}\left(L^{2}(\Omega)\right)}, \quad \varkappa=\frac{\sigma}{(K+\sigma)},
$$

and since $\xi_{\varepsilon} \in \mathcal{A}^{\varepsilon}$ is arbitrary we obtain the desired inequality (4.10). 
To complement the convergence result in Theorem 3.1, we have the following error estimates.

Corollary 4.1. Assume (H1) and (H2). Let $\alpha>0$ be given by Remark 2.1, $\varkappa$ as in Theorem 4.3 and $0 \leq \beta<\alpha$. Then the inequality

$$
\operatorname{dist}_{\left(C^{\beta}(\bar{\Omega})\right)^{2}}\left(\mathcal{A}^{\varepsilon}, \mathcal{A}^{0}\right) \leq M\left\|A_{\varepsilon}^{-1}-A_{0}^{-1}\right\|_{\mathcal{L}\left(L^{2}(\Omega)\right)}^{\theta \varkappa}, \quad \theta=\frac{\alpha-\beta}{2+\alpha},
$$

for some non-decreasing function $M=M(\|g\|)$ which is independent of $\varepsilon$.

Proof. The corollary follows directly from the uniform boundedness of $\mathcal{A}^{\varepsilon}$ and $\mathcal{A}^{0}$ in $\left(C^{\alpha}(\bar{\Omega})\right)^{2}(\operatorname{Remark} 3.1)$, the estimate on the distance between attractors in $\mathcal{E}^{-1}$ (cf. (4.10)) and the interpolation inequalities

$$
\begin{array}{ll}
\|u\|_{L^{\infty}(\Omega)} \leq C\|u\|_{H^{-1}(\Omega)}^{9}\|u\|_{C^{\alpha}(\bar{\Omega})}^{1-\vartheta}, & \forall u \in H^{-1}(\Omega) \cap C^{\alpha}(\bar{\Omega}), \text { where } \vartheta=\frac{\alpha}{2+\alpha}, \\
\|u\|_{C^{\beta}(\bar{\Omega})} \leq 2\|u\|_{C^{\alpha}(\bar{\Omega})}^{\beta / \alpha}\|u\|_{L^{\infty}(\Omega)}^{(1-\beta / \alpha)}, & \forall u \in C^{\alpha}(\bar{\Omega}) .
\end{array}
$$

\section{Approximation of global attractors with error estimates in the energy space $\mathcal{E}$}

In addition to the obtained estimates in Section 4 on the distance in $\mathcal{E}^{-1}$ we would like to obtain estimates in the energy space $\mathcal{E}$. Note that we can not expect, in general, convergence of the global attractors in the strong topology of $\varepsilon$, cf. Remark 3.2. As in the elliptic case, estimates in $H^{1}(\Omega)$-norm require involving the correction $\varepsilon \sum_{i} N_{i}(\dot{\bar{\varepsilon}}) \partial_{\chi_{i}} u^{0}$ of homogenised trajectories $u^{0}$. To this end, we introduce the 'correction' operator $\mathcal{T}_{\varepsilon}: H^{2}(\Omega) \rightarrow H^{1}(\Omega)$ given by

$$
\mathcal{T}_{\varepsilon} w(x):=w(x)+\varepsilon \sum_{i=1}^{3} N_{i}\left(\frac{x}{\varepsilon}\right) \partial_{x_{i}} w(x), \quad x \in \Omega .
$$

Here, $N_{i}, i \in\{1,2,3\}$, are the solutions to the cell problem (1.1).

Now, it is known that $N_{i}, i=1,2,3$, are multipliers in $H^{1}(\Omega)$ (see [32, Section 13] and [33, Proposition 9.3]); in particular the following non-trivial estimate holds (see [16, Section 3]): there exists $C=C(v, \Omega)$ such that

$$
\int_{\Omega}\left|\nabla_{y} N_{i}\left(\frac{x}{\varepsilon}\right) u(x)\right|^{2} \mathrm{~d} x \leq C \int_{\Omega}\left(|u(x)|^{2}+\varepsilon^{2}|\nabla u(x)|^{2}\right) \mathrm{d} x, \quad \forall u \in H^{1}(\Omega) .
$$

Consequently, the following inequality

$$
\left\|\nabla \mathcal{T}_{\varepsilon} w\right\| \leq C\left(\|\nabla w\|+\varepsilon\|w\|_{H^{2}(\Omega)}\right), \quad \forall w \in H^{2}(\Omega),
$$

holds for some $C>0$ independent of $\varepsilon$ and $w$. Indeed, this follows from the above multiplier estimate and the fact $N_{i} \in L^{\infty}(Q)$ (by elliptic regularity).

Now, we are ready to present the well-known corrector estimate result in elliptic homogenisation theory which improves the $L^{2}$-estimate given in Theorem 4.1 to $H^{1}$-norm.

Theorem 5.1 (Theorem 3.1, [16]). Let $\Omega \subset \mathbb{R}^{3}$ be a bounded smooth domain, periodic matrix a $(\cdot)$ satisfying uniform elliptic and boundedness assumptions, $A_{\varepsilon}$ and $A_{0}$ given by (4.1) and $g \in L^{2}(\Omega)$. Let also $u^{\varepsilon}, u^{0} \in H_{0}^{1}(\Omega)$ solve the problems

$$
\left\{\begin{array} { l } 
{ A _ { \varepsilon } u ^ { \varepsilon } = g , \quad \text { in } \Omega , } \\
{ u ^ { \varepsilon } | _ { \partial \Omega } = 0 , }
\end{array} \quad \& \quad \left\{\begin{array}{l}
A_{0} u^{0}=g, \quad \text { in } \Omega, \\
\left.u^{0}\right|_{\partial \Omega}=0 .
\end{array}\right.\right.
$$

Then, the following estimate

$$
\left\|u^{\varepsilon}-\mathcal{T}_{\varepsilon} u^{0}\right\|_{H_{0}^{1}(\Omega)} \leq C \sqrt{\varepsilon}\|g\|
$$

holds for some constant $C=C(v, \Omega)$. 
Remark 5.1. Note that inequality (5.3) is equivalent to the following operator estimate:

$$
\left\|A_{\varepsilon}^{-1} g-\mathcal{T}_{\varepsilon} A_{0}^{-1} g\right\|_{H_{0}^{1}(\Omega)} \leq C \sqrt{\varepsilon}\|g\|, \quad g \in L^{2}(\Omega) .
$$

As in Theorem 4.3, we would like to compare the distance between $S_{\varepsilon}(t) \xi$, for $\xi \in \mathcal{E}_{\varepsilon}^{2}$, to some trajectory for $S_{0}$ but this time in the energy space $\mathcal{E}$. However, here the trajectory $S_{0}(t) \xi$ is not a suitable candidate as it does not have the sufficient regularity needed to apply the above corrector estimates. To overcome this difficulty we carefully choose our initial data for the homogenised problem (0.2).

More precisely, let us recall the spaces $\varepsilon_{\varepsilon}^{2}, \varepsilon_{0}^{2}$ given in (3.1), (3.2), and introduce the bounded linear operator $\Pi_{\varepsilon}: \varepsilon_{\varepsilon}^{2} \rightarrow \varepsilon_{0}^{2}$ given by

$$
\Pi_{\varepsilon}\left(\xi^{1}, \xi^{2}\right):=\left(\xi_{0}^{1}, \xi_{0}^{2}\right), \quad \text { where } \quad\left\{\begin{array}{l}
\text { the term } \xi_{0}^{i} \in H^{2}(\Omega) \cap H_{0}^{1}(\Omega), i=1,2, \text { satisfies } \\
\operatorname{div}\left(a^{h} \nabla \xi_{0}^{i}\right)=\operatorname{div}\left(a(\dot{\bar{\varepsilon}}) \nabla \xi^{i}\right) .
\end{array}\right.
$$

The operator $\Pi_{\varepsilon}$ has the following nice properties.

Lemma 5.1. The operator $\Pi_{\varepsilon}: \varepsilon_{\varepsilon}^{2} \rightarrow \varepsilon_{0}^{2}$ is a bijection that satisfies:

$$
\begin{array}{ll}
\left\|\Pi_{\varepsilon} \xi\right\|_{\mathcal{E}_{0}^{2}}=\|\xi\|_{\mathcal{E}_{\varepsilon}^{2}}, & \xi \in \mathcal{E}_{\varepsilon}^{2} \\
\left\|\Pi_{\varepsilon} \xi-\xi\right\|_{\left(L^{2}(\Omega)\right)^{2}} \leq\left\|A_{\varepsilon}^{-1}-A_{0}^{-1}\right\|_{\mathcal{L}\left(L^{2}(\Omega)\right)}\|\xi\|_{\mathcal{E}_{\varepsilon}^{2}}, & \xi \in \mathcal{E}_{\varepsilon}^{2}
\end{array}
$$

Proof. The bijective property and equality (5.5) directly follow from the definitions of $\varepsilon_{\varepsilon}^{2}$, $\varepsilon_{0}^{2}$ and the identity $\Pi_{\varepsilon}\left(\xi^{1}, \xi^{2}\right)=\left(A_{0}^{-1} A_{\varepsilon} \xi^{1}, A_{0}^{-1} A_{\varepsilon} \xi^{2}\right)$. Inequality (5.6) follows from the identity

$$
A_{0}^{-1} A_{\varepsilon} \xi^{i}-\xi^{i}=\left(A_{0}^{-1}-A_{\varepsilon}^{-1}\right) A_{\varepsilon} \xi^{i} .
$$

We now compare $S_{\varepsilon}(t) \xi$ with $S_{0}(t) \Pi_{\varepsilon} \xi$ in $\varepsilon$ for $\xi \in \mathcal{E}_{\varepsilon}^{2}$. The following result is the direct analogue of Theorem 4.2 when one replaces the initial data $\xi$ by $\Pi_{\varepsilon} \xi$ in problem (0.2).

Theorem 5.2. Let $\varepsilon_{\varepsilon}^{2}$ be the set (3.1). Then, for every $\xi \in B_{\mathcal{E}_{\varepsilon}^{2}}(0, R)$, the following inequalities

$$
\begin{aligned}
& \left\|S_{\varepsilon}(t) \xi-S_{0}(t) \Pi_{\varepsilon} \xi\right\|_{\mathcal{E}^{-1}} \leq M e^{K t}\left\|A_{\varepsilon}^{-1}-A_{0}^{-1}\right\|_{\mathcal{L}\left(L^{2}(\Omega)\right)}, \quad t \geq 0, \\
& \left\|\partial_{t} S_{\varepsilon}(t) \xi-\partial_{t} S_{0}(t) \Pi_{\varepsilon} \xi\right\|_{\mathcal{E}^{-1}} \leq M e^{K t}\left\|A_{\varepsilon}^{-1}-A_{0}^{-1}\right\|_{\mathcal{L}\left(L^{2}(\Omega)\right)}^{1 / 2}, \quad t \geq 0,
\end{aligned}
$$

hold for some non-decreasing functions $M=M(R,\|g\|)$ and $K=K(R,\|g\|)$ which are independent of $\varepsilon>0$.

Proof. First note that inequality (5.7) is a consequence of the Lipschitz continuity of $S_{0}$ in $\mathcal{E}^{-1}$ (Corollary 1.2), Lemma 5.1 and (4.3). Indeed,

$$
\begin{aligned}
\left\|S_{\varepsilon}(t) \xi-S_{0}(t) \Pi_{\varepsilon} \xi\right\|_{\mathcal{E}^{-1}} & \leq\left\|S_{\varepsilon}(t) \xi-S_{0}(t) \xi\right\|_{\mathcal{E}^{-1}}+\left\|S_{0}(t) \xi-S_{0}(t) \Pi_{\varepsilon} \xi\right\|_{\mathcal{E}^{-1}} \\
& \leq\left\|S_{\varepsilon}(t) \xi-S_{0}(t) \xi\right\|_{\mathcal{E}^{-1}}+M e^{K t}\left\|\xi-\Pi_{\varepsilon} \xi\right\|_{\mathcal{E}^{-1}} \leq M e^{K t}\left\|A_{\varepsilon}^{-1}-A_{0}^{-1}\right\|_{\mathcal{L}\left(L^{2}(\Omega)\right)} .
\end{aligned}
$$

It remains to prove (5.8).

Set $\xi_{u^{\varepsilon}}(t):=S_{\varepsilon}(t) \xi, \xi_{u^{0}}(t):=S_{0}(t) \Pi_{\varepsilon} \xi$. We begin by noting the following uniform bounds in $t$ and $\varepsilon$ :

$$
\left\|\partial_{t}^{2} u^{\varepsilon}\right\|+\left\|\nabla u^{\varepsilon}\right\|+\left\|A_{\varepsilon} \partial_{t} u^{\varepsilon}\right\|+\left\|\partial_{t}^{2} u^{0}\right\|+\left\|\nabla u^{0}\right\| \leq M
$$

Indeed, these bounds are a consequence of identity $\Pi_{\varepsilon} B_{\varepsilon_{\varepsilon}^{2}}(0, R)=B_{\mathcal{E}_{0}^{2}}(0, R)$ and the dissipative estimates for $u^{\varepsilon}$ and $u^{0}$ in $\varepsilon_{\varepsilon}^{2}$ and $\varepsilon_{0}^{2}$ respectively (Theorem 2.5 for $a=a\left(\frac{\dot{\bar{\varepsilon}}}{\varepsilon}\right)$ and $a=a^{h}$ respectively).

Now, the difference $r^{\varepsilon}:=u^{\varepsilon}-u^{0}$ solves

$$
\left\{\begin{array}{l}
\partial_{t}^{2} r^{\varepsilon}=-y \partial_{t} r^{\varepsilon}+A_{0} u^{0}-A_{\varepsilon} u^{\varepsilon}+f\left(u^{0}\right)-f\left(u^{\varepsilon}\right), \quad x \in \Omega, t \geq 0, \\
\left.\xi_{r^{\varepsilon}}\right|_{t=0}=\xi-\Pi_{\varepsilon} \xi,\left.\quad r^{\varepsilon}\right|_{\partial \Omega}=0 .
\end{array}\right.
$$


Note that by the definition of $\Pi_{\varepsilon}$, (5.4), we have

$$
\left.\xi_{\partial_{t} r}\right|_{t=0}=\left(\xi^{2}-\xi_{0}^{2}, y\left(\xi_{0}^{2}-\xi^{2}\right)+f\left(\xi_{0}^{1}\right)-f\left(\xi^{1}\right)\right) .
$$

Upon handling the non-linearity as in (4.8), and utilising Lemma 5.1 we conclude that

$$
\left\|\xi_{\partial_{t} r^{\varepsilon}}(0)\right\|_{\mathcal{E}^{-1}} \leq C\left\|A_{\varepsilon}^{-1}-A_{0}^{-1}\right\|_{\mathcal{L}\left(L^{2}(\Omega)\right)} .
$$

Now, by differentiating the first equation in (5.10) in time (and then adding $A_{0} \partial_{t} r^{\varepsilon}$ to both sides) we find that $q^{\varepsilon}:=\partial_{t} r^{\varepsilon}$ solves

$$
\left\{\begin{array}{l}
\partial_{t}^{2} q^{\varepsilon}+y \partial_{t} q^{\varepsilon}+A_{0} q^{\varepsilon}=A_{0} \partial_{t} u^{\varepsilon}-A_{\varepsilon} \partial_{t} u^{\varepsilon}+f^{\prime}\left(u^{0}\right) \partial_{t} u^{0}-f^{\prime}\left(u^{\varepsilon}\right) \partial_{t} u^{\varepsilon}, \quad x \in \Omega, t \geq 0, \\
\left.\xi_{q^{\varepsilon}}\right|_{t=0}=\xi_{\partial_{t} r^{\varepsilon}}(0),\left.\quad q^{\varepsilon}\right|_{\partial \Omega}=0 .
\end{array}\right.
$$

Testing the first equation in the above problem with $A_{0}^{-1} \partial_{t} q^{\varepsilon}$ gives

$$
\begin{aligned}
\frac{d}{d t}\left(\frac{1}{2}\left(\partial_{t} q^{\varepsilon}, A_{0}^{-1} \partial_{t} q^{\varepsilon}\right)+\frac{1}{2}\left\|q^{\varepsilon}\right\|^{2}\right)+y & \left(\partial_{t} q^{\varepsilon}, A_{0}^{-1} \partial_{t} q^{\varepsilon}\right)= \\
& \left(A_{0} \partial_{t} u^{\varepsilon}-A_{\varepsilon} \partial_{t} u^{\varepsilon}, A_{0}^{-1} \partial_{t} q^{\varepsilon}\right)+\left(f^{\prime}\left(u^{0}\right) \partial_{t} u^{0}-f^{\prime}\left(u^{\varepsilon}\right) \partial_{t} u^{\varepsilon}, A_{0}^{-1} \partial_{t} q^{\varepsilon}\right) .
\end{aligned}
$$

We aim to prove the inequality

$$
\frac{d}{d t} \Lambda \leq M e^{K t}\left\|A_{\varepsilon}^{-1}-A_{0}^{-1}\right\|_{\mathcal{L}\left(L^{2}(\Omega)\right)}+M \Lambda, \quad \Lambda:=\frac{1}{2}\left(\partial_{t} q^{\varepsilon}, A_{0}^{-1} \partial_{t} q^{\varepsilon}\right)+\frac{1}{2}\left\|q^{\varepsilon}\right\|^{2}
$$

for some $M$ and $K$ independent of $\varepsilon$ and $\xi_{0}$, which subsequently implies the desired result via an application of Gronwall's inequality and (5.11). As usual, we shall utilise the $H^{-1}$-norm equivalence given by (4.7).

So it remains to prove (5.12). By arguing as in Theorem 4.2, we utilise the identity $\partial_{t} q^{\varepsilon}=\partial_{t}^{2} u^{\varepsilon}-\partial_{t}^{2} u^{0}$ and uniform bounds (5.9) to compute

$$
\begin{aligned}
\left|\left(A_{0} \partial_{t} u^{\varepsilon}-A_{\varepsilon} \partial_{t} u^{\varepsilon}, A_{0}^{-1} \partial_{t} q^{\varepsilon}\right)\right| & =\left|\left(A_{\varepsilon} \partial_{t} u^{\varepsilon},\left(A_{\varepsilon}^{-1}-A_{0}^{-1}\right) \partial_{t} q^{\varepsilon}\right)\right| \\
& \leq\left\|A_{\varepsilon} \partial_{t} u^{\varepsilon}\right\|\left\|A_{\varepsilon}^{-1}-A_{0}^{-1}\right\|_{\mathcal{L}\left(L^{2}(\Omega)\right)}\left\|\partial_{t} q^{\varepsilon}\right\| \\
& \leq M\left\|A_{\varepsilon}^{-1}-A_{0}^{-1}\right\|_{\mathcal{L}\left(L^{2}(\Omega)\right)} .
\end{aligned}
$$

Let us now handle the non-linear term. We compute

$$
\left(f^{\prime}\left(u^{0}\right) \partial_{t} u^{0}-f^{\prime}\left(u^{\varepsilon}\right) \partial_{t} u^{\varepsilon}, A_{0}^{-1} \partial_{t} q^{\varepsilon}\right)=-\left(f^{\prime}\left(u^{0}\right) q^{\varepsilon}, A_{0}^{-1} \partial_{t} q^{\varepsilon}\right)+\left(\left(f^{\prime}\left(u^{0}\right)-f^{\prime}\left(u^{\varepsilon}\right)\right) \partial_{t} u^{\varepsilon}, A_{0}^{-1} \partial_{t} q^{\varepsilon}\right)=: I_{1}+I_{2} .
$$

The arguments to bound $I_{1}$ and $I_{2}$ will use the uniform bounds on $u^{\varepsilon}$ and $u^{0}$ given by (5.9).

By the growth condition on $f$ and the $H^{-1}$-norm equivalence (4.7), we compute

$$
\begin{aligned}
\left|I_{1}\right|=\left|\left(f^{\prime}\left(u^{0}\right) q^{\varepsilon}, A_{0}^{-1} \partial_{t} q^{\varepsilon}\right)\right| & \leq M\left(\left(1+\left|u^{0}\right|^{2}\right)\left|q^{\varepsilon}\right|,\left|A_{0}^{-1} \partial_{t} q^{\varepsilon}\right|\right) \leq M\left\|1+\left|u^{0}\right|^{2}\right\|_{L^{3}(\Omega)}\left\|q^{\varepsilon}\right\|\left\|A_{0}^{-1} \partial_{t} q^{\varepsilon}\right\|_{L^{6}(\Omega)} \\
& \leq M\left\|q^{\varepsilon}\right\|\left\|\partial_{t} q^{\varepsilon}\right\|_{H^{-1}(\Omega)} \leq M\left(\frac{1}{2}\left\|q^{\varepsilon}\right\|^{2}+\frac{1}{2}\left(\partial_{t} q^{\varepsilon}, A_{0}^{-1} \partial_{t} q^{\varepsilon}\right)\right) .
\end{aligned}
$$

Additionally, by Hölder's inequality (for exponents $\left.\left(p_{1}, p_{2}, p_{3}, p_{4}\right)=(6,2,6,6)\right)$ we compute

$$
\begin{aligned}
\left|I_{2}\right|=\left|\left(\left(f^{\prime}\left(u^{0}\right)-f^{\prime}\left(u^{\varepsilon}\right)\right) \partial_{t} u^{\varepsilon}, A_{0}^{-1} \partial_{t} q^{\varepsilon}\right)\right| & \leq M\left(\left(1+\left|u^{0}\right|+\left|u^{\varepsilon}\right|\right)\left|r^{\varepsilon} \| \partial_{t} u^{\varepsilon}\right|,\left|A_{0}^{-1} \partial_{t} q^{\varepsilon}\right|\right) \\
& \leq M\left\|1+\left|u^{0}\right|+\left|u^{\varepsilon}\right|\right\|_{L^{6}(\Omega)}\left\|r^{\varepsilon}\right\|\left\|\partial_{t} u^{\varepsilon}\right\|_{L^{6}(\Omega)}\left\|A_{0}^{-1} \partial_{t} q^{\varepsilon}\right\|_{L^{6}(\Omega)} \\
& \leq M\left(\frac{1}{2}\left\|r^{\varepsilon}\right\|^{2}+\frac{1}{2}\left(\partial_{t} q^{\varepsilon}, A_{0}^{-1} \partial_{t} q^{\varepsilon}\right)\right) .
\end{aligned}
$$

The above assertion and (5.7) imply

$$
\left|I_{2}\right| \leq C\left(e^{2 K t}\left\|A_{\varepsilon}^{-1}-A_{0}^{-1}\right\|_{\mathcal{L}\left(L^{2}(\Omega)\right)}^{2}+\frac{1}{2}\left(\partial_{t} q^{\varepsilon}, A_{0}^{-1} \partial_{t} q^{\varepsilon}\right)\right) .
$$

Combining the above calculations leads to the inequality (5.12). The proof is complete.

The following estimate is an immediate consequence of Theorem 5.2 and standard elliptic theory. 
Corollary 5.1. Let $\mathcal{E}_{\varepsilon}^{2}$ be the set (3.1), $\xi \in B_{\mathcal{E}_{\varepsilon}^{2}}(0, R)$ and set $\xi_{u^{\varepsilon}}(t):=S_{\varepsilon}(t) \xi, \xi_{u^{0}}(t):=S_{0}(t) \Pi_{\varepsilon} \xi$. Let $\mathcal{T}_{\varepsilon}$ be given by (5.1). Then, the following inequality

$$
\left\|u^{\varepsilon}(t)-\mathcal{T}_{\varepsilon} u^{0}(t)\right\|_{H^{1}(\Omega)} \leq M e^{K t} \sqrt{\varepsilon}, \quad t \geq 0,
$$

holds for some non-decreasing $M=M(R,\|g\|)$ and $K=K(R,\|g\|)$ which are independent of $\varepsilon>0$.

Proof. Note that $u^{\varepsilon} \in H_{0}^{1}(\Omega)$ satisfies the equation

$$
A_{\varepsilon} u^{\varepsilon}=-\partial_{t}^{2} u^{\varepsilon}-y \partial_{t} u^{\varepsilon}-f\left(u^{\varepsilon}\right)+g=: F_{\varepsilon}(t), \quad t \geq 0,
$$

and $u^{0} \in H_{0}^{1}(\Omega)$ satisfies

$$
A_{0} u^{0}=-\partial_{t}^{2} u^{0}-y \partial_{t} u^{0}-f\left(u^{0}\right)+g=: F_{0}(t), \quad t \geq 0 .
$$

Since $\xi \in B_{\varepsilon_{\varepsilon}^{2}}(0, R)$ then by (5.5) we have $\Pi_{\varepsilon} \xi \in B_{\varepsilon_{0}^{2}}(0, R)$ and the dissipative estimate in $\varepsilon_{0}^{2}$ (Theorem 2.5 for $a=a^{h}$ ) gives $F_{0} \in L^{\infty}\left(\mathbb{R}_{+} ; L^{2}(\Omega)\right)$. Let us introduce the intermediate function $\widetilde{u}_{\varepsilon}=\widetilde{u}^{\varepsilon}(t) \in H_{0}^{1}(\Omega)$ the solution to

$$
A_{\varepsilon} \tilde{u}^{\varepsilon}=F_{0}(t), \quad t \geq 0 .
$$

Then, by Theorem 5.1 we have

$$
\left\|\tilde{u}^{\varepsilon}(t)-\mathcal{T}_{\mathcal{\varepsilon}} u^{0}(t)\right\|_{H_{0}^{1}(\Omega)} \leq C \sqrt{\varepsilon}\left\|F_{0}(t)\right\|, \quad t \geq 0,
$$

and, since $A_{\varepsilon}^{-1}$ is uniformly bounded in $\mathcal{L}\left(H^{-1}(\Omega), H_{0}^{1}(\Omega)\right)$, we have

$$
\left\|u^{\varepsilon}(t)-\tilde{u}^{\varepsilon}(t)\right\|_{H_{0}^{1}(\Omega)} \leq C\left\|F_{\varepsilon}(t)-F_{0}(t)\right\|_{H^{-1}(\Omega)}, \quad t \geq 0 .
$$

Therefore, by the triangle inequality, we have

$$
\left\|u^{\varepsilon}(t)-\mathcal{T}_{\varepsilon} u^{0}(t)\right\|_{H_{0}^{1}(\Omega)} \leq C\left(\sqrt{\varepsilon}\left\|F_{0}\right\|_{L^{\infty}\left(\mathbb{R}_{+} ; L^{2}(\Omega)\right)}+\left\|F_{\varepsilon}(t)-F_{0}(t)\right\|_{H^{-1}(\Omega)}\right), \quad t \geq 0 .
$$

Now, upon estimating the non-linear term as in the proof of Theorem 5.2, along with utilising Remark 4.1 and Theorem 5.2, we readily deduce that

$$
\left\|F_{\varepsilon}(t)-F_{0}(t)\right\|_{H^{-1}(\Omega)} \leq M e^{K t} \sqrt{\varepsilon}, \quad t \geq 0 .
$$

The above inequality along with (5.15) imply the desired result and the proof is complete.

Let us now provide estimates on the distance in the energy space. As in Corollary 5.1 this requires adding an appropriate correction to the attractor $\mathcal{A}^{0}$. To this end, we introduce the corrector $\mathrm{T}_{\varepsilon}: \varepsilon_{0}^{2} \rightarrow\left(L^{2}(\Omega)\right)^{2}$ which maps the pair $\xi=\left(\xi^{1}, \xi^{2}\right)$ to the pair

$$
\mathrm{T}_{\varepsilon} \xi=\left(\mathcal{T}_{\varepsilon} \xi^{1}, \xi^{2}\right) .
$$

By (5.2), we readily deduce the following inequality: there exists a constant $C>0$, independent of $\varepsilon$, such that the inequality

$$
\operatorname{dist}_{\mathcal{E}}^{S}\left(\mathrm{~T}_{\varepsilon} A, \mathrm{~T}_{\varepsilon} B\right) \leq C\left(\operatorname{dist}_{\mathcal{E}}^{S}(A, B)+\varepsilon \operatorname{dist}_{\mathcal{E}_{0}^{2}}^{S}(A, B)\right), \quad A, B \subset \mathcal{E}_{0}^{2},
$$

holds.

By inequality (5.8) and Corollary 5.1 we have shown the following result.

Corollary 5.2. Let $\varepsilon_{\varepsilon}^{2}$ be the set (3.1), $\xi \in B_{\mathcal{E}_{\varepsilon}^{2}}(0, R)$ and set $\xi_{u^{\varepsilon}}(t):=S_{\varepsilon}(t) \xi, \xi_{u^{0}}(t):=S_{0}(t) \Pi_{\varepsilon} \xi$. Then, the inequality

$$
\left\|S_{\varepsilon}(t) \xi-T_{\varepsilon} S_{0}(t) \Pi_{\varepsilon} \xi\right\|_{\varepsilon} \leq M e^{K t} \sqrt{\varepsilon},
$$

holds for some non-decreasing $M=M(R,\|g\|)$ and $K=K(R,\|g\|)$ independent of $\varepsilon$. 
The following estimate on the global attractors in $\mathcal{E}$ holds.

Theorem 5.3. Assume (H1) and (H2). Let $\mathcal{A}^{\varepsilon}$ and $\mathcal{A}^{0}$ the global attractors of problems (0.1) and (0.2) respectively, and let $T_{\varepsilon}$ be given by (5.16). Then, the following estimate

$$
\operatorname{dist}_{\varepsilon}\left(\mathcal{A}^{\varepsilon}, T_{\varepsilon} \mathcal{A}^{0}\right) \leq M \sqrt{\varepsilon}^{\varkappa},
$$

holds for some $M=M(\|g\|)$ which is independent of $\varepsilon$. Here $\varkappa$ is as in Theorem 4.3.

Proof. The method of proof follows along the same lines as the argument for Theorem 4.3 and so we shall only sketch it here.

For $\xi_{\varepsilon} \in \mathcal{A}^{\varepsilon}$ and $T>0$, consider $\xi_{-T, \varepsilon} \in \mathcal{A}^{\varepsilon}$ that satisfies $S_{\varepsilon}(T) \xi_{-T, \varepsilon}=\xi_{0}$. Then, by Corollary 5.2 we have

$$
\left\|\xi_{\varepsilon}-\mathrm{T}_{\varepsilon} S_{0}(T) \Pi_{\varepsilon} \xi_{-T, \varepsilon}\right\|_{\varepsilon} \leq M e^{K T} \sqrt{\varepsilon} .
$$

Furthermore, by (5.17) we have

$$
\operatorname{dist}_{\mathcal{E}}\left(\mathrm{T}_{\varepsilon} S_{0}(T) \Pi_{\varepsilon} \xi_{-T, \varepsilon}, \mathrm{T}_{\varepsilon} \mathcal{A}^{0}\right) \leq C\left(\operatorname{dist}_{\mathcal{E}}\left(S_{0}(T) \Pi_{\varepsilon} \xi_{-T, \varepsilon}, \mathcal{A}^{0}\right)+\varepsilon \operatorname{dist}_{\mathcal{E}_{0}^{2}}\left(S_{0}(T) \Pi_{\varepsilon} \xi_{-T, \varepsilon}, \mathcal{A}^{0}\right)\right) .
$$

Now, to control the second term on the above right we use the fact that $\Pi_{\varepsilon} \mathcal{A}^{\varepsilon}$ and $\mathcal{A}^{0}$ are bounded subsets of $\varepsilon_{0}^{2}$ (see Remark 3.1 and inequality (5.5)) and that we have a dissipative estimate for $S_{0}(t)$ on $\mathcal{E}_{0}^{2}$ (see Theorem 2.5). Consequently, we compute

$$
\begin{aligned}
\operatorname{dist}_{\mathcal{E}}\left(\xi_{\varepsilon}, \mathrm{T}_{\varepsilon} \mathcal{A}^{0}\right) & \leq \operatorname{dist}_{\mathcal{E}}\left(\xi_{\varepsilon}, \mathrm{T}_{\varepsilon} S_{0}(T) \Pi_{\varepsilon} \xi_{-T, \varepsilon}\right)+\operatorname{dist}_{\mathcal{E}}\left(\mathrm{T}_{\varepsilon} S_{0}(T) \Pi_{\varepsilon} \xi_{-T, \varepsilon}, \mathrm{T}_{\varepsilon} \mathcal{A}^{0}\right) \\
& \leq M_{1} e^{K T} \sqrt{\varepsilon}+M_{2} \operatorname{dist}_{\mathcal{E}}\left(S_{0}(T) \Pi_{\varepsilon} \xi_{-T, \varepsilon}, \mathcal{A}^{0}\right)
\end{aligned}
$$

and the remainder of the proof utilises the exponential attraction property of $\mathcal{A}^{0}$, as in Theorem 4.3.

\section{Remark 5.2.}

1. The appearance of $\sqrt{\varepsilon}$ in (5.3) is a well-known consequence of the fact that the correction $\mathcal{T}_{\varepsilon} u^{0}$ does not approximate well the function $u^{\varepsilon}$ in a $\varepsilon$-neighbourhood of the boundary. In particular, the reduced power of $\varepsilon$ appears in the estimate due to the fact that $\mathcal{T}_{\varepsilon} u^{0}$ does not satisfy the Dirichlet boundary conditions and a 'boundary correction' is needed. In general, the explicit $\varepsilon$-dependence (i.e. leading-order asymptotics) of this boundary correction is not known.

2. In certain situations, such as when $\Omega$ is the whole space or a torus (see Remark 7.2), there is no need for the boundary correction and, consequently, the error estimate (5.3) is order $\varepsilon$. In such situations we expect order $\varepsilon^{\varkappa}$ in our estimate on the distance between global attractors in $\mathcal{E}$ (Theorem 5.3). As it stands, our argument does not provide such an estimate and this is because the power in the right-hand side of (5.8) is not optimal. This is consciously done to avoid unnecessary complications and we provide an argument in Appendix D that gives the expected power.

3. Let us return to Remark 3.2. In this case it is interesting to note that estimate (5.3) is order $\varepsilon$. This is simply because the cell solutions $N_{i}$ are trivial $\left(N_{i} \equiv 0\right)$ and there is no need for boundary corrections; indeed, this can be readily seen by noting that the right-hand-side in problem (1.1) is zero in this situation. Consequently $\mathcal{T}_{\varepsilon}=I$ and (under the refinement in Appendix D) we have the following improvement of Theorem 5.3:

$$
\operatorname{dist}_{\mathcal{E}}\left(\mathcal{A}^{\varepsilon}, \mathcal{A}^{0}\right) \leq M \varepsilon^{\varkappa} .
$$

\section{Exponential attractors: existence, homogenisation and convergence rates}

Let us recall the definition of an exponential attractor for a dynamical system. 
Definition 6.1. Let $S(t): \mathcal{E} \rightarrow \mathcal{E}, t \geq 0$, be a semi-group acting on a Banach space $\mathcal{E}$. Then a set $\mathcal{M}$ is called an exponential attractor for the dynamical system $(\mathcal{E}, S(t))$ if it possesses the following properties:

1. The set $\mathcal{M}$ is compact in $\mathcal{E}$ with finite fractal (box-counting) dimension $\operatorname{dim}_{f}(\mathcal{M}, \mathcal{E})$;

2. The set $\mathcal{M}$ is positively invariant:

$$
S(t) \mathcal{M} \subset \mathcal{M}, \quad \forall t \geq 0 ;
$$

3. The set $\mathcal{M}$ exponentially attracts every bounded set $B$ of $\mathcal{E}$, that is

$$
\operatorname{dist}_{\mathcal{E}}(S(t) B, \mathcal{M}) \leq M\left(\|B\|_{\mathcal{E}}\right) e^{-\sigma t}, \quad t \geq 0,
$$

for some non-decreasing $M$ and constant $\sigma>0$.

\subsection{Existence of exponential attractors and continuity in $\mathcal{E}^{-1}$}

Let us present our main result for this subsection.

Theorem 6.1. Assume (H1). Then, the dynamical systems $\left(\varepsilon, S_{\varepsilon}(t)\right), \varepsilon>0$ and $\left(\varepsilon, S_{0}(t)\right)$ generated by problems (0.1) and (0.2) respectively possess exponential attractors $\mathcal{M}^{\varepsilon}, \mathcal{M}^{0} \subset\left(H_{0}^{1}(\Omega)\right)^{2}$ such that the following properties hold:

1. $\left\|\operatorname{div}\left(a(\dot{\bar{\varepsilon}}) \nabla \xi^{1}\right)+g\right\|_{H_{0}^{1}(\Omega)}+\left\|\operatorname{div}\left(a(\dot{\bar{\varepsilon}}) \nabla \xi^{2}\right)\right\|+\|\xi\|_{\left(C^{\alpha}(\bar{\Omega})\right)^{2}} \leq M(\|g\|)$, for all $\xi=\left(\xi^{1}, \xi^{2}\right) \in \mathcal{M}^{\varepsilon} ;$

2. $\left\|\operatorname{div}\left(a^{h} \nabla \xi^{1}\right)+g\right\|_{H_{0}^{1}(\Omega)}+\left\|\operatorname{div}\left(a^{h} \nabla \xi^{2}\right)\right\|+\|\xi\|_{\left(H^{2}(\Omega)\right)^{2}} \leq M(\|g\|)$, for all $\xi=\left(\xi^{1}, \xi^{2}\right) \in \mathcal{M} \mathbb{N}^{0}$;

3. For every bounded set $B \subset \mathcal{E}$ one has

4.

$$
\operatorname{dist}_{\mathcal{E}}\left(S_{\varepsilon}(t) B, \mathcal{M}^{\mathcal{\varepsilon}}\right)+\operatorname{dist}_{\mathcal{E}}\left(S_{0}(t) B, \mathcal{M}^{0}\right) \leq M(\|B\| \varepsilon) e^{-\sigma t}, \quad t \geq 0 ;
$$

5.

$$
\begin{gathered}
\operatorname{dim}_{f}\left(\mathcal{M}^{\varepsilon}, \mathcal{E}\right)+\operatorname{dim}_{f}\left(\mathcal{M}^{0}, \mathcal{E}\right) \leq D ; \\
\operatorname{dist}_{\mathcal{E}^{-1}}^{s}\left(\mathcal{M}^{\varepsilon}, \mathcal{M}{ }^{0}\right) \leq M\left\|A_{\varepsilon}^{-1}-A_{0}^{-1}\right\|_{\mathcal{L}\left(L^{2}(\Omega)\right)}^{\varkappa} .
\end{gathered}
$$

Here $\alpha$ is the same as in Remark 2.1 and the constants $M>0, \sigma>0,0<\varkappa<1$ and $D \geq 0$ are independent of $\varepsilon$.

Corollary 6.1. Assume (H1). Let $\alpha>0$ be given by Remark 2.1, $\varkappa$ as in Theorem 6.1 and $0 \leq \beta<\alpha$. Then the inequality

$$
\operatorname{dist}_{\left(C^{\beta}(\bar{\Omega})\right)^{2}}^{S}\left(\mathcal{M H}^{\varepsilon}, \mathcal{M}^{0}\right) \leq M\left\|A_{\varepsilon}^{-1}-A_{0}^{-1}\right\|_{\mathcal{L}\left(L^{2}(\Omega)\right)}^{\theta \varkappa}, \quad \theta=\frac{\alpha-\beta}{2+\alpha},
$$

for some non-decreasing function $M=M(\|g\|)$ which is independent of $\varepsilon$.

The remainder of the section is dedicated to the proof of Theorem 6.1. First, we recall a variation of an abstract result which establishes the existence of an exponential attractor $\mathcal{M}^{\varepsilon}$, for a parameter-dependent family of semi-groups $S_{\varepsilon}$, whose characteristics are independent of $\varepsilon$ (see Appendix B, [29, Theorem 2.10] and [30, Section 3, Theorem 3.1]).

Theorem 6.2. Let $\mathcal{E}$ be a Banach space and $\varepsilon_{\varepsilon}^{1}, \varepsilon \geq 0$, be a family of Banach spaces compactly embedded into $\varepsilon$ uniformly in the following sense:

(i) There exists $c_{0}$ independent of $\varepsilon \geq 0$ such that $\|\xi\|_{\mathcal{E}} \leq c_{0}\|\xi\|_{\mathcal{E}_{\varepsilon}^{1}}$ for all $\xi \in \mathcal{E}_{\varepsilon}^{1}$;

(ii) For all $\mu>0, r>0$ there exists a finite cover of $B_{\mathcal{E}_{\varepsilon}^{1}}(0, r)$ consisting of balls radius of $\mu$ in $\mathcal{E}$ with centers $\mathcal{U}_{\varepsilon}(\mu, r) \subset B_{\mathcal{E}_{\varepsilon}^{1}}\left(0, \delta_{r}\right)$, for some $\delta_{r} \geq r$, satisfying

$$
\operatorname{card} \mathcal{U}_{\varepsilon}(\mu, r) \leq N(\mu, r),
$$

for some finite $N(\mu, r)$ independent of $\varepsilon$. 
Let us consider, for each $\varepsilon \geq 0$, a map defined on $\mathcal{\varepsilon}$ such that

$$
S_{\varepsilon}: \mathcal{O}\left(B_{\varepsilon}\right) \rightarrow B_{\varepsilon}, \quad \mathcal{O}\left(B_{\varepsilon}\right):=\left(B_{\varepsilon}+\bigcup_{r \in[0,1]} r \mathcal{U}_{\varepsilon}\left(\frac{1}{4 K}, 1\right)\right) \bigcup \mathcal{U}_{\varepsilon}\left(\frac{1}{K}, R\right),
$$

where the set $B_{\varepsilon} \subset B_{\mathcal{E}_{\varepsilon}^{1}}(0, R)$ is closed in $\mathcal{E}$. Furthermore, we assume $S_{\varepsilon}$ satisfies the following properties:

1. for every $\xi_{1}$ and $\xi_{2}$ from $\mathcal{O}\left(B_{\varepsilon}\right)$, the difference $S_{\varepsilon} \xi_{1}-S_{\varepsilon} \xi_{2}$ can be represented in the form:

$$
S_{\varepsilon} \xi_{1}-S_{\varepsilon} \xi_{2}=v_{\varepsilon}+w_{\varepsilon}, \quad \text { with } \quad\left\|v_{\varepsilon}\right\|_{\varepsilon} \leq \frac{1}{2}\left\|\xi_{1}-\xi_{2}\right\|_{\mathcal{E}}, \quad\left\|w_{\varepsilon}\right\|_{\mathcal{E}_{\varepsilon}^{1}} \leq K\left\|\xi_{1}-\xi_{2}\right\|_{\mathcal{E}},
$$

for $K>0$ independent of $\varepsilon$.

2. Furthermore, there exists a Banach space $\mathcal{E}^{-1} \supset \mathcal{\varepsilon}$ such that

$$
\|\xi\|_{\mathcal{E}^{-1}} \leq c_{-1}\|\xi\|_{\mathcal{E}}, \quad \forall \xi \in \mathcal{E} ; \quad\left\|S_{0} \xi_{1}-S_{0} \xi_{2}\right\|_{\mathcal{E}^{-1}} \leq L\left\|\xi_{1}-\xi_{2}\right\|_{\mathcal{E}^{-1}}, \quad \forall \xi_{1} \in \mathcal{O}\left(B_{\varepsilon}\right), \forall \xi_{2} \in \mathcal{O}\left(B_{0}\right),
$$

for constants $c_{-1}$ and $L>0$.

Then, for every $\varepsilon \geq 0$, the discrete dynamical system $\left(B_{\varepsilon}, S_{\varepsilon}\right)$ possesses an exponential attractor $\mathcal{M}^{\varepsilon} \subset \mathcal{O}\left(B_{\varepsilon}\right)$. The exponent of attraction $\sigma>0$ is independent of $\varepsilon \geq 0$ and $\operatorname{dim}_{f}\left(\mathcal{M}^{\varepsilon}, \varepsilon\right) \leq D$ for some positive $D$ independent of $\varepsilon$ (see Definition 6.1). Moreover

$$
\begin{aligned}
\operatorname{dist}_{\mathcal{E}^{-1}}^{S}\left(\mathcal{M}^{\varepsilon}, \mathcal{M}^{0}\right) \leq C & \left(\sup _{\xi \in \mathcal{O}\left(B_{\varepsilon}\right)}\left\|S_{\varepsilon} \xi-S_{0} \xi\right\|_{\mathcal{E}^{-1}}+\operatorname{dist}_{\mathcal{E}^{-1}}^{S}\left(B_{\varepsilon}, B_{0}\right)\right. \\
& \left.\operatorname{dist}_{\mathcal{E}^{-1}}^{S}\left(\mathcal{U}_{\varepsilon}\left(\frac{1}{4 R}, 1\right), \mathcal{U}_{0}\left(\frac{1}{4 R}, 1\right)\right)+\operatorname{dist}_{\mathcal{E}^{-1}}^{S}\left(\mathcal{U}_{\mathcal{E}}\left(\frac{1}{R}, R\right), \mathcal{U}_{0}\left(\frac{1}{R}, R\right)\right)\right)^{\varkappa},
\end{aligned}
$$

where the constants $C>0$ and $\varkappa=\varkappa\left(c_{0}, L, K, \delta_{1}\right)$ are independent of $\varepsilon$.

The proof of Theorem 6.2 is postponed to Appendix B.

We now move on to the proof of Theorem 6.1. As in the usual way, we first construct exponential attractors for the discrete dynamical systems with maps $S_{\varepsilon}:=S_{\varepsilon}(T), S_{0}:=S_{0}(T)$, for large enough $T>0$. Then by a standard procedure, clarified below, one arrives at exponential attractors for the continuous dynamical systems $\left(\mathcal{E}, S_{\varepsilon}(t)\right), t \geq 0$.

Proof of Theorem 6.1.

Step 1: Construction of discrete exponential attractors. Recall the maps $A_{\varepsilon}$ and $A_{0}$ given by (4.1). Let $\mathcal{E}=$ $H_{0}^{1}(\Omega) \times L^{2}(\Omega), \mathcal{E}^{-1}=L^{2}(\Omega) \times H^{-1}(\Omega)$, and let $\varepsilon_{\varepsilon}^{1}$ and $\varepsilon_{0}^{1}$ be given by (1.7) for $a(\cdot)=a(\dot{\bar{\varepsilon}})$ and $a(\cdot)=a^{h}$ respectively). Then property $(i)$ is an immediate consequence of the uniform ellipticity of $a(\cdot)$ and Poincarés inequality.

Proof of (ii). We shall provide an explicit construction for the covers. Moreover, it will be important later that we produce a cover such that

$$
\mathcal{U}_{\varepsilon}(\mu, r) \subset \mathcal{E}_{\varepsilon}^{2} \cap B_{\mathcal{E}_{\varepsilon}^{1}}\left(0, \delta_{r}\right), \quad \& \quad \operatorname{dist}_{\mathcal{E}^{-1}}^{S}\left(\mathcal{U}_{\varepsilon}(\mu, r), \mathcal{U}_{0}(\mu, r)\right) \leq C_{r}\left\|A_{\varepsilon}^{-1}-A_{0}^{-1}\right\|_{\mathcal{L}\left(L^{2}(\Omega)\right)},
$$

for some $C_{r}>0$ independent of $\varepsilon \geq 0$.

For this reason we seek a cover of $B_{\mathcal{E}_{\varepsilon}^{1}}(0, r)$ in the form

$$
\bigcup_{i=1}^{N(\mu, r)} B_{\varepsilon}\left(\xi_{i \varepsilon}, \mu\right), \quad \text { for } \xi_{i \varepsilon}=\left(A_{\varepsilon}^{-1}\left(p_{i}+g\right), q_{i \varepsilon}\right) \in \mathcal{E}_{\varepsilon}^{2} .
$$

To ensure $\xi_{i \varepsilon}$ are in $\varepsilon_{\varepsilon}^{2}$ we see that $\left(p_{i}, q_{i \varepsilon}\right)$ should belong to $\left(H_{0}^{1}(\Omega)\right)^{2}$ with $A_{\varepsilon} q_{i \varepsilon} \in L^{2}(\Omega)$. 
We now proceed with the construction of such a cover. As $L^{2}(\Omega) \times H_{0}^{1}(\Omega)$ is compactly embedded in $H^{-1}(\Omega) \times L^{2}(\Omega)$ then, for each $\hat{\mu}>0$, there exist finitely many $\left(p_{i}, q_{i 0}\right), i=1, \ldots, N(\hat{\mu}, r)$, such that

$$
B_{L^{2}(\Omega) \times H_{0}^{1}(\Omega)}((-g, 0), r) \subset \bigcup_{i=1}^{N(\hat{\mu}, r)} B_{H^{-1}(\Omega) \times L^{2}(\Omega)}\left(\left(p_{i}, q_{i 0}\right), \hat{\mu}\right), \quad\left(p_{i}, q_{i 0}\right) \in B_{L^{2}(\Omega) \times H_{0}^{1}(\Omega)}((-g, 0), r) .
$$

Additionally, due to density arguments, we can suppose

$$
\left(p_{i}, q_{i 0}\right) \in H_{0}^{1}(\Omega) \times H^{2}(\Omega) .
$$

Moreover, as the eigenfunctions of $A_{\varepsilon}$ form an orthonormal basis for $L^{2}(\Omega)$ we can find $q_{i \varepsilon}$ such that $A_{\varepsilon} q_{i \varepsilon} \in$ $L^{2}(\Omega)$ and

$$
\left\|q_{i \varepsilon}-q_{i 0}\right\| \leq \min \left\{\hat{\mu},\left\|A_{\varepsilon}^{-1}-A_{0}^{-1}\right\|_{\mathcal{L}\left(L^{2}(\Omega)\right)}\right\}, \quad i=1, \ldots, N(\hat{\mu}, r) .
$$

Therefore, we have the covering

$$
B_{L^{2}(\Omega) \times H_{0}^{1}(\Omega)}((-g, 0), r) \subset \bigcup_{i=1}^{N(\hat{\mu}, r)} B_{H^{-1}(\Omega) \times L^{2}(\Omega)}\left(\left(p_{i}, q_{i \varepsilon}\right), 2 \hat{\mu}\right), \quad \varepsilon \geq 0 .
$$

Now, for fixed $\xi \in B_{\mathcal{E}_{\varepsilon}^{1}}(0, r)$ we readily deduce from the ellipticity of $a$ that

$$
\left\|\nabla\left(\xi^{1}-A_{\varepsilon}^{-1}\left(p_{i}+g\right)\right)\right\| \leq v^{-1}\left\|A_{\varepsilon} \xi^{1}-p_{i}-g\right\|_{H^{-1}(\Omega)} .
$$

Furthermore, it is clear that $\left(A_{\varepsilon} \xi^{1}-g, \xi^{2}\right) \in B_{L^{2}(\Omega) \times H_{0}^{1}(\Omega)}((-g, 0), r)$. Consequently, one can readily check that

$$
B_{\mathcal{E}_{\varepsilon}^{1}}(0, r) \subset \bigcup_{i=1}^{N(\hat{\mu}, r)} B_{\mathcal{E}}\left(\left(A_{\varepsilon}^{-1}\left(p_{i}+g\right), q_{i \varepsilon}\right), 2\left(1 \vee v^{-1}\right) \hat{\mu}\right) .
$$

Additionally, since $q_{i \varepsilon}$ are obtained by truncating $q_{i 0}$ with respect to the eigenfunctions of $A_{\varepsilon}$, we compute

$$
\left\|\nabla q_{i \varepsilon}\right\|^{2} \leq v^{-1}\left(A_{\varepsilon} q_{i \varepsilon}, q_{i \varepsilon}\right) \leq v^{-1}\left(A_{\varepsilon} q_{i 0}, q_{i 0}\right) \leq v^{-2}\left\|\nabla q_{i 0}\right\|^{2},
$$

and so we deduce that

$$
\left(A_{\varepsilon}^{-1}\left(p_{i}+g\right), q_{i \varepsilon}\right) \in B_{\mathcal{\varepsilon}_{\varepsilon}^{1}}\left(0,\left(1 \vee v^{-1}\right) r\right) .
$$

Hence, upon setting $\hat{\mu}=\frac{1}{2\left(1 \vee v^{-1}\right)} \mu$, we see that the centers

$$
\mathcal{U}_{\varepsilon}(\mu, r):=\left\{\left(A_{\varepsilon}^{-1}\left(p_{i}+g\right), q_{i \varepsilon}\right) \mid i=1, \ldots, N\left(\frac{1}{2\left(1 \vee v^{-1}\right)} \mu, r\right)\right\}, \quad \varepsilon \geq 0,
$$

satisfy (ii) for $\delta_{r}=\left(1 \vee v^{-1}\right) r$. Also the additional desired properties (6.3) hold.

Construction of $B_{\varepsilon}$ and $S_{\varepsilon}$. We set $B_{\varepsilon}:=B_{\mathcal{E}_{\varepsilon}^{2}}\left(0, R_{2}\right)$ to be the absorbing ball provided by Theorem 2.5 for $\varepsilon^{2}=\mathcal{E}_{\varepsilon}^{2}$, and $a(\cdot)=a(\dot{\bar{\varepsilon}})$ in the case $\varepsilon>0$ and $a(\cdot) \equiv a^{h}$ for $\varepsilon=0$. The radius $R_{2}$ is independent of $\varepsilon$ and clearly $B_{\varepsilon}$ is closed in $\mathcal{E}$.

Since $B_{\varepsilon}$ is an absorbing set in $\varepsilon_{\varepsilon}^{2}$ and, by (6.3), $\mathcal{O}\left(B_{\varepsilon}\right)$ is a subset of $\varepsilon_{\varepsilon}^{2}$, we can choose $T_{1}$ large enough (and independent of $\varepsilon$ ) such that $S_{\varepsilon}:=S_{\varepsilon}(T), \varepsilon \geq 0$, satisfies

$$
S_{\varepsilon}: \mathcal{O}\left(B_{\varepsilon}\right) \rightarrow B_{\varepsilon}, \quad \mathcal{O}\left(B_{\varepsilon}\right)=\left(B_{\varepsilon}+\bigcup_{r \in[0,1} r \mathfrak{U}_{\varepsilon}\left(\frac{1}{4 K}, 1\right)\right) \bigcup \mathfrak{U}_{\varepsilon}\left(\frac{1}{K}, R\right) .
$$

Let us verify properties (1) and (2) of $S_{\varepsilon}$.

Proof of (1). For $\xi_{i} \in \mathcal{O}\left(B_{\varepsilon}\right) \subset \mathcal{E}_{\varepsilon}^{2}, i=1,2$, let $\xi_{u_{i}}(t)=S_{\varepsilon}(t) \xi_{i}$. Consider the splitting $u_{i}=v_{i}+w_{i}$ given by (2.2)-(2.3), and set $v=v_{1}-v_{2}$ and $w=w_{1}-w_{2}$.

As the equation for $v$ is linear then obviously the inequality

$$
\left\|v\left(T_{2}\right)\right\|_{\mathcal{E}} \leq \frac{1}{2}\left\|\xi_{1}-\xi_{2}\right\|_{\mathcal{E}}
$$


holds for large enough time $T_{2}$ (independent of $\varepsilon$ ).

From (2.3) we find that $w$ solves

$$
\left\{\begin{array}{l}
\partial_{t}^{2} w+y \partial_{t} w-\operatorname{div}(a \nabla w)=f\left(u_{2}\right)-f\left(u_{1}\right), \quad x \in \Omega, t \geq 0, \\
\left.\xi_{w}\right|_{t=0}=(0,0),\left.\quad w\right|_{\partial \Omega}=0,
\end{array}\right.
$$

for $a=a(\dot{\bar{\varepsilon}})$ or $a \equiv a^{h}$. Moreover, $p=\partial_{t} w$ solves

$$
\left\{\begin{array}{l}
\partial_{t}^{2} p+y \partial_{t} p-\operatorname{div}(a \nabla p)=f^{\prime}\left(u_{2}\right) \partial_{t} u_{2}-f^{\prime}\left(u_{1}\right) \partial_{t} u_{1}, \quad x \in \Omega, t \geq 0, \\
\left.\xi_{p}\right|_{t=0}=\left(0, f\left(\xi_{2}^{1}\right)-f\left(\xi_{1}^{1}\right)\right),\left.\quad p\right|_{\partial \Omega}=0 .
\end{array}\right.
$$

Using the fact that our initial data is from $\varepsilon_{\varepsilon}^{2}$ we conclude that $u_{i}, \partial_{t} u_{i}$ are bounded in $L^{\infty}(\Omega)$ uniformly in $\varepsilon$. Then upon testing the first equation in (6.7) with $\partial_{t} p$, rewriting the subsequent right-hand-side in the form

$$
\left(f^{\prime}\left(u_{2}\right)\left(\partial_{t} u_{2}-\partial_{t} u_{1}\right), \partial_{t} p\right)+\left(\left(f^{\prime}\left(u_{2}\right)-f^{\prime}\left(u_{1}\right)\right) \partial_{t} u_{1}, \partial_{t} p\right),
$$

we obtain via standard arguments, and the Lipschitz continuity of $S_{\varepsilon}(t)$ in $\varepsilon$ (Corollary 1.1), the uniform estimate

$$
\left\|\partial_{t} p(t)\right\|+\|\nabla p(t)\| \leq M e^{K t}\left\|\xi_{1}-\xi_{2}\right\|_{\mathcal{E}}, \quad t \geq 0 .
$$

Consequently, we use $p=\partial_{t} w$ and (6.6) to conclude

$$
\left\|\xi_{w}(t)\right\|_{\mathcal{E}_{\varepsilon}^{1}} \leq M e^{K t}\left\|\xi_{1}-\xi_{2}\right\|_{\varepsilon}, \quad t \geq 0,
$$

for some positive constants $M$ and $K$ independent of $\varepsilon$ and $\xi_{i}$. Therefore, for $T=\max \left\{T_{1}, T_{2}\right\}$, property (1) holds.

Proof of (2). This property is given by Corollary 1.2 for $a \equiv a^{h}$.

Hence, the assumptions of Theorem 6.2 hold and therefore Theorem 6.1 holds for the discrete dynamical systems $\left(B_{\varepsilon}, S_{\varepsilon}(T)\right)$ with discrete exponential attractors $\mathcal{M}_{d}^{\varepsilon}$. Indeed, Theorem 6.1 (1)-(4) hold due to the choice of $B_{\varepsilon}$ and $\mathcal{U}_{\varepsilon}$, and (5) follows from (6.2), (6.3), Theorem 4.2, Lemma 5.1 and the fact that the map $\Pi_{\varepsilon}: B_{\varepsilon} \rightarrow B_{0}$ is a bijection.

Step 2: Discrete to continuous dynamics. From the discrete exponential attractors $\mathcal{M}_{d}^{\varepsilon}$ we can build exponential attractors $\mathcal{M}^{\varepsilon}$ for the original dynamical systems $\left(\mathcal{E}, S_{\varepsilon}(t)\right)$ by the following standard construction ([5]):

$$
\mathcal{M}^{\varepsilon}:=\bigcup_{\tau \in[0, T]} S_{\varepsilon}(\tau) \mathcal{M}_{d}^{\varepsilon}, \quad \varepsilon \geq 0 .
$$

Indeed, the properties (1)-(4) can be easily verified due to dissipative estimate in $\mathcal{E}_{\varepsilon}^{2}$, Lipschitz continuity with respect to initial data in $\mathcal{E}$ (Corollary 1.1) on the bounded set $B_{\varepsilon}$ :

$$
\left\|S_{\varepsilon}(t) \xi_{1}-S_{\varepsilon}(t) \xi_{2}\right\|_{\varepsilon} \leq M\left\|\xi_{1}-\xi_{2}\right\|_{\varepsilon}, \quad \xi_{1}, \xi_{2} \in B_{\varepsilon}, \quad \varepsilon \geq 0,
$$

and Lipschitz continuity with respect to time:

$$
\left\|S_{\varepsilon}\left(\tau_{1}\right) \xi-S_{\varepsilon}\left(\tau_{2}\right) \xi\right\|_{\varepsilon} \leq M\left|\tau_{1}-\tau_{2}\right|, \quad \tau_{1}, \tau_{2} \in[0, T], \xi \in B_{\varepsilon}, \quad \varepsilon \geq 0,
$$

for some constant $M>0$ (independent of $\varepsilon$ ). Indeed, the continuity in time follows from the uniform boundedness of $B_{\varepsilon}$ in the space $\varepsilon_{\varepsilon}^{1}$. It remains to check the continuity property (5) for the exponential attractors $\mathcal{M}^{\varepsilon}$. This readily follows from the fact that (5) holds for the discrete exponential attractors $\mathcal{M}_{d}^{\varepsilon}$, Theorem 4.2 and the following computation:

$$
\begin{aligned}
\operatorname{dist}_{\mathcal{E}^{-1}}^{s}\left(\mathcal{M}^{\varepsilon}, \mathcal{M}^{0}\right) & =\operatorname{dist}_{\mathcal{E}^{-1}}^{s}\left(\bigcup_{\tau \in[0, T]} S_{\varepsilon}(\tau) \mathcal{M}_{d}^{\varepsilon}, \bigcup_{\tau \in[0, T]} S_{0}(\tau) \mathcal{M}_{d}^{0}\right) \\
& \leq \sup _{\tau \in[0, T]} \operatorname{dist}_{\mathcal{E}^{-1}}^{s}\left(S_{\varepsilon}(\tau) \mathcal{M}_{d}^{\varepsilon}, S_{0}(\tau) \mathcal{M}_{d}^{0}\right) \\
& \leq \sup _{\tau \in[0, T]} \operatorname{dist}_{\mathcal{E}^{-1}}^{s}\left(S_{\varepsilon}(\tau) \mathcal{M}_{d}^{\varepsilon}, S_{0}(\tau) \mathcal{M}_{d}^{\varepsilon}\right)+\sup _{\tau \in[0, T]} \operatorname{dist}_{\mathcal{E}^{-1}}^{s}\left(S_{0}(\tau) \mathcal{M}_{d}^{\varepsilon}, S_{0}(\tau) \mathcal{M} \mathcal{M}_{d}^{0}\right) \\
& \leq \sup _{\tau \in[0, T]} M e^{K \tau}\left\|A_{\varepsilon}^{-1}-A_{0}^{-1}\right\|_{\mathcal{L}\left(L^{2}(\Omega)\right)}+\sup _{\tau \in[0, T]} L \operatorname{dist}_{\mathcal{E}^{-1}}^{s}\left(\mathcal{M}_{d}^{\varepsilon}, \mathcal{M} \mathcal{M}_{d}^{0}\right) .
\end{aligned}
$$




\subsection{Continuity of exponential attractors in $\mathcal{E}$.}

Theorem 6.1(5) demonstrates Hölder continuity between the exponential attractors $\mathcal{M}^{\varepsilon}$ and $\mathcal{M}^{0}$ in the space $\varepsilon^{-1}$. In this section we provide continuity results in the energy space $\varepsilon$. Unlike in $\mathcal{E}^{-1}$, in the stronger topology of $\mathcal{E}$ this requires a correction (such as in Definition 5.16) of the exponential attractor $\mathcal{M}^{0}$. More precisely, the main result of this section is the following theorem.

Theorem 6.3. Assume (H1) and let $\mathcal{M}^{\varepsilon}, \mathcal{M}^{0}$ be the exponential attractors constructed in Theorem 6.1. Then, the following estimate is valid:

$$
\operatorname{dist}_{\mathcal{E}}^{s}\left(\mathcal{M}^{\varepsilon}, T_{\varepsilon} \mathcal{M}^{0}\right) \leq M \sqrt{\varepsilon}^{\varkappa}, \quad \varepsilon>0,
$$

where the 'correction' operator $T_{\varepsilon}$ is given by (5.16), $0<\varkappa<1$ as in Theorem 6.1 and the constant $M>0$ is independent of $\varepsilon$.

To prove this result, we make an important development of Theorem 6.2 to provide estimates between exponential attractors which admit correction. That is we establish the following new result.

Theorem 6.4. Let assumptions of Theorem 6.2 be satisfied and $\mathcal{M}^{\varepsilon}, \mathcal{M}^{0}$ be the exponential attractors constructed therein. Additionally, assume that:

3. for every $\varepsilon>0$ there exists a bijection $\Pi_{\varepsilon}: \varepsilon_{\varepsilon}^{1} \rightarrow \varepsilon_{0}^{1}$ that satisfies

$$
\Pi_{\varepsilon} B_{\varepsilon}=B_{0}
$$

4. for every $\varepsilon>0$ there exists a 'correction' operator $T_{\varepsilon}: \varepsilon_{0}^{1} \rightarrow \varepsilon$ which possesses the property

$$
\left\|T_{\varepsilon} \xi_{1}-T_{\varepsilon} \xi_{2}\right\|_{\varepsilon} \leq L_{\text {cor }}\left\|\xi_{1}-\xi_{2}\right\|_{\varepsilon}+m(\varepsilon) \quad \text { for all } \xi_{1}, \xi_{2} \in \mathcal{O}\left(B_{0}\right)
$$

for some constant $L_{\text {cor }}>0$ independent of $\varepsilon$ and positive function $m(\cdot)$ with $m\left(0^{+}\right)=0$.

5. the maps $S_{\varepsilon}$ are uniformly Lipschitz continuous in $\mathcal{E}$ with respect to $\varepsilon>0$, that is

$$
\left\|S_{\varepsilon} \xi_{1}-S_{\varepsilon} \xi_{2}\right\|_{\mathcal{E}} \leq L\left\|\xi_{1}-\xi_{2}\right\|_{\mathcal{E}}, \quad \forall \xi_{1}, \xi_{2} \in \mathcal{O}\left(B_{\varepsilon}\right),
$$

with some constant $L>1$ independent of $\varepsilon>0$.

Then the following estimate

$$
\begin{aligned}
\operatorname{dist}_{\mathcal{E}}^{s}\left(\mathcal{M}^{\varepsilon}, T_{\varepsilon} \mathcal{M}{ }^{0}\right) \leq C & \left(\sup _{\xi \in \mathcal{O}\left(B_{0}\right)}\left\|S_{\varepsilon} \Pi_{\varepsilon}^{-1} \xi-T_{\varepsilon} S_{0} \xi\right\|_{\mathcal{E}}+\sup _{\xi \in \mathcal{O}\left(B_{0}\right)}\left\|T_{\varepsilon} \xi-\Pi_{\varepsilon}^{-1} \xi\right\|_{\mathcal{E}}+m(\varepsilon)\right. \\
& \left.\operatorname{dist}_{\mathcal{E}}^{S}\left(\mathcal{U}_{\varepsilon}\left(\frac{1}{4 K}, 1\right), T_{\varepsilon} \mathcal{U}_{0}\left(\frac{1}{4 K}, 1\right)\right)+\operatorname{dist}_{\mathcal{E}}^{S}\left(\mathcal{U}_{\varepsilon}\left(\frac{1}{K}, R\right), T_{\varepsilon} \mathcal{U}_{0}\left(\frac{1}{K}, R\right)\right)\right)^{\varkappa},
\end{aligned}
$$

holds for constant $C>0$ independent of $\varepsilon$ and $\varkappa$ as in Theorem 6.2.

The proof of this result is presented in Appendix C.

Proof of Theorem 6.3. Let the sets $B_{\varepsilon}, \mathcal{O}\left(B_{\varepsilon}\right), \varepsilon \geq 0$, and the operator $S_{\varepsilon}=S_{\varepsilon}(T)$ be as in Theorem 6.1.

We first establish, based on the abstract result Theorem 6.4, the estimate (6.9) for the discrete exponential attractors $\mathcal{M}_{d}^{\varepsilon}$ (defined in the proof of Theorem 6.1). That is we prove the following inequality:

$$
\operatorname{dist}_{\mathcal{E}}^{S}\left(\mathcal{M}_{d}^{\varepsilon}, \mathrm{T}_{\varepsilon} \mathcal{M}{ }_{d}^{0}\right) \leq M \sqrt{\varepsilon}^{\varkappa}, \quad \varepsilon>0,
$$

for some constant $M>0$. 
Let us check that the assumptions of Theorem 6.4 hold. Indeed, assumption (3) follows from the fact that $B_{\varepsilon}=B_{\varepsilon_{\varepsilon}^{2}}\left(0, R_{2}\right)$ (see the proof of Theorem 6.1) and Definition 5.4 of the projector $\Pi_{\varepsilon}$ (where we note that $\Pi_{\varepsilon}$ can be trivially extended to the map from $\varepsilon_{\varepsilon}^{1}$ onto $\varepsilon_{0}^{1}$, preserving the bijection property). Assumption (4) holds with $m(\varepsilon)=C \varepsilon$ (for some constant $C>0$, independent of $\varepsilon$ ) due to the multiplier estimate (5.17) and the fact that $\mathcal{O}\left(B_{0}\right)$ is a bounded subset of $\varepsilon_{0}^{2}$ by construction. Assumption (5) is a consequence of Corollary 1.1. Hence the assumptions of Theorem 6.4 hold and (6.10) holds for the discrete exponential attractors $\mathcal{M}_{d}^{\varepsilon}$ and $\mathcal{M}_{d}^{0}$.

Let us now estimate the terms on the right-hand side of (6.10) in terms of $\varepsilon$. Since $\Pi_{\varepsilon}: \mathcal{E}_{\varepsilon}^{2} \rightarrow \varepsilon_{0}^{2}$ is bijective and preserves the norm (Lemma 5.1), and since $\mathcal{O}\left(B_{0}\right) \subset \mathcal{E}_{0}^{2}$ is bounded, we see that $\left\|\Pi_{\varepsilon}^{-1} \mathcal{O}\left(B_{0}\right)\right\|_{\mathcal{E}_{\varepsilon}^{2}}=$ $\left\|\mathcal{O}\left(B_{0}\right)\right\|_{\mathcal{E}_{0}^{2}}$; that is the set $\Pi_{\varepsilon}^{-1} \mathcal{O}\left(B_{0}\right)$ is bounded in $\mathcal{E}_{\varepsilon}^{2}$. Therefore, this observation and Corollary 5.2 imply that

$$
\sup _{\xi \in \mathcal{O}\left(B_{0}\right)}\left\|S_{\varepsilon} \Pi_{\varepsilon}^{-1} \xi-\mathrm{T}_{\varepsilon} S_{0} \xi\right\|_{\mathcal{E}}=\sup _{\xi \in \Pi_{\varepsilon}^{-1} \mathcal{O}\left(B_{0}\right)}\left\|S_{\varepsilon} \xi-\mathrm{T}_{\varepsilon} S_{0} \Pi_{\varepsilon} \xi\right\|_{\mathcal{E}} \leq M \sqrt{\varepsilon},
$$

for some $M>0$ independent of $\varepsilon>0$. Also from the identity

$$
\mathcal{T}_{\varepsilon} A_{0}^{-1} A_{\varepsilon} w-w=\left(\mathcal{T}_{\varepsilon} A_{0}^{-1}-A_{\varepsilon}^{-1}\right) A_{\varepsilon} w,
$$

and Remark 5.1 we deduce that

$$
\sup _{\xi \in \mathcal{O}\left(B_{0}\right)}\left\|\mathrm{T}_{\varepsilon} \xi-\Pi_{\varepsilon}^{-1} \xi\right\|_{\mathcal{\varepsilon}}=\sup _{\xi \in \Pi_{\varepsilon}^{-1} \mathcal{O}\left(B_{0}\right)}\left\|\mathrm{T}_{\varepsilon} \Pi_{\varepsilon} \xi-\xi\right\|_{\mathcal{E}} \leq M \sqrt{\varepsilon},
$$

for some constant $M>0$ independent of $\varepsilon>0$. It remains to compare the distance between the covers present in the right-hand side of (6.10). To this end, we notice that if $\xi_{i \varepsilon}:=\left(A_{\varepsilon}^{-1}\left(p_{i}+g\right), q_{i \varepsilon}\right) \in \mathcal{U}_{\varepsilon}(\mu, r)$, then

$$
\xi_{i \varepsilon}-\mathrm{T}_{\varepsilon} \xi_{i 0}=\left(\left(A_{\varepsilon}^{-1}-\mathcal{T}_{\varepsilon} A_{0}^{-1}\right)\left(p_{i}+g\right), q_{i \varepsilon}-q_{i 0}\right), \quad \varepsilon>0 .
$$

Consequently, due to Remark 5.1 and the properties of $q_{i \varepsilon}$ (see (6.4)) one can see that

$$
\operatorname{dist}_{\mathcal{E}}^{s}\left(\mathcal{U}_{\varepsilon}(\mu, r), \mathrm{T}_{\varepsilon} \mathcal{U}_{0}(\mu, r)\right) \leq C_{r} \sqrt{\varepsilon},
$$

for some constant $C_{r}>0$ independent of $\varepsilon, \mu$. Upon collecting the above estimates we derive (6.11).

It remains to establish (6.9) for the exponential attractors $\mathcal{M}^{\varepsilon}$. It is sufficient to show that

$$
\begin{aligned}
\operatorname{dist}_{\mathcal{E}}^{S}\left(\mathcal{M}^{\varepsilon}, \mathrm{T}_{\varepsilon} \mathcal{M}^{0}\right) \leq L \operatorname{dist}_{\mathcal{E}}^{\mathcal{S}}\left(\mathcal{M}_{d}^{\varepsilon}, \mathrm{T}_{\mathcal{E}} \mathcal{M}_{d}^{0}\right)+ & L \sup _{\xi \in \Pi_{\varepsilon}^{-1} \mathcal{O}\left(B_{0}\right)}\left\|\mathrm{T}_{\varepsilon} \Pi_{\varepsilon} \xi-\xi\right\|_{\mathcal{E}^{+}} \\
& +\sup _{\tau \in[0, T]} \sup _{\xi \in \Pi_{\varepsilon}^{-1} \mathcal{O}\left(B_{0}\right)}\left\|S_{\varepsilon}(\tau) \xi-\mathrm{T}_{\varepsilon} S_{0}(\tau) \Pi_{\varepsilon} \xi\right\|_{\mathcal{E}} .
\end{aligned}
$$

Indeed, since $\varkappa<1$, the above inequality, (6.11), (6.14) and Corollary 5.2 implies (6.9).

Let us demonstrate (6.17):

$$
\begin{aligned}
& \operatorname{dist}_{\mathcal{E}}^{s}\left(\mathcal{M}^{\varepsilon}, \mathrm{T}_{\varepsilon} \mathcal{M}^{0}\right)=\operatorname{dist}_{\varepsilon}^{s}\left(\bigcup_{\tau \in[0, T]} S_{\varepsilon}(\tau) \mathcal{M}_{d}^{\varepsilon}, \bigcup_{\tau \in[0, T]} \mathrm{T}_{\varepsilon} S_{0}(\tau) \mathcal{M}_{d}^{0}\right) \\
& \leq \sup _{\tau \in[0, T]} \operatorname{dist}_{\varepsilon}^{s}\left(S_{\varepsilon}(\tau) \mathcal{M}_{d}^{\varepsilon}, \mathrm{T}_{\varepsilon} S_{0}(\tau) \mathcal{M} \mathcal{M}_{d}^{0}\right) \\
& \leq \sup _{\tau \in[0, T]} \operatorname{dist}_{\mathcal{E}}^{s}\left(S_{\varepsilon}(\tau) \mathcal{M}_{d}^{\varepsilon}, S_{\varepsilon}(\tau) \Pi_{\varepsilon}^{-1} \mathcal{M} \mathcal{M}_{d}^{0}\right)+\sup _{\tau \in[0, T]} \operatorname{dist}_{\mathcal{E}}^{s}\left(S_{\varepsilon}(\tau) \Pi_{\varepsilon}^{-1} \mathcal{M}_{d}^{0}, \mathrm{~T}_{\varepsilon} S_{0}(\tau) \mathcal{M}_{d}^{0}\right) \\
& \leq L \operatorname{dist}_{\mathcal{E}}^{s}\left(\mathcal{M}_{d}^{\varepsilon}, \Pi_{\varepsilon}^{-1} \mathcal{M}_{d}^{0}\right)+\sup _{\tau \in[0, T]} \operatorname{dist}_{\mathcal{E}}^{s}\left(S_{\varepsilon}(\tau) \Pi_{\varepsilon}^{-1} \mathcal{M}_{d}^{0}, \mathrm{~T}_{\varepsilon} S_{0}(\tau) \mathcal{M}_{d}^{0}\right) \\
& \leq L \operatorname{dist}_{\mathcal{E}}^{s}\left(\mathcal{M}_{d}^{\varepsilon}, \mathrm{T}_{\varepsilon} \mathcal{M}_{d}^{0}\right)+L \operatorname{dist}_{\varepsilon}^{s}\left(\mathrm{~T}_{\varepsilon} \mathcal{M}_{d}^{0}, \Pi_{\varepsilon}^{-1} \mathcal{M}_{d}^{0}\right)+\sup _{\tau \in[0, T]} \operatorname{dist}_{\varepsilon}^{s}\left(S_{\varepsilon}(\tau) \Pi_{\varepsilon}^{-1} \mathcal{M} \mathbb{d}_{d}^{0}, \mathrm{~T}_{\varepsilon} S_{0}(\tau) \mathcal{M} \mathcal{M}_{d}^{0}\right) \\
& \leq L \operatorname{dist}_{\mathcal{E}}^{s}\left(\mathcal{M}_{d}^{\varepsilon}, \mathrm{T}_{\varepsilon} \mathcal{M}_{d}^{0}\right)+L \sup _{\xi \in \mathcal{O}\left(B_{0}\right)}\left\|\mathrm{T}_{\varepsilon} \xi-\Pi_{\varepsilon}^{-1} \xi\right\|_{\varepsilon}+\sup _{\tau \in[0, T]} \sup _{\xi \in \mathcal{O}\left(B_{0}\right)}\left\|S_{\varepsilon}(\tau) \Pi_{\varepsilon}^{-1} \xi-\mathrm{T}_{\varepsilon} S_{0}(\tau) \xi\right\|_{\mathcal{E}} \\
& \leq L \operatorname{dist}_{\mathcal{E}}^{s}\left(\mathcal{M}_{d}^{\varepsilon}, \mathrm{T}_{\varepsilon} \mathcal{M}_{d}^{0}\right)+L \sup _{\xi \in \Pi_{\varepsilon}^{-1} \mathcal{O}\left(B_{0}\right)}\left\|\mathrm{T}_{\varepsilon} \Pi_{\varepsilon} \xi-\xi\right\|_{\mathcal{E}}+\sup _{\tau \in[0, T]} \sup _{\xi \in \Pi_{\varepsilon}^{-1} \mathcal{O}\left(B_{0}\right)}\left\|S_{\varepsilon}(\tau) \xi-\mathrm{T}_{\varepsilon} S_{0}(\tau) \Pi_{\varepsilon} \xi\right\|_{\mathcal{\varepsilon}} .
\end{aligned}
$$

Hence the theorem is proved. 


\section{The case of different boundary conditions}

In this section we are going to show that the analogues of the obtained homogenisation error estimates for the global and exponential attractors still hold if we change the Dirichlet boundary conditions to be either Neumann or periodic.

Let $\Omega \subset \mathbb{R}^{3}$ be a smooth bounded domain and $\mathcal{H}^{1}:=H^{1}(\Omega)$ or $\Omega$ be a three-dimensional torus $\mathbb{T}^{3}:=$ $[0, \ell)^{3}, \ell>0$, with

$$
\mathcal{H}^{1}:=\left\{u \in H^{1}(\Omega) \mid u\left(x+\ell e_{k}\right)=u(x), k \in\{1,2,3\}\right\} .
$$

In both cases we endow $\mathcal{H}^{1}$ with the norm

$$
\|u\|_{\mathcal{H}^{1}}^{2}:=\|\nabla u\|^{2}+\|u\|^{2}, \quad u \in \mathcal{H}^{1} .
$$

For the maps $A_{\varepsilon}$ be given by (4.1), $\varepsilon \geq 0$, we consider the problem

$$
\left\{\begin{array}{l}
\partial_{t}^{2} u^{\varepsilon}+y \partial_{t} u^{\varepsilon}+\left(A_{\varepsilon}+1\right) u^{\varepsilon}+f\left(u^{\varepsilon}\right)=g(x), \quad x \in \Omega, t \geq 0, \\
\left.\left(u^{\varepsilon}, \partial_{t} u^{\varepsilon}\right)\right|_{t=0}=\xi
\end{array}\right.
$$

endowed with either Neumann

$$
\left\{\begin{array}{l}
\left.a(\dot{\bar{\varepsilon}}) \nabla u^{\varepsilon} \cdot n\right|_{\partial \Omega}=0, \quad \varepsilon>0 \\
\left.a^{h} \nabla u^{0} \cdot n\right|_{\partial \Omega}=0, \quad \varepsilon=0
\end{array}\right.
$$

or periodic

$$
\left\{\begin{array}{l}
u^{\varepsilon}\left(x+\ell e_{k}\right)=u^{\varepsilon}(x), \\
\nabla u^{\varepsilon}\left(x+\ell e_{k}\right)=\nabla u^{\varepsilon}(x),
\end{array} \quad k \in\{1,2,3\}, \varepsilon \geq 0,\right.
$$

boundary conditions.

It is well-known that problem (7.1) with either boundary conditions $(\mathrm{N})$ or $(\mathrm{P})$ is well-posed in the energy space $\mathcal{E}:=\mathcal{H}^{1} \times L^{2}(\Omega)$ and, therefore, defines a dynamical system $\left(\mathcal{E}, S_{\varepsilon}(t)\right)$ where

$$
S_{\varepsilon}(t) \xi:=\xi_{u^{\varepsilon}}(t), \quad t \geq 0,
$$

for $u^{\varepsilon}(t)$ the unique solution of the corresponding problem with initial data $\xi$.

Moreover, is well-known that $A_{\varepsilon}+1: \mathcal{D}\left(A_{\varepsilon}+1\right) \subset L^{2}(\Omega) \rightarrow L^{2}(\Omega)$ is self-adjoint, where

$$
\mathcal{D}\left(A_{\varepsilon}+1\right)=\left\{\begin{array}{l}
\left\{u \in \mathcal{H}^{1}\left|A_{\varepsilon} u \in L^{2}(\Omega), a(\dot{\bar{\varepsilon}}) \nabla u \cdot n\right|_{\partial \Omega}=0\right\}, \quad \varepsilon>0, \\
\left\{u \in \mathcal{H}^{1}\left|A_{0} u \in L^{2}(\Omega), a^{h} \nabla u \cdot n\right|_{\partial \Omega}=0\right\}, \quad \varepsilon=0,
\end{array}\right.
$$

for condition $(\mathrm{N})$ or

$$
\mathcal{D}\left(A_{\varepsilon}+1\right)=\left\{u \in \mathcal{H}^{1} \mid A_{\varepsilon} u \in L^{2}(\Omega), \nabla u\left(x+\ell e_{k}\right)=\nabla u(x), k \in\{1,2,3\}\right\}, \varepsilon \geq 0,
$$

for condition (P). Setting

$$
\left\{\begin{array}{l}
\varepsilon_{\varepsilon}^{2}:=\left\{\xi \in\left(\mathcal{D}\left(A_{\varepsilon}+1\right)\right)^{2} \mid\left(A_{\varepsilon} \xi^{1}-g\right) \in \mathcal{H}^{1}\right\}, \\
\|\xi\|_{\mathcal{E}_{\varepsilon}^{2}}^{2}:=\left\|A_{\varepsilon} \xi^{1}-g\right\|_{\mathcal{H}^{1}}^{2}+\left\|\left(A_{\varepsilon}+1\right) \xi^{1}\right\|^{2}+\left\|\left(A_{\varepsilon}+1\right) \xi^{2}\right\|^{2},
\end{array} \quad \varepsilon \geq 0,\right.
$$

it is straightforward to see from Appendix A and Sections 2-6 that the following theorem holds.

Theorem 7.1. Assume (H1). Then, for every $\varepsilon \geq 0$, the dynamical systems $\left(\varepsilon, S_{\varepsilon}(t)\right)$ generated by problem (7.1) with boundary conditions $(\mathrm{N})$ or $(\mathrm{P})$ possesses a global attractor $\mathcal{A}^{\varepsilon}$, and exponential attractor $\mathcal{M}^{\varepsilon}$, of finite fractal dimension such that:

$$
\begin{aligned}
& \mathcal{A}^{\varepsilon} \subset \mathcal{M}^{\varepsilon} \subset \mathcal{E}_{\varepsilon}^{2}, \quad\left\|\mathcal{A}^{\varepsilon}\right\|_{\mathcal{E}_{\varepsilon}^{2}} \leq\left\|\mathcal{M}^{\varepsilon}\right\|_{\mathcal{E}_{\varepsilon}^{2}} \leq M(\|g\|), \quad \mathcal{A}^{\varepsilon}=\left.\mathcal{K}^{\varepsilon}\right|_{t=0}, \\
& \operatorname{dist}_{\mathcal{E}}\left(S_{\varepsilon}(t) B, \mathcal{M}^{\varepsilon}\right) \leq e^{-\sigma t} M\left(\|B\|_{\mathcal{E}}\right), \quad t \geq 0, \quad \text { for all bounded } B \subset \mathcal{E}, \\
& \operatorname{dim}_{f}\left(\mathcal{A}^{\varepsilon}, \mathcal{E}\right) \leq \operatorname{dim}_{f}\left(\mathcal{M}^{\varepsilon}, \mathcal{E}\right) \leq D,
\end{aligned}
$$

where the constants $\sigma, D>0$ and non-decreasing function $M$ are independent of $\varepsilon$. Here $\mathcal{K}^{\varepsilon}$ is the set of all bounded energy solutions to problem (7.1), with (N) or (P), defined for all $t \in \mathbb{R}$. 
Let us now discuss error estimates between the anisotropic and homogenised attractors. It is known that the main homogenisation results, Theorems 4.1 and 5.1, remain valid for the case of Neumann and periodic boundary conditions.

Theorem 7.2 ([16]). Let $\Omega \subset \mathbb{R}^{3}$ be a bounded smooth domain or three-dimensional torus $\mathbb{T}^{3}, \varepsilon>0$, periodic matrix $a(\cdot)$ satisfying uniform ellipticity and boundedness assumptions, $A_{\varepsilon}$ and $A_{0}$ given by (4.1) and $g \in L^{2}(\Omega)$. Let also $u^{\varepsilon} \in \mathcal{D}\left(A_{\varepsilon}+1\right), u^{0} \in \mathcal{D}\left(A_{0}+1\right)$, solve the equations

$$
\left(A_{\varepsilon}+1\right) u^{\varepsilon}=g \text { in } \Omega, \quad\left(A_{0}+1\right) u^{0}=g \text { in } \Omega .
$$

Then, the following estimates

$$
\begin{aligned}
& \left\|u^{\varepsilon}-u^{0}\right\| \leq C \varepsilon\|g\|, \\
& \left\|u^{\varepsilon}-\mathcal{T}_{\varepsilon} u^{0}\right\|_{\mathcal{H}^{1}} \leq C \sqrt{\varepsilon}\|g\|,
\end{aligned}
$$

hold for some constant $C=C(v, \Omega)$. Here the operator $\mathcal{T}_{\varepsilon}$ is given in (5.1).

Remark 7.1. Note that inequalities (7.3) and (7.4) are equivalent to the following operator estimates:

$$
\begin{aligned}
& \left\|\left(A_{\varepsilon}+1\right)^{-1}-\left(A_{0}+1\right)^{-1}\right\|_{\mathcal{L}\left(L^{2}(\Omega)\right)} \leq C \varepsilon, \\
& \left\|\left(A_{\varepsilon}+1\right)^{-1} g-\mathcal{T}_{\varepsilon}\left(A_{0}+1\right)^{-1} g\right\|_{\mathcal{H}^{1}} \leq C \sqrt{\varepsilon}\|g\|, \quad \forall g \in L^{2}(\Omega) .
\end{aligned}
$$

Remark 7.2. In the case of periodic boundary conditions $(\mathrm{P})$, where $Q=[0,1)^{3}$ and $\Omega=[0, \ell)^{3}$, if $\frac{\ell}{\varepsilon} \in \mathbb{N}$ then for $w \in \mathcal{D}\left(A_{0}+1\right)$ the corrector $\mathcal{T}_{\varepsilon} w$ belongs to $\mathcal{H}^{1}$. In this setting it is well-known that one can improve the bound in (7.4) from $\sqrt{\varepsilon}$ to $\varepsilon$. Consequently, as discussed in Remark 5.2, for this case we can replace $\sqrt{\varepsilon}$ with $\varepsilon$ in the relevant results below.

Let us also define the energy space of order -1 :

$$
\mathcal{E}^{-1}:=L^{2}(\Omega) \times\left(\mathcal{H}^{1}\right)^{*},
$$

where $\left(\mathcal{H}^{1}\right)^{\star}$ stands for the dual space of $\mathcal{H}^{1}$.

We now draw the reader's attention to the fact that the key theorems (Theorems 4.2 and 5.2) on the distance between trajectories in $\varepsilon^{-1}$ are in terms of resolvents of the operator $A_{\varepsilon}, \varepsilon \geq 0$. The key point to note is that the proofs of these results essentially rely on the fact $A_{\varepsilon}$ is self-adjoint and (uniformly in $\varepsilon$ ) bounded and positive. Since the operator $A_{\varepsilon}+1$, for Neumann (N) or periodic (P) boundary conditions, also possesses these properties one can see that analogues of Theorems 4.2-5.2 readily hold (after appropriately changing the projector $\Pi_{\varepsilon}$ ). Namely, upon defining $\Pi_{\varepsilon}: \varepsilon_{\varepsilon}^{2} \rightarrow \varepsilon_{0}^{2}$, for $\varepsilon_{\varepsilon}^{2}$ given by (7.2), as follows

$$
\Pi_{\varepsilon}\left(\xi^{1}, \xi^{2}\right):=\left(\xi_{0}^{1}, \xi_{0}^{2}\right), \quad \text { where }\left\{\begin{array}{l}
\text { the term } \xi_{0}^{i} \in \mathcal{D}\left(A_{0}+1\right), i=1,2, \text { satisfies } \\
\left(A_{0}+1\right) \xi_{0}^{i}=\left(A_{\varepsilon}+1\right) \xi^{i},
\end{array}\right.
$$

we have the following result.

Theorem 7.3. Let $\varepsilon_{\varepsilon}^{2}$ be given by (7.2) and $S_{\varepsilon}(t)$ be the solution operator to the problem (7.1) with Neumann $(\mathrm{N})$ or periodic (P) boundary conditions. Then, for all $\xi \in \mathcal{E}_{\varepsilon}^{2},\|\xi\|_{\mathcal{E}_{\varepsilon}^{2}} \leq R, R>0$, the inequalities

$$
\begin{aligned}
& \left\|S_{\varepsilon}(t) \xi-S_{0}(t) \xi\right\|_{\mathcal{E}^{-1}}+\left\|S_{\varepsilon}(t) \xi-S_{0}(t) \Pi_{\varepsilon} \xi\right\|_{\mathcal{E}^{-1}} \leq M e^{K t}\left\|\left(A_{\varepsilon}+1\right)^{-1}-\left(A_{0}+1\right)^{-1}\right\|_{\mathcal{L}\left(L^{2}(\Omega)\right)}, \\
& \left\|\partial_{t} S_{\varepsilon}(t) \xi-\partial_{t} S_{0}(t) \Pi_{\varepsilon} \xi\right\|_{\mathcal{E}^{-1}} \leq M e^{K t}\left\|\left(A_{\varepsilon}+1\right)^{-1}-\left(A_{0}+1\right)^{-1}\right\|_{\mathcal{L}\left(L^{2}(\Omega)\right)}^{1 / 2}, \quad t \geq 0, \\
& \left\|S_{\varepsilon}(t) \xi-T_{\varepsilon} S_{0}(t) \Pi_{\varepsilon} \xi\right\|_{\varepsilon} \leq M e^{K t} \sqrt{\varepsilon},
\end{aligned}
$$

hold for some non-decreasing functions $M=M(R,\|g\|)$ and $K=K(R,\|g\|)$ which are independent of $\varepsilon>0$.

Based on Theorem 7.3 and arguing along the same lines as in Sections 4 - 6 we obtain the following theorem on the comparison of distances between anisotropic and homogenised attractors in terms of $\varepsilon$. 
Theorem 7.4. Assume (H1) and (H2). Let $\mathcal{A}^{\varepsilon}, \mathcal{M}^{\varepsilon}, \varepsilon \geq 0$ be attractors corresponding to problem (7.1), with Neuman $(\mathrm{N})$ or periodic $(\mathrm{P})$ boundary conditions, provided by Theorem 7.1. Let also $\alpha>0$ be such an exponent that $\left(A_{\varepsilon}+1\right)^{-1} \in \mathcal{L}\left(L^{2}(\Omega), C^{\alpha}(\bar{\Omega})\right)$ and $0 \leq \beta<\alpha$. Then, the following estimates

$$
\begin{array}{lll}
\operatorname{dist}_{\mathcal{E}^{-1}}\left(\mathcal{A}^{\varepsilon}, \mathcal{A}^{0}\right) \leq M \varepsilon^{\varkappa}, & \operatorname{dist}_{\mathcal{E}}\left(\mathcal{A}^{\varepsilon}, T_{\varepsilon} \mathcal{A}^{0}\right) \leq M \sqrt{\varepsilon^{\varkappa}}, & \operatorname{dist}_{\left(C^{\beta}(\bar{\Omega})\right)^{2}}\left(\mathcal{A}^{\varepsilon}, \mathcal{A}^{0}\right) \leq M \varepsilon^{\theta \varkappa}, \\
\operatorname{dist}_{\mathcal{E}^{-1}}^{s}\left(\mathcal{M}^{\varepsilon}, \mathcal{M}^{0}\right) \leq M \varepsilon^{\varkappa}, & \operatorname{dist}_{\mathcal{E}}^{s}\left(\mathcal{M}^{\varepsilon}, T_{\mathcal{\varepsilon}} \mathcal{M}^{0}\right) \leq M \sqrt{\varepsilon^{\varkappa}}, & \operatorname{dist}_{\left(C^{\beta}(\bar{\Omega})\right)^{2}}^{s}\left(\mathcal{M}^{\varepsilon}, \mathcal{M}^{0}\right) \leq M \varepsilon^{\theta \varkappa},
\end{array}
$$

hold for some non-decreasing $M=M(\|g\|)$ and constants $\varkappa \in(0,1), \theta=\frac{\alpha-\beta}{2+\alpha}$ independent of $\varepsilon$. Here $T_{\varepsilon}$ is the 'correction' operator defined by (5.16).

\section{A Proof of Theorem 1.3}

To prove Theorem 1.3 we perform a splitting of the solution $u=v+w$ to the problem (1.2) into asymptotically contractive and compact parts. This form of splitting was intoduced in [31].

Let us consider

$$
\left\{\begin{array}{l}
\partial_{t}^{2} v+y \partial_{t} v-\operatorname{div}(a \nabla v)+L v+f(u)-f(w)=0, x \in \Omega, t \geq 0 \\
\left.\xi_{v}\right|_{t=0}=\xi_{u}(0),\left.v\right|_{\partial \Omega}=0
\end{array}\right.
$$

and

$$
\left\{\begin{array}{l}
\partial_{t}^{2} w+y \partial_{t} w-\operatorname{div}(a \nabla w)+L w+f(w)=L u+g, x \in \Omega, t \geq 0, \\
\left.\xi_{w}\right|_{t=0}=0,\left.w\right|_{\partial \Omega}=0,
\end{array}\right.
$$

where the fixed constant $L>0$ is specified below.

Recall that $\mathcal{B}$ denotes a positive invariant absorbing set of the semigroup $(\mathcal{E}, S(t))$ (see (1.6)). Similar to Theorem 1.1 we have the following result.

Lemma A.1. Assume (H1), $\xi_{u}(0) \in \mathcal{B}, L>0$ be an arbitrary constant and $w$ solve the equation (A.2). Then the estimate

$$
\left\|\xi_{w}(t)\right\|_{\mathcal{E}} \leq M_{L}\left(\|\mathcal{B}\|_{\mathcal{E}}\right), \quad t \geq 0
$$

holds for some non-decreasing function $M_{L}$ that depends only on $v$ and $L$.

The proof of Lemma A.1 follows from the multiplication of the first equation in (A.2) by $\partial_{t} w+\kappa w$ with sufficiently small $\kappa>0$ and the fact that we already know that $\left\|\xi_{u}(t)\right\|_{\mathcal{E}} \leq M\left(\|\mathcal{B}\|_{\mathcal{E}}\right.$ ) for all $t \geq 0$ (due to the dissipative estimate (1.3)).

Lemma A.2. Assume (H1), $\xi_{u}(0) \in \mathcal{B}, L>0$ be an arbitrary constant and $w$ solve (A.2). Then, for every $\mu>0$ the estimate

$$
\int_{s}^{t}\left\|\partial_{t} w(\tau)\right\|^{2} d \tau \leq \mu(t-s)+\frac{M_{L}\left(\|\mathcal{B}\|_{\mathcal{E}}\right)}{\mu}, \quad t \geq s \geq 0,
$$

holds for some non-decreasing function $M_{L}$ that depends only on $v$ and $L$.

Proof. Multiplying the equation (A.2) by $\partial_{t} w$, integrating in $\Omega$ and using Lemma A.1 we obtain

$$
\frac{d}{d t} \Lambda+y\left\|\partial_{t} w\right\|^{2}=-L\left(\partial_{t} u, w\right) \leq y \mu+\frac{y^{-1} L^{2} M_{L}\left(\|\mathcal{B}\|_{\mathcal{E}}\right)}{\mu}\left\|\partial_{t} u\right\|^{2}
$$

where

$$
\Lambda=\frac{1}{2}\left(\left\|\partial_{t} w\right\|^{2}+(a \nabla w, \nabla w)+L\|w\|^{2}\right)+(F(w), 1)-L(u, w)-(g, w) .
$$


From the dissipative estimate (1.3) and positive invariance (1.6) we see that

$$
\int_{s}^{t}\left\|\partial_{t} u(\tau)\right\|^{2} d \tau \leq M\left(\|\mathcal{B}\|_{\varepsilon}\right), \quad t \geq s \geq 0 .
$$

Integrating (A.4) in time from $s$ to $t$, using Lemma A.1 and (A.5) we derive the desired inequality (A.3) for some new function $M_{L}$.

Before continuing, let us recall the following modified Gronwall's lemma.

Lemma A.3 (Modified Gronwall's Lemma [31]). Let $\Lambda: \mathbb{R}^{+} \rightarrow \mathbb{R}^{+}$be an absolutely continuous function satisfying

$$
\frac{d}{d t} \Lambda(t)+2 \mu \Lambda(t) \leq h(t) \Lambda(t)+k,
$$

where $\mu>0, k \geq 0$ and $\int_{s}^{t} h(\tau) d \tau \leq \mu(t-s)+m$, for all $t \geq s \geq 0$ and some $m \geq 0$. Then

$$
\Lambda(t) \leq \Lambda(0) e^{m} e^{-\mu t}+\frac{k e^{m}}{\mu}, \quad t \geq 0 .
$$

We are now ready to show that $v$ exponentially goes to 0 in the energy space $\mathcal{E}$.

Proposition A.1. Assume $(\mathrm{H} 1)$ and $\xi_{u}(0) \in \mathcal{B}$. Then, for sufficiently large constant $L=L(y, v, f)$, the estimate

$$
\left\|\xi_{v}(t)\right\|_{\mathcal{E}} \leq M_{L}\left(\|\mathcal{B}\|_{\mathcal{E}}\right) e^{-\beta t}, \quad t \geq 0,
$$

holds for some non-decreasing function $M_{L}$ and constant $\beta>0$ that depend only on $v$ and $L$.

Proof. Fix $\kappa>0$ to be specified below. Multiplying equation (A.1) by $\partial_{t} v+\kappa v$ in $L^{2}(\Omega)$ we find (after some algebraic manipulation) that

$$
\begin{aligned}
\frac{d}{d t} \Lambda+(y-\kappa)\left\|\partial_{t} v\right\|^{2}+\kappa\left((a \nabla v, \nabla v)+L\|v\|^{2}+(f(u)-f(w), v)\right) & = \\
& \left(f^{\prime}(u)-f^{\prime}(w), \partial_{t} w v\right)-\frac{1}{2}\left(f^{\prime \prime}(u) \partial_{t} u,|v|^{2}\right),
\end{aligned}
$$

for

$$
\begin{aligned}
\Lambda:=\frac{1}{2}\left(\left\|\partial_{t} v\right\|^{2}+(a \nabla v, \nabla v)+L\|v\|^{2}\right)+\kappa\left(\partial_{t} v, v\right)+\frac{\kappa y}{2}\|v\|^{2}+ & \\
& (f(u)-f(w), v)-\frac{1}{2}\left(f^{\prime}(u),|v|^{2}\right) .
\end{aligned}
$$

Now by the lower bound on $f^{\prime}$ (see (H1)) we compute

$$
L\|v\|^{2}+(f(u)-f(w), v)=L\|v\|^{2}+\left(\int_{0}^{1} f^{\prime}(\lambda u+(1-\lambda) w) d \lambda,|v|^{2}\right) \geq\left(L-K_{2}\right)\|v\|^{2} .
$$

Thus, for $L>K_{2}$, (A.6) implies

$$
\frac{d}{d t} \Lambda+(y-\kappa)\left\|\partial_{t} v\right\|^{2}+\kappa(a \nabla v, \nabla v) \leq\left(f^{\prime}(u)-f^{\prime}(w), \partial_{t} w v\right)-\frac{1}{2}\left(f^{\prime \prime}(u) \partial_{t} u,|v|^{2}\right) .
$$

We shall establish below, for sufficiently large $L$, the equivalence

$$
C_{v} \Lambda \leq \frac{1}{2}\left\|\partial_{t} v\right\|^{2}+\frac{1}{2}(a \nabla v, \nabla v) \leq 2 \Lambda .
$$

as well as the inequalities

$$
\begin{aligned}
& \left(f^{\prime}(u)-f^{\prime}(w), \partial_{t} w v\right) \leq \frac{\kappa}{4}(a \nabla v, \nabla v)+M_{L}\left(\|\mathcal{B}\|_{\mathcal{E}}\right)\left\|\partial_{t} w\right\|^{2} \Lambda, \\
& -\frac{1}{2}\left(f^{\prime \prime}(u), \partial_{t} u|v|^{2}\right) \leq \frac{\kappa}{4}(a \nabla v, \nabla v)+M\left(\|\mathcal{B}\|_{\mathcal{E}}\right)\left\|\partial_{t} u\right\|^{2} \Lambda,
\end{aligned}
$$


Consequently, for $0<\kappa<y / 2$, inequalities (A.8)-(A.11) imply

$$
\frac{d}{d t} \Lambda+C_{\nu} \kappa \Lambda \leq h \Lambda, \quad \text { for } h(t)=M_{L}\left(\|\mathcal{B}\|_{\mathcal{E}}\right)\left(\left\|\partial_{t} w(t)\right\|^{2}+\left\|\partial_{t} u(t)\right\|^{2}\right) .
$$

This inequality, Lemma A.2 (with small enough $\mu$ ) and (A.5) show that the assumptions of the Modified Gronwall's Lemma (Lemma A.3) hold. Whence

$$
\Lambda(t) \leq M(\|\mathcal{B}\|) \Lambda(0) e^{-\frac{1}{2} c_{v} \kappa t}, \quad t \geq 0 .
$$

From (A.9), and the fact $\xi_{v}(0)=\xi_{u}(0)$, we prove the desired result. Therefore, to complete the proof it remains to establish (A.9)-(A.11).

Let us prove (A.9). We shall prove the upper bound, as the argument for the lower bound is similar. For $\kappa \in(0, y / 2)$, utilising the dissipative estimate for $u$ (1.3) and the bounds on $f^{\prime}$ (see (H1) and Remark 1.1.a) we compute

$$
\begin{aligned}
\Lambda & \geq \frac{1}{4}\left\|\partial_{t} v\right\|^{2}+\frac{1}{2}\left((a \nabla v, \nabla v)+L\|v\|^{2}\right)+\kappa\left(\frac{y}{2}-\kappa\right)\|v\|^{2}+\left(\int_{0}^{1} f^{\prime}(\lambda u+(1-\lambda) w) d \lambda,|v|^{2}\right)-\frac{1}{2}\left(f^{\prime}(u),|v|^{2}\right) \\
& \geq \frac{1}{4}\left\|\partial_{t} v\right\|^{2}+\frac{1}{2}\left((a \nabla v, \nabla v)+L\|v\|^{2}\right)-K_{2}\|v\|^{2}-\frac{K_{4}}{2}\left(1+|u|^{2},|v|^{2}\right) \\
& \geq \frac{1}{4}\left\|\partial_{t} v\right\|^{2}+\frac{1}{2}\left((a \nabla v, \nabla v)+L\|v\|^{2}\right)-\left(K_{2}+\frac{K_{4}}{2}\right)\|v\|^{2}-\frac{K_{4}}{2}\|u\|_{L^{4}(\Omega)}^{2}\|v\|^{1 / 2}\|v\|_{L^{6}(\Omega)}^{3 / 2} \\
& \geq \frac{1}{4}\left\|\partial_{t} v\right\|^{2}+\frac{1}{4}(a \nabla v, \nabla v)+\left(\frac{L}{2}-K_{2}-\frac{K_{4}}{2}-M\left(\|\mathcal{B}\|_{\mathcal{E}}\right)\right)\|v\|^{2} .
\end{aligned}
$$

Then for large enough $L$, we deduce $\Lambda \geq \frac{1}{4}\left\|\partial_{t} v\right\|^{2}+\frac{1}{4}(a \nabla v, \nabla v)$ and the upper bound in (A.9) holds.

To prove (A.10) and (A.11), we use dissipative bounds on $u$ and $w$ (Lemma A.1) plus the growth assumption on $f^{\prime \prime}$ to establish

$$
\begin{aligned}
\left(f^{\prime}(u)-f^{\prime}(w), \partial_{t} w v\right) & \leq K_{5}\left(1+|u|+|w|,\left|\partial_{t} w \| v\right|^{2}\right) \leq K_{5}\|1+|u|+|w|\|_{L^{6}(\Omega)}\left\|\partial_{t} w\right\|\left\|\left|\left\|\left.v\right|^{2}\right\|_{L^{3}(\Omega)}\right.\right. \\
& \leq M_{L}\left(\|\mathcal{B}\|_{\varepsilon}\right)\left\|\partial_{t} w\right\|\|\nabla v\|^{2} \leq \frac{\kappa v}{4}\|\nabla v\|^{2}+M_{L}\left(\|\mathcal{B}\|_{\mathcal{E}}\right)\left\|\partial_{t} w\right\|^{2}\|\nabla v\|^{2},
\end{aligned}
$$

and

$$
-\frac{1}{2}\left(f^{\prime \prime}(u), \partial_{t} u|v|^{2}\right) \leq M\left(\|\mathcal{B}\| \varepsilon_{\mathcal{E}}\right)\left\|\partial_{t} u\right\|\|v\|_{L^{6}(\Omega)}^{2} \leq \frac{\kappa v}{4}\|\nabla v\|^{2}+M(\|\mathcal{B}\| \varepsilon)\left\|\partial_{t} u\right\|^{2}\|\nabla v\|^{2} .
$$

Then the desired inequalities follow by invoking the ellipticity of $a$ and the now established (A.9). The proof is complete.

To complete the proof of Theorem 1.3 it remains to prove that $\xi_{w}$ is a bounded trajectory in $\mathcal{E}^{1}$, this is the subject of the next result.

Proposition A.2. Assume $(\mathrm{H} 1)$ and $\xi_{u}(0) \in \mathcal{B}$. Then, for sufficiently large constant $L=L(y, v, f)$, the inequality

$$
\|\operatorname{div}(a \nabla w)(t)\|+\left\|\nabla \partial_{t} w(t)\right\|+\left\|\partial_{t}^{2} w(t)\right\| \leq M_{L}\left(\|\mathcal{B}\|_{\mathcal{E}}\right), \quad t \geq 0,
$$

holds for some non-decreasing function $M_{L}$ that depends only on $v$ and $L$.

Proof. Let us set $q:=\partial_{t} w$, then $q$ solves

$$
\left\{\begin{array}{l}
\partial_{t}^{2} q+y \partial_{t} q-\operatorname{div}(a \nabla q)+L q+f^{\prime}(w) q=L \partial_{t} u, \quad x \in \Omega, t \geq 0, \\
\left.\xi_{q}\right|_{t=0}=(0, L u(0)+g),\left.\quad q\right|_{\partial \Omega}=0 .
\end{array}\right.
$$

Multiplying the first equation above by $\partial_{t} q+\kappa q$ and integrating in $\Omega$ we find

$$
\begin{aligned}
& \frac{d}{d t} \Lambda+(y-2 \kappa)\left\|\partial_{t} q\right\|^{2}+\kappa\left(\left\|\partial_{t} q\right\|^{2}+(a \nabla q, \nabla q)+L\|q\|^{2}+\left(f^{\prime}(w),|q|^{2}\right)\right)= \\
& L\left(\partial_{t} u, \partial_{t} q\right)+\kappa L\left(\partial_{t} u, \partial_{t} w\right)+\frac{1}{2}\left(f^{\prime \prime}(w) \partial_{t} w,|q|^{2}\right),
\end{aligned}
$$


for

$$
\Lambda:=\frac{1}{2}\left(\left\|\partial_{t} q\right\|^{2}+(a \nabla q, \nabla q)+L\|q\|^{2}+\left(f^{\prime}(w),|q|^{2}\right)\right)+\kappa\left(\partial_{t} q, q\right)+\frac{\kappa y}{2}\|q\|^{2} .
$$

The identity (A.12) can be rewritten in the form

$$
\begin{aligned}
\frac{d}{d t} \Lambda+(y-2 \kappa)\left\|\partial_{t} q\right\|^{2}+2 \kappa \Lambda & =2 \kappa^{2}\left(\partial_{t} w, \partial_{t} q\right)+\kappa^{2} y\left\|\partial_{t} w\right\|^{2}+ \\
+ & L\left(\partial_{t} u, \partial_{t} q\right)+\kappa L\left(\partial_{t} u, \partial_{t} w\right)+\frac{1}{2}\left(f^{\prime \prime}(w), \partial_{t} w|q|^{2}\right)=: H .
\end{aligned}
$$

Arguing in a similar manner as in the proof of (A.9) we have

$$
C_{v}\left\|\xi_{q}\right\|_{\mathcal{E}}^{2} \leq \Lambda
$$

for some $C_{v}$, as long as $L=L(y, v, f)$ is large enough. Using the growth condition of $f^{\prime \prime}$ (see (H1)), the dissipative estimate for $u$ (1.3), energy estimate for $w$ (Lemma A.1) and arguing as in the proof of (A.10), the right-hand side $H(t)$ can be estimated as follows:

$$
H \leq \frac{M_{L}\left(\|\mathcal{B}\|_{\mathcal{E}}\right)}{\delta}+\delta\left\|\xi_{q}\right\|_{\mathcal{E}}^{2}+\frac{M_{L}\left(\|\mathcal{B}\|_{\mathcal{E}}\right)}{\delta}\left\|\partial_{t} w\right\|^{2}\left\|\xi_{q}\right\|_{\mathcal{E}}^{2},
$$

for any $\delta>0$. Choosing $0<\kappa<\frac{y}{2}, \delta$ small, and collecting (A.13), (A.14), (A.15) we derive

$$
\frac{d}{d t} \Lambda+\kappa \Lambda \leq M_{L}\left(\|\mathcal{B}\|_{\mathcal{E}}\right)+M_{L}\left(\|\mathcal{B}\|_{\mathcal{E}}\right)\left\|\partial_{t} w\right\|^{2} \Lambda .
$$

Consequently, using Lemma A.2 (with small enough $\mu$ ) and applying the modified Gronwall's lemma we determine that

$$
\left\|\nabla \partial_{t} w(t)\right\|+\left\|\partial_{t}^{2} w(t)\right\| \leq M_{L}\left(\|\mathcal{B}\|_{\mathcal{E}}\right), \quad t \geq 0
$$

It now readily follows that

$$
\|\operatorname{div}(a \nabla w)\| \leq M_{L}\left(\|\mathcal{B}\|_{\mathcal{E}}\right), \quad t \geq 0 .
$$

Indeed, by rewriting equation (A.2) in the form

$$
-\operatorname{div}(a \nabla w)=-\partial_{t}^{2} w-y \partial_{t} w-L w-f(w)+L u+g=: H, \quad x \in \Omega, t \geq 0,
$$

then due to Theorem 1.1, Lemma A.1 and (A.16) we see that $\|H(t)\| \leq M_{L}\left(\|\mathcal{B}\|_{\mathcal{E}}\right)$. Hence, the proof is complete.

\section{B Proof of Theorem 6.2}

The proof of Theorem 6.2 is an adaptation of a construction for exponential attractors presented in [29, Theorem 2.10]. The difference here is one needs to keep track on the parameter dependence of all the sets used in the construction and incorporate the fact we compare the symmetric distance in a topology different to that in which the exponential attractors are constructed. For the reader's convenience we shall provide the details here.

\section{B.1 Construction of the exponential attractors.}

Let us introduce notations for the 'starting' $\operatorname{cover} \mathfrak{U}_{\varepsilon}\left(\frac{1}{K}, R\right)$ and the 'model' $\operatorname{cover} \mathfrak{U}_{\varepsilon}\left(\frac{1}{4 K}, 1\right)$ :

$$
\mathcal{V}_{0}(\varepsilon):=\mathcal{U}_{\varepsilon}\left(\frac{1}{K}, R\right), \quad \mathcal{U}(\varepsilon):=\mathcal{U}_{\varepsilon}\left(\frac{1}{4 K}, 1\right)=\left\{\xi_{i \varepsilon}\right\}_{i=1}^{N}, \quad \varepsilon \geq 0,
$$

where $N_{0}:=\operatorname{card} \mathcal{V}_{0}(\varepsilon)=N\left(\frac{1}{K}, R\right)$ and $N:=N\left(\frac{1}{4 K}, 1\right)$ are, by assumption, independent of $\varepsilon \geq 0$. 
We shall begin with constructing a family of sets $\mathcal{V}_{k}(\varepsilon), k \in \mathbb{N}$, that satisfy ${ }^{5}$

$$
\mathcal{V}_{k}(\varepsilon) \subset \mathcal{O}\left(B_{\varepsilon}\right), S_{\varepsilon}(k) B_{\varepsilon} \subset \bigcup_{\xi \in \mathcal{V}_{k}(\varepsilon)} B_{\varepsilon}\left(\xi, \frac{1}{K}\left(\frac{3}{4}\right)^{k}\right), \quad k \in \mathbb{N}, \varepsilon \geq 0 .
$$

Note that, by the assumptions of Theorem 6.2, the above property holds for $k=0$. We now assume that the set $\mathcal{V}_{k}(\varepsilon)$ exists, for some fixed $k$, and are going to construct from it the set $\mathcal{V}_{k+1}(\varepsilon)$. From (B.1) it follows that

$$
S_{\varepsilon}(k+1) B_{\varepsilon} \subset \bigcup_{\xi \in \mathcal{V}_{k}(\varepsilon)} S_{\varepsilon} B_{\varepsilon}\left(\xi, \frac{1}{R}\left(\frac{3}{4}\right)^{k}\right), \varepsilon \geq 0 .
$$

Let us consider an element $S_{\varepsilon} \zeta \in S_{\varepsilon} B_{\varepsilon}\left(\xi, \frac{1}{K}\left(\frac{3}{4}\right)^{k}\right)$ for some $\xi \in \mathcal{V}_{k}(\varepsilon)$. Due to the splitting (6.1) we have

$$
S_{\varepsilon} \zeta-S_{\varepsilon} \xi=v_{\varepsilon}+w_{\varepsilon}, \quad\left\|v_{\varepsilon}\right\|_{\varepsilon} \leq \frac{1}{2 K}\left(\frac{3}{4}\right)^{k}, \quad\left\|w_{\varepsilon}\right\|_{\mathcal{E}_{\varepsilon}^{1}} \leq\left(\frac{3}{4}\right)^{k}, \quad \varepsilon \geq 0 .
$$

Therefore, by using the model cover $U(\varepsilon)$ of $B_{\mathcal{E}_{\varepsilon}^{1}}(0,1)$, we see that

$$
w_{\varepsilon} \in B_{\mathcal{E}_{\varepsilon}^{1}}\left(0,\left(\frac{3}{4}\right)^{k}\right) \subset \bigcup_{i=1}^{N} B_{\varepsilon}\left(\left(\frac{3}{4}\right)^{k} \xi_{i \varepsilon}, \frac{1}{4 K}\left(\frac{3}{4}\right)^{k}\right) .
$$

Since $S_{\varepsilon} \zeta=S_{\varepsilon} \xi+v_{\varepsilon}+w_{\varepsilon}$ we deduce that

$$
S_{\varepsilon}(k+1) B_{\varepsilon} \subset \bigcup_{\xi \in \mathcal{V}_{k}(\varepsilon)} \bigcup_{i=1}^{N} B_{\varepsilon}\left(S_{\varepsilon} \xi+\left(\frac{3}{4}\right)^{k} \xi_{i \varepsilon}, \frac{1}{K}\left(\frac{3}{4}\right)^{k+1}\right), \quad \varepsilon \geq 0 .
$$

As $\left\|\zeta_{i, \varepsilon}\right\|_{\mathcal{E}_{\varepsilon}^{1}} \leq \delta_{1}$ we conclude that (B.1) holds for

$$
\mathcal{V}_{k+1}(\varepsilon):=S_{\varepsilon} \mathcal{V}_{k}(\varepsilon)+\left(\frac{3}{4}\right)^{k} U(\varepsilon) \subset \mathcal{O}\left(B_{\varepsilon}\right), \quad k \in \mathbb{Z}_{+}, \varepsilon \geq 0 .
$$

Now, it is straightforward to verify the following properties of $\mathcal{V}_{k}(\varepsilon)$ :

$$
\left\{\begin{array}{l}
\operatorname{card} \mathcal{V}_{k}(\varepsilon)=N_{0} N^{k}, \\
\operatorname{dist}_{\mathcal{E}}\left(S_{\varepsilon}(k) B_{\varepsilon}, V_{k}(\varepsilon)\right) \leq \frac{1}{R}\left(\frac{3}{4}\right)^{k}, \\
\operatorname{dist}_{\mathcal{E}}^{S}\left(\mathcal{V}_{k+1}(\varepsilon), S_{\varepsilon} \mathcal{V}_{k}(\varepsilon)\right) \leq c_{0} \delta_{1}\left(\frac{3}{4}\right)^{k},
\end{array} \quad k \in \mathbb{N}, \varepsilon \geq 0 .\right.
$$

Based on the sets $\mathcal{V}_{k}(\varepsilon)$ we construct the sets $E_{k}(\varepsilon) \subset \mathcal{O}\left(B_{\varepsilon}\right)$ :

$$
E_{1}(\varepsilon):=\mathcal{V}_{1}(\varepsilon), \quad E_{k+1}(\varepsilon):=\mathcal{V}_{k+1}(\varepsilon) \cup S_{\varepsilon} E_{k}(\varepsilon), \quad k \in \mathbb{N}, \varepsilon \geq 0,
$$

that clearly satisfy

$$
\left\{\begin{array}{l}
\operatorname{card} E_{k}(\varepsilon) \leq k N_{0} N^{k}, \\
S_{\varepsilon} E_{k}(\varepsilon) \subset E_{k+1}(\varepsilon), \\
\operatorname{dist}_{\mathcal{E}}\left(S_{\varepsilon}(k) B_{\varepsilon}, E_{k}(\varepsilon)\right)<\frac{1}{R}\left(\frac{3}{4}\right)^{k},
\end{array} \quad k \in \mathbb{N}, \varepsilon \geq 0 .\right.
$$

We shall now demonstrate that the sets

$$
\mathcal{M}^{\varepsilon}:=\left[\hat{\mathcal{M}}^{\varepsilon}\right]_{\varepsilon}, \quad \hat{\mathcal{M}}^{\varepsilon}:=\bigcup_{k=1}^{\infty} E_{k}(\varepsilon), \varepsilon \geq 0
$$

are exponential attractors for the discrete dynamical systems $\left(B_{\varepsilon}, S_{\varepsilon}\right)$. To this end we use the following result.

$\overline{5 \text { Here } S_{\varepsilon}}(k)$ denotes the $k^{\text {th }}$ iteration of $S_{\varepsilon}$. 
Lemma B.1. Let the assumptions of Theorem 6.2 hold and the sets $E_{k}(\varepsilon), k \in \mathbb{N}, \varepsilon \geq 0$, be given by (B.4). Then, there exist constants $M_{1}=M_{1}\left(c_{0}, K, \delta_{1}\right)>0$ and $\omega=\omega\left(c_{0}, K, \delta_{1}\right) \in(0,1)$ (both independent of $\varepsilon$ ) such that for all $\varepsilon \geq 0$ we have

$$
\operatorname{dist}_{\varepsilon}\left(E_{k}(\varepsilon), S_{\varepsilon}(n) B_{\varepsilon}\right) \leq M_{1}\left(\frac{3}{4}\right)^{\omega k}, \quad \text { for all } n \in \mathbb{N}, k \in \mathbb{N}: k \geq \frac{n}{\omega} .
$$

The proof of this lemma, basically, repeats the proof of Lemma 2.3 from [29], so we omit the proof.

Now, we are ready to verify that the constructed sets $\mathcal{M}^{\varepsilon}$ satisfy Definition 6.1. The positive invariance and the uniform exponential attraction property (with $\sigma=\ln \left(\frac{4}{3}\right)$ )

$$
\operatorname{dist}_{\mathcal{E}}\left(S_{\varepsilon}(k) B_{\varepsilon}, \mathcal{M}^{\varepsilon}\right) \leq \frac{1}{K}\left(\frac{3}{4}\right)^{k}, \quad k \in \mathbb{N}, \varepsilon \geq 0,
$$

follow directly from (B.5) $)_{2}$, (B.5) ${ }_{3}$ and (B.6). From the construction it also follows that $\mathcal{M}^{\varepsilon} \subset \mathcal{O}\left(B_{\varepsilon}\right)$ and thus $\mathcal{M}^{\varepsilon}$ is compact in $\mathcal{E}$ for every $\varepsilon \geq 0$. Let us check that $\operatorname{dim}_{f}\left(\mathcal{M}^{\varepsilon}, \mathcal{E}\right) \leq D$ uniformly with respect to $\varepsilon \geq 0$. To this end we need to estimate the minimal number $N_{r}\left(\mathcal{M}^{\varepsilon}, \mathcal{E}\right)$ of open balls with radius $r>0$ in $\varepsilon$ needed to cover $\mathcal{M}^{\varepsilon}$. Note that, since the cover is open, $N_{r}\left(\mathcal{M}^{\varepsilon}, \varepsilon\right)=N_{r}\left(\hat{\mathcal{M}}^{\varepsilon}, \varepsilon\right)$. We argue that for any $r>0$ there exist $k_{r} \in \mathbb{N}$ and $n_{r} \in \mathbb{N}$ (independent of $\varepsilon$ ) such that

$$
\operatorname{dist}_{\mathcal{E}}\left(\bigcup_{k=k_{r}+1}^{\infty} E_{k}(\varepsilon), \nu_{n_{r}}(\varepsilon)\right)<r, \quad \varepsilon \geq 0
$$

Indeed, let $k_{r}$ and $n_{r}$ be parameters, then by the triangle inequality we have

$$
\operatorname{dist}_{\mathcal{E}}\left(\bigcup_{k=k_{r}+1}^{\infty} E_{k}(\varepsilon), v_{n_{r}}(\varepsilon)\right) \leq \operatorname{dist}_{\varepsilon}\left(\bigcup_{k=k_{r}+1}^{\infty} E_{k}(\varepsilon), S_{\varepsilon}\left(n_{r}\right) B_{\varepsilon}\right)+\operatorname{dist}_{\varepsilon}\left(S_{\varepsilon}\left(n_{r}\right) B_{\varepsilon}, V_{n_{r}}(\varepsilon)\right), \varepsilon \geq 0 .
$$

Using (B.3) $)_{2}$ and taking $n_{r} \geq{ }^{6}\left\lfloor\frac{1}{\ln (4 / 3)} \ln \left(\frac{2}{r K}\right)\right\rfloor \vee 0+1$ we obtain

$$
\operatorname{dist}_{\mathcal{E}}\left(S_{\varepsilon}\left(n_{r}\right) B_{\varepsilon}, \mathcal{V}_{n_{r}}(\varepsilon)\right)<\frac{r}{2}, \quad \varepsilon \geq 0 .
$$

Also applying Lemma B.1 for any $k_{r} \in \mathbb{N}$ such that $k_{r} \geq \frac{n_{r}}{\omega}$, we find that

$$
\operatorname{dist}_{\varepsilon}\left(\bigcup_{k=k_{r}+1}^{\infty} E_{k}(\varepsilon), S_{\varepsilon}\left(n_{r}\right) B_{\varepsilon}\right) \leq M_{1}\left(\frac{3}{4}\right)^{\omega k_{r}} \leq M_{1}\left(\frac{3}{4}\right)^{n_{r}}<\frac{r}{2}, \quad \varepsilon \geq 0,
$$

if $n_{r} \geq\left\lfloor\frac{1}{\ln (4 / 3)} \ln \left(\frac{2 M_{1}}{r}\right)\right\rfloor \vee 0+1$. Therefore (B.8) is valid for $n_{r}$ and $k_{r}$ of the form

$$
n_{r}=\left\lfloor\frac{1}{\ln (4 / 3)} \ln \left(\frac{1}{r}\right)\right\rfloor \vee 0+C_{1}\left(c_{0}, K, \delta_{1}\right), \quad k_{r}=\left\lfloor\frac{1}{\omega \ln (4 / 3)} \ln \left(\frac{1}{r}\right)\right\rfloor \vee 0+C_{2}\left(c_{0}, K, \omega, \delta_{1}\right) .
$$

Using the control on the number of elements for $\mathcal{V}_{k}(\varepsilon)$ and $E_{k}(\varepsilon)$, (B.6) and (B.8) we can estimate $N_{r}\left(\hat{\mathcal{M}}^{\varepsilon}, \mathcal{\varepsilon}\right)$ as follows

$$
N_{r}\left(\hat{\mathcal{M}}^{\varepsilon}, \varepsilon\right) \leq \sum_{k=1}^{k_{r}} \operatorname{card} E_{k}(\varepsilon)+\operatorname{card} V_{n_{r}}(\varepsilon) \leq \sum_{k=1}^{k_{r}} k N_{0} N^{k}+N_{0} N^{n_{r}} \leq\left(k_{r}^{2}+1\right) N_{0} N^{k_{r}} .
$$

This estimate readily yields

$$
\operatorname{dim}_{f}\left(\mathcal{M}^{\varepsilon}, \mathcal{E}\right):=\limsup _{r \rightarrow+0} \frac{\ln N_{r}\left(\mathcal{M}^{\varepsilon}, \mathcal{E}\right)}{\ln \left(\frac{1}{r}\right)} \leq \frac{\ln N}{\omega \ln (4 / 3)}=: D, \quad \varepsilon \geq 0 .
$$

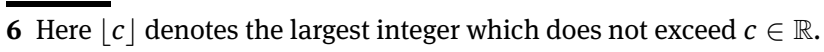




\section{B.2 Estimate on the symmetric distance}

Derivation of the estimate on the symmetric distance $\operatorname{dist}_{\mathcal{E}^{-1}}^{s}\left(\mathcal{M}^{\varepsilon}, \mathcal{M}^{0}\right)$ relies on the following result.

Lemma B.2. Let the assumptions of Theorem 6.2 hold and the sets $E_{k}(\varepsilon), k \in \mathbb{N}, \varepsilon \geq 0$, be given by (B.4). Then for all $k \in \mathbb{N}$ and $\varepsilon \geq 0$ the following estimate

$$
\begin{array}{r}
\operatorname{dist}_{\mathcal{E}^{-1}}^{s}\left(E_{k}(\varepsilon), E_{k}(0)\right) \leq M L^{k}\left(\sup _{\xi \in \mathcal{O}\left(B_{\varepsilon}\right)}\left\|S_{\varepsilon} \xi-S_{0} \xi\right\|_{\mathcal{E}^{-1}}+\operatorname{dist}_{\mathcal{E}^{-1}}^{s}\left(\mathcal{U}_{\varepsilon}\left(\frac{1}{4 K}, 1\right), \mathcal{U}_{0}\left(\frac{1}{4 K}, 1\right)\right)\right. \\
+\operatorname{dist}_{\mathcal{E}^{-1}}^{s}\left(\mathcal{U}_{\varepsilon}\left(\frac{1}{K}, R\right), \mathcal{U}_{0}\left(\frac{1}{K}, R\right)\right),
\end{array}
$$

holds for some constant $M=M(L)$ independent of $\varepsilon$ and $k$.

Proof. Fix $\varepsilon \geq 0$.

Step 1. We first establish (B.10) for the sets $\mathcal{V}_{k}(\varepsilon), \mathcal{V}_{k}(0)$. To this end it is convenient to introduce the notations

$$
\begin{aligned}
& d_{k}:=\operatorname{dist}_{\mathcal{E}^{-1}}^{S}\left(\mathcal{V}_{k}(\varepsilon), \mathcal{V}_{k}(0)\right), k \in \mathbb{Z}_{+}, \quad \hat{d}_{0}:=\operatorname{dist}_{\mathcal{E}^{-1}}^{s}(\mathcal{U}(\varepsilon), \mathcal{U}(0)) ; \\
& s_{0}:=\sup _{\xi \in \mathcal{O}\left(B_{\varepsilon}\right)}\left\|S_{\varepsilon} \xi-S_{0} \xi\right\|_{\mathcal{E}^{-1}} .
\end{aligned}
$$

It is sufficient to establish that the following recurrent chain of inequalities

$$
d_{k+1} \leq s_{0}+\hat{d}_{0}+L d_{k}, \quad k \in \mathbb{Z}_{+} .
$$

Indeed, upon iterating these inequalities one finds

$$
d_{k} \leq \frac{L^{k+1}-1}{L-1}\left(s_{0}+\hat{d}_{0}+d_{0}\right), \quad k \in \mathbb{Z}_{+} .
$$

Let us prove (B.11). Note that, from the construction of $\mathcal{V}_{k}(\varepsilon)$ (B.2), we readily have the following inequalities

$$
\operatorname{dist}_{\mathcal{E}^{-1}}^{s}\left(\mathcal{V}_{k+1}(\varepsilon), \mathcal{V}_{k+1}(0)\right) \leq \operatorname{dist}_{\mathcal{E}^{-1}}^{S}\left(S_{\varepsilon} \mathcal{V}_{k}(\varepsilon), S_{0} \mathcal{V}_{k}(0)\right)+\hat{d}_{0}, \quad k \in \mathbb{Z}_{+} .
$$

Let us now verify the inequality

$$
\operatorname{dist}_{\mathcal{E}^{-1}}^{s}\left(S_{\varepsilon} A, S_{0} C\right) \leq S_{0}+L \operatorname{dist}_{\mathcal{E}^{-1}}^{s}(A, C), \quad \text { for all } A \subset \mathcal{O}\left(B_{\varepsilon}\right), C \subset \mathcal{O}\left(B_{0}\right) .
$$

Fixing arbitrary $a \in A, c \in C$ and using Lipschitz continuity of $S_{0}$ in $\mathcal{E}^{-1}$ we obtain

$$
\left\|S_{\varepsilon} a-S_{0} c\right\|_{\mathcal{E}^{-1}} \leq\left\|S_{\varepsilon} a-S_{0} a\right\|_{\mathcal{E}^{-1}}+\left\|S_{0} a-S_{0} c\right\|_{\mathcal{E}^{-1}}
$$

$$
\leq\left\|S_{\varepsilon} a-S_{0} a\right\|_{\mathcal{E}^{-1}}+L\|a-c\|_{\mathcal{E}^{-1}} \leq S_{0}+L\|a-c\|_{\mathcal{E}^{-1}} .
$$

Consequently (B.14) holds. Hence, upon combining (B.13) with (B.14), we deduce (B.11) and step 1 is complete.

Step 2. We claim that the sets $E_{k}(\varepsilon), E_{k}(0)$ satisfy the same inequality as in (B.12), namely

$$
\operatorname{dist}_{\mathcal{E}^{-1}}^{s}\left(E_{k}(\varepsilon), E_{k}(0)\right) \leq \frac{L^{k+1}-1}{L-1}\left(s_{0}+d_{0}+\hat{d}_{0}\right), k \in \mathbb{N} .
$$

Since $E_{1}(\varepsilon)=V_{1}(\varepsilon)$ for all $\varepsilon \geq 0$, the above inequality is true for $k=1$. Assume (B.15) holds for $k=m$ and let us verify it for $k=m+1$. It is straightforward to check that for any $A_{1}, A_{2} \subset \mathcal{O}\left(B_{\varepsilon}\right), C_{1}, C_{2} \subset \mathcal{O}\left(B_{0}\right)$ the following inequality

$$
\operatorname{dist}_{\mathcal{E}^{-1}}^{S}\left(A_{1} \cup A_{2}, C_{1} \cup C_{2}\right) \leq \operatorname{dist}_{\mathcal{E}^{-1}}^{S}\left(A_{1}, C_{1}\right) \vee \operatorname{dist}_{\mathcal{E}^{-1}}^{s}\left(A_{2}, C_{2}\right)
$$

holds. Therefore, due to (B.4), it is enough to show that

$$
\operatorname{dist}_{\mathcal{E}^{-1}}^{S}\left(S_{\varepsilon} E_{m}(\varepsilon), S_{0} E_{m}(0)\right) \leq\left(s_{0}+d_{0}+\hat{d}_{0}\right) \frac{L^{m+2}-1}{L-1} .
$$


This inequality is a direct consequence of (B.14) and the induction assumption. Indeed, we compute

$$
\begin{aligned}
\operatorname{dist}_{\mathcal{E}^{-1}}^{S}\left(S_{\varepsilon} E_{m}(\varepsilon), S_{0} E_{m}(0)\right) & \leq s_{0}+L \operatorname{dist}_{\mathcal{E}^{-1}}^{S}\left(E_{m}(\varepsilon), E_{m}(0)\right) \\
& \leq\left(s_{0}+d_{0}+\hat{d}_{0}\right)\left(1+L \frac{L^{m+1}-1}{L-1}\right)=\left(s_{0}+d_{0}+\hat{d}_{0}\right) \frac{L^{m+2}-1}{L-1},
\end{aligned}
$$

as required. Hence, inequality (B.15) yields the desired result with $M(L)=\frac{L}{L-1}$.

We proceed to the proof of the estimate (6.2) on the distance dist $\mathrm{E}^{-1}\left(\mathcal{M}^{\varepsilon}, \mathcal{M}^{0}\right)$. We fix $\varepsilon \geq 0$ and set

$$
\tilde{d}:=\sup _{\xi \in \mathcal{O}\left(B_{\varepsilon}\right)}\left\|S_{\varepsilon} \xi-S_{0} \xi\right\|_{\mathcal{E}^{-1}}+\operatorname{dist}_{\mathcal{E}^{-1}}^{s}\left(B_{\varepsilon}, B_{0}\right)+\operatorname{dist}_{\mathcal{E}^{-1}}^{s}\left(\mathcal{U}_{\mathcal{E}}\left(\frac{1}{4 K}, 1\right), \mathcal{U}_{0}\left(\frac{1}{4 K}, 1\right)\right)+\operatorname{dist}_{\mathcal{E}^{-1}}^{s}\left(\mathcal{U}_{\varepsilon}\left(\frac{1}{K}, R\right), \mathcal{U}_{0}\left(\frac{1}{K}, R\right)\right) .
$$

In fact, we will only demonstrate how to obtain the estimate (6.2) for $\operatorname{dist}_{\varepsilon^{-1}}\left(\mathcal{M}^{\varepsilon}, \mathcal{M}^{0}\right)$ as the other side (dist ${ }_{\mathcal{E}^{-1}}\left(\mathcal{M}^{0}, \mathcal{M}^{\varepsilon}\right)$ ) can be done similarly. Let $k \in \mathbb{N}$ be arbitrary and fix $\xi_{\varepsilon} \in E_{k}(\varepsilon)$. Due to the just proved Lemma B.2 we have

$$
\operatorname{dist}_{\mathcal{E}^{-1}}\left(\xi_{\varepsilon}, \mathcal{M}^{0}\right) \leq \operatorname{dist}_{\mathcal{E}^{-1}}\left(\xi_{\varepsilon}, E_{k}(0)\right) \leq M L^{k} \tilde{d}, \quad k \in \mathbb{N}, \varepsilon \geq 0 .
$$

On the other hand, we will show below that

$$
\operatorname{dist}_{\mathcal{E}^{-1}}\left(\xi_{\varepsilon}, \mathcal{M}^{0}\right) \leq M\left(\tilde{d} L^{\frac{n}{\omega}}+\left(\frac{3}{4}\right)^{n}\right), \quad \text { for all } n \in \mathbb{N}, k \in \mathbb{N}: k \geq \frac{n}{\omega} .
$$

Using (B.18) for $k \leq \frac{n}{\omega}$ and (B.19) we deduce that

$$
\operatorname{dist}_{\mathcal{E}^{-1}}\left(\xi_{\varepsilon}, \mathcal{M}^{0}\right) \leq M\left(\tilde{d} L^{\frac{n}{\omega}}+\left(\frac{3}{4}\right)^{n}\right),
$$

for some $M=M\left(c_{0}, c_{-1}, K, L, \delta_{1}\right)$ which is independent of $\varepsilon$. Optimizing $n$ in the above inequality, for example taking $n=\left\lfloor\frac{\omega}{\omega \ln (4 / 3)+L} \ln \left(\frac{\omega \ln (4 / 3)}{\tilde{d} \ln L}\right)\right\rfloor \vee 0$, we conclude the desired estimate (6.2) with $\varkappa=\frac{\omega \ln (4 / 3)}{\omega \ln (4 / 3)+\ln (L)}$.

It remains to prove (B.19). By the triangle inequality we have

$$
\operatorname{dist}_{\mathcal{E}^{-1}}\left(\xi_{\varepsilon}, \mathcal{M}^{0}\right) \leq \operatorname{dist}_{\mathcal{E}^{-1}}\left(\xi_{\varepsilon}, S_{\varepsilon}(n) B_{\varepsilon}\right)+\operatorname{dist}_{\mathcal{E}^{-1}}\left(S_{\varepsilon}(n) B_{\varepsilon}, S_{0}(n) B_{0}\right)+\operatorname{dist}_{\mathcal{E}^{-1}}\left(S_{0}(n) B_{0}, \mathcal{M}^{0}\right) .
$$

Let us estimate each of the terms on the right hand side of (B.21) separately. Using Lemma B.1 and considering $k \geq \frac{n}{\omega}$ we obtain

$$
\operatorname{dist}_{\mathcal{E}^{-1}}\left(\xi_{\varepsilon}, S_{\varepsilon}(n) B_{\varepsilon}\right) \leq M_{1}\left(\frac{3}{4}\right)^{n}, \quad \text { for all } n \in \mathbb{N}, k \in \mathbb{N}: k \geq \frac{n}{\omega} .
$$

Iterating (B.14) we find

$$
\operatorname{dist}_{\mathcal{E}^{-1}}\left(S_{\varepsilon}(n) B_{\varepsilon}, S_{0}(n) B_{0}\right) \leq\left(s_{0}+\operatorname{dist}_{\mathcal{E}^{-1}}^{S}\left(B_{\varepsilon}, B_{0}\right)\right) \frac{L^{n+1}}{L-1} \leq \tilde{d} \frac{L^{n+1}}{L-1} .
$$

Finally, due to the continuous embedding $\mathcal{E} \subset \mathcal{E}^{-1}$ (assumption (2)) and the exponential attraction property of $\mathcal{M}^{0}$ (B.7) we see that

$$
\operatorname{dist}_{\mathcal{E}^{-1}}\left(S_{0}(n) B_{0}, \mathcal{M}^{0}\right) \leq c_{-1} \operatorname{dist}_{\mathcal{E}}\left(S_{0}(n) B_{0}, \mathcal{M}^{0}\right) \leq c_{-1} \frac{1}{K}\left(\frac{3}{4}\right)^{n}, n \in \mathbb{N} .
$$

Hence (B.19) holds and the proof is complete.

\section{Proof of Theorem 6.4}

Derivation of the estimate on the symmetric distance with correction relies on the following interesting modification of Lemma B.2. 
Lemma C.1. Let the assumptions of Theorem 6.4 hold and the sets $E_{k}(\varepsilon), k \in \mathbb{N}, \varepsilon \geq 0$, be given by (B.4). Then for all $k \in \mathbb{N}$ and $\varepsilon \geq 0$ the following estimate

$$
\begin{aligned}
\operatorname{dist}_{\mathcal{E}}^{s}\left(E_{k}(\varepsilon), T_{\varepsilon} E_{k}(0)\right) \leq M L^{k}\left(\sup _{\xi \in \mathcal{O}\left(B_{0}\right)}\left\|S_{\varepsilon} \Pi_{\varepsilon}^{-1} \xi-T_{\varepsilon} S_{0} \xi\right\|_{\mathcal{E}}+\sup _{\xi \in \mathcal{O}\left(B_{0}\right)}\left\|T_{\varepsilon} \xi-\Pi_{\varepsilon}^{-1} \xi\right\|_{\mathcal{E}}\right. \\
\\
\left.\quad+\operatorname{dist}_{\mathcal{E}}^{s}\left(\mathcal{U}_{\varepsilon}\left(\frac{1}{4 R}, 1\right), T_{\varepsilon} \mathcal{U}_{0}\left(\frac{1}{4 R}, 1\right)\right)+\operatorname{dist}_{\mathcal{E}}^{s}\left(\mathcal{U}_{\varepsilon}\left(\frac{1}{R}, R\right), T_{\varepsilon} \mathcal{U}_{0}\left(\frac{1}{R}, R\right)\right)\right),
\end{aligned}
$$

holds with some constant $M=M(L)$ which is independent of $\varepsilon$ and $k$.

Proof. We follow the strategy of Lemma B.2 and fix $\varepsilon \geq 0$.

We first derive an estimate on the distance between $\mathcal{V}_{k}(\varepsilon)$ and $\mathrm{T}_{\varepsilon} \mathcal{V}_{k}(0)$. Let us introduce the notations

$$
\begin{array}{r}
d_{k}:=\operatorname{dist}_{\mathcal{E}}^{s}\left(\mathcal{V}_{k}(\varepsilon), \mathrm{T}_{\mathcal{\varepsilon}} \mathcal{V}_{k}(0)\right), k \in \mathbb{Z}_{+}, \quad \hat{d}_{0}:=\operatorname{dist}_{\mathcal{E}}^{s}\left(\mathcal{U}(\varepsilon), \mathrm{T}_{\varepsilon} U(0)\right) ; \\
s_{0}:=\sup _{\xi \in \mathcal{O}\left(B_{0}\right)}\left\|S_{\varepsilon} \Pi_{\varepsilon}^{-1} \xi-\mathrm{T}_{\varepsilon} S_{0} \xi\right\|_{\mathcal{E}}+L \sup _{\xi \in \mathcal{O}\left(B_{0}\right)}\left\|\Pi_{\varepsilon}^{-1} \xi-\mathrm{T}_{\varepsilon} \xi\right\|_{\mathcal{E}} .
\end{array}
$$

We are going to verify the recurrent chain of inequalities

$$
d_{k+1} \leq s_{0}+\hat{d}_{0}+L d_{k}, \quad k \in \mathbb{Z}_{+} .
$$

From the construction of $\mathcal{V}_{k}(\varepsilon)($ B.2) we see

$$
\operatorname{dist}_{\varepsilon}^{S}\left(\mathcal{V}_{k+1}(\varepsilon), \mathrm{T}_{\varepsilon} \mathcal{V}_{k+1}(0)\right) \leq \operatorname{dist}_{\varepsilon}^{S}\left(S_{\varepsilon} \mathcal{V}_{k}(\varepsilon), \mathrm{T}_{\varepsilon} S_{0} \mathcal{V}_{k}(0)\right)+\hat{d}_{0}, k \in \mathbb{Z}_{+} .
$$

We now argue that

$$
\operatorname{dist}_{\mathcal{E}}^{s}\left(S_{\varepsilon} A, \mathrm{~T}_{\varepsilon} S_{0} C\right) \leq S_{0}+L \operatorname{dist}_{\mathcal{E}}^{s}\left(A, \mathrm{~T}_{\varepsilon} C\right), \quad \text { for all } A \subset \mathcal{O}\left(B_{\varepsilon}\right), C \subset \mathcal{O}\left(B_{0}\right) .
$$

Indeed, fixing $a \in A, c \in C$ and using the uniform (with respect to $\varepsilon>0$ ) Lipschitz continuity of $S_{\varepsilon}$ in $\mathcal{E}$ (assumption (5) of Theorem 6.4) we compute

$$
\begin{aligned}
\left\|S_{\varepsilon} a-\mathrm{T}_{\varepsilon} S_{0} c\right\|_{\varepsilon} & \leq\left\|S_{\varepsilon} a-S_{\varepsilon} \Pi_{\varepsilon}^{-1} c\right\|_{\mathcal{E}}+\left\|S_{\varepsilon} \Pi_{\varepsilon}^{-1} c-\mathrm{T}_{\varepsilon} S_{0} c\right\|_{\varepsilon} \\
& \leq L\left\|a-\Pi_{\varepsilon}^{-1} c\right\|_{\mathcal{E}}+\left\|S_{\varepsilon} \Pi_{\varepsilon}^{-1} c-\mathrm{T}_{\varepsilon} S_{0} c\right\|_{\varepsilon} \\
& \leq L\left\|a-\mathrm{T}_{\varepsilon} c\right\|_{\mathcal{E}}+L\left\|\mathrm{~T}_{\varepsilon} c-\Pi_{\varepsilon}^{-1} c\right\|_{\mathcal{E}}+\left\|S_{\varepsilon} \Pi_{\varepsilon}^{-1} c-\mathrm{T}_{\varepsilon} S_{0} c\right\|_{\varepsilon} .
\end{aligned}
$$

The above inequality, obviously, implies (C.4). Combining (C.3) and (C.4) we establish the recurrent inequalities (C.2) which yield

$$
d_{k} \leq\left(s_{0}+d_{0}+\hat{d}_{0}\right) \frac{L^{k+1}-1}{L-1}, \quad k \in \mathbb{Z}_{+} .
$$

To derive the estimate (C.1) on the distance $\operatorname{dist}_{\mathcal{E}}^{S}\left(E_{k}(\varepsilon), \mathrm{T}_{\varepsilon} E_{k}(0)\right)$ we simply argue as in Step 2 of Lemma B.2.

We are ready to prove the theorem. We fix $\varepsilon \geq 0$ and set

$$
\begin{aligned}
\tilde{d}:=\sup _{\xi \in \mathcal{O}\left(B_{0}\right)}\left\|S_{\varepsilon} \Pi_{\varepsilon}^{-1} \xi-\mathrm{T}_{\varepsilon} S_{0} \xi\right\|_{\mathcal{E}} & +\sup _{\xi \in \mathcal{O}\left(B_{0}\right)}\left\|\mathrm{T}_{\varepsilon} \xi-\Pi_{\varepsilon}^{-1} \xi\right\|_{\mathcal{E}} \\
& \quad+\operatorname{dist}_{\mathcal{E}}^{s}\left(\mathcal{U}_{\varepsilon}\left(\frac{1}{4 R}, 1\right), \mathrm{T}_{\varepsilon} \mathcal{U}_{0}\left(\frac{1}{4 R}, 1\right)\right)+\operatorname{dist}_{\mathcal{E}}^{s}\left(\mathcal{U}_{\varepsilon}\left(\frac{1}{R}, R\right), \mathrm{T}_{\varepsilon} \mathcal{U}_{0}\left(\frac{1}{R}, R\right)\right) .
\end{aligned}
$$

As in the proof of Theorem 6.2 we will only consider $\operatorname{dist}_{\mathcal{E}}\left(\mathcal{M}^{\varepsilon}, \mathrm{T}_{\varepsilon} \mathcal{M}^{0}\right)$ as the other side can argued in a similar manner. Let $k \in \mathbb{N}$ and $\xi_{\varepsilon} \in E_{k}(\varepsilon)$ be fixed. Then according to Lemma C.1 we have

$$
\operatorname{dist}_{\mathcal{E}}\left(\xi_{\varepsilon}, \mathrm{T}_{\varepsilon} \mathcal{M}^{0}\right) \leq \operatorname{dist}_{\varepsilon}\left(\xi_{\varepsilon}, \mathrm{T}_{\varepsilon} E_{k}(0)\right) \leq M L^{k} \tilde{d}, \quad k \in \mathbb{N}, \varepsilon \geq 0 .
$$

On the other hand we deduce below that

$$
\operatorname{dist}_{\varepsilon}\left(\xi_{\varepsilon}, \mathrm{T}_{\varepsilon} \mathcal{M}^{0}\right) \leq M\left(\tilde{d} L^{\frac{n}{\omega}}+\left(\frac{3}{4}\right)^{n}\right)+m(\varepsilon), \quad k \geq \frac{n}{\omega}, n \in \mathbb{N},
$$


for $\omega$ given in Lemma B.1. The estimate (C.5) for $k \leq \frac{n}{\omega}$ together with (C.6) implies

$$
\operatorname{dist}_{\varepsilon}\left(\xi_{\varepsilon}, \mathrm{T}_{\varepsilon} \mathcal{M}^{0}\right) \leq M\left((\tilde{d}+m(\varepsilon)) L^{\frac{n}{\omega}}+\left(\frac{3}{4}\right)^{n}\right),
$$

for some $M=M\left(c_{0}, K, L, L_{\mathrm{cor}}, \delta_{1}\right)$ which is independent of $\varepsilon$. Optimizing $n$ in the above inequality provides the desired result.

It remains to prove (C.6). By the triangle inequality we deduce that

$$
\begin{aligned}
& \operatorname{dist}_{\varepsilon}\left(\xi_{\varepsilon}, \mathrm{T}_{\varepsilon} \mathcal{M}^{0}\right) \leq \\
& \quad \operatorname{dist}_{\mathcal{E}}\left(\xi_{\varepsilon}, S_{\varepsilon}(n) B_{\varepsilon}\right)+\operatorname{dist}_{\mathcal{E}}\left(S_{\varepsilon}(n) B_{\varepsilon}, \mathrm{T}_{\varepsilon} S_{0}(n) \Pi_{\varepsilon} B_{\varepsilon}\right)+\operatorname{dist}_{\varepsilon}\left(\mathrm{T}_{\varepsilon} S_{0}(n) \Pi_{\varepsilon} B_{\varepsilon}, \mathrm{T}_{\varepsilon} \mathcal{M}^{0}\right) .
\end{aligned}
$$

The first term on the right hand side of (C.8) can be controlled by Lemma B.1 for $k \geq \frac{n}{\omega}$ :

$$
\operatorname{dist}_{\mathcal{E}}\left(\xi_{\varepsilon}, S_{\varepsilon}(n) B_{\varepsilon}\right) \leq M_{1}\left(\frac{3}{4}\right)^{n} .
$$

By the identity $\Pi_{\varepsilon} B_{\varepsilon}=B_{0}$ (assumption (3) of Theorem 6.4) and iterations of (C.4) we estimate the second term on the right hand side of (C.8):

$$
\operatorname{dist}_{\mathcal{E}}\left(S_{\varepsilon}(n) B_{\varepsilon}, \mathrm{T}_{\varepsilon} S_{0}(n) \Pi_{\varepsilon} B_{\varepsilon}\right)=\operatorname{dist}_{\mathcal{E}}\left(S_{\varepsilon}(n) B_{\varepsilon}, \mathrm{T}_{\varepsilon} S_{0}(n) B_{0}\right) \leq s_{0} \frac{L^{n}-1}{L-1} \leq \tilde{d}_{L-1}^{L} L^{n} .
$$

The last term on the right hand side of (C.8) can be estimated using $\Pi_{\varepsilon} B_{\varepsilon}=B_{0}$ and the property of $\mathrm{T}_{\varepsilon}$ (assumption (4) of Theorem 6.4) and the exponential attraction property of $\mathcal{M}^{0}$ :

$$
\begin{aligned}
\operatorname{dist}_{\varepsilon}\left(\mathrm{T}_{\varepsilon} S_{0}(n) \Pi_{\varepsilon} B_{\varepsilon}, \mathrm{T}_{\varepsilon} \mathcal{M}^{0}\right) & =\operatorname{dist}_{\mathcal{E}}\left(\mathrm{T}_{\varepsilon} S_{0}(n) B_{0}, \mathrm{~T}_{\varepsilon} \mathcal{M}^{0}\right) \\
& \leq L_{\text {cordist }} \operatorname{dis}_{\mathcal{L}}\left(S_{0}(n) B_{0}, \mathcal{M}^{0}\right)+m(\varepsilon) \leq L_{\operatorname{cor}} \frac{1}{K}\left(\frac{3}{4}\right)^{n}+m(\varepsilon) .
\end{aligned}
$$

Hence (C.6) follows from (C.8)-(C.11) and the theorem is proved.

\section{On the refinement of inequality (5.8)}

Let us begin by noting that in Section 5 we were actually in the position to prove the following improvement of inequality (5.8) (in Theorem 5.2).

Proposition D.1. For every $\xi \in B_{\mathcal{E}_{\varepsilon}^{2}}(0, R)$ the inequality

$$
\left\|\partial_{t} S_{\varepsilon}(t) \xi-\partial_{t} S_{0}(t) \Pi_{\varepsilon} \xi\right\|_{\mathcal{E}^{-1}} \leq M e^{K t}\left\|A_{\varepsilon}^{-1}-A_{0}^{-1}\right\|_{\mathcal{L}\left(L^{2}(\Omega)\right)}^{2 / 3}, \quad t \geq 0,
$$

holds for some non-decreasing functions $M=M(R,\|g\|)$ and $K=K(R,\|g\|)$ which are independent of $\varepsilon>0$.

Proof. The proof of this result follows along the same lines as in the proof of Theorem 5.2 except for the following minor alterations:

1. In the uniform bounds (5.9) (due to Theorem 2.5) we actually have

$$
\left\|\partial_{t}^{2} u^{\varepsilon}\right\|_{H_{0}^{1}(\Omega)}^{2}+\left\|\partial_{t}^{2} u^{0}\right\|_{H_{0}^{1}(\Omega)}^{2} \leq M .
$$

2. From (1) we can see that $q^{\varepsilon}=\partial_{t} u^{\varepsilon}-\partial_{t} u^{0}$ satisfies the bound

$$
\left\|\partial_{t} q^{\varepsilon}\right\| \leq\left\|\partial_{t} q^{\varepsilon}\right\|_{H^{-1}(\Omega)}^{1 / 2}\left\|\partial_{t} q^{\varepsilon}\right\|_{H_{0}^{1}(\Omega)}^{1 / 2} \leq M\left\|\partial_{t} q^{\varepsilon}\right\|_{H^{-1}(\Omega)}^{1 / 2},
$$

and so we can improve (5.13) as follows:

$$
\begin{aligned}
\left|\left(A_{0} \partial_{t} u^{\varepsilon}-A_{\varepsilon} \partial_{t} u^{\varepsilon}, A_{0}^{-1} \partial_{t} q^{\varepsilon}\right)\right| & =\left|\left(A_{\varepsilon} \partial_{t} u^{\varepsilon},\left(A_{\varepsilon}^{-1}-A_{0}^{-1}\right) \partial_{t} q^{\varepsilon}\right)\right| \leq\left\|A_{\varepsilon} \partial_{t} u^{\varepsilon}\right\|\left\|A_{\varepsilon}^{-1}-A_{0}^{-1}\right\|_{\mathcal{L}\left(L^{2}(\Omega)\right)}\left\|\partial_{t} q^{\varepsilon}\right\| \\
& \leq M\left\|A_{\varepsilon}^{-1}-A_{0}^{-1}\right\|_{\mathcal{L}\left(L^{2}(\Omega)\right)}\left\|\partial_{t} q^{\varepsilon}\right\|_{H^{-1}(\Omega)}^{1 / 2} \\
& \leq M\left(\frac{3}{4}\left\|A_{\varepsilon}^{-1}-A_{0}^{-1}\right\|_{\mathcal{L}\left(L^{2}(\Omega)\right)}^{4 / 3}+\frac{1}{4}\left\|\partial_{t} q^{\varepsilon}\right\|_{H^{-1}(\Omega)}^{2}\right) .
\end{aligned}
$$


3. From (2) we can replace (5.12) with

$$
\frac{d}{d t} \Lambda \leq M_{1} e^{K t}\left\|A_{\varepsilon}^{-1}-A_{0}^{-1}\right\|_{\mathcal{L}\left(L^{2}(\Omega)\right)}^{4 / 3}+M_{2} \Lambda, \quad \Lambda:=\frac{1}{2}\left(\partial_{t} q^{\varepsilon}, A_{0}^{-1} \partial_{t} q^{\varepsilon}\right)+\frac{1}{2}\left\|q^{\varepsilon}\right\|^{2},
$$

which then leads to the desired result.

In order to further improve (5.8) (or rather (D.1)), and achieve the optimal bound with power one, we intend to argue as in the proof of Theorem 4.2. For this reason, we require additional regularity on the initial data $\xi$. In particular, we shall show that it is sufficient for $\xi \in \mathcal{E}_{\varepsilon}^{2}$ to be such that the solution $u^{\varepsilon}$ to (0.1) (with initial data $\xi$ ) satisfies

$$
\left\|A_{\varepsilon} \partial_{t}^{2} u^{\varepsilon}\right\| \leq M, \quad t \geq 0 .
$$

Then, we shall demonstrate that this additional regularity is 'natural' in the sense that the global attractor $\mathcal{A}^{\varepsilon}$ possesses such smoothness under the additional mild assumption on the non-linearity $f$ :

$$
f \in C^{3}(\mathbb{R}), \quad\left|f^{\prime \prime \prime}(s)\right| \leq K_{6}, \quad s \in \mathbb{R} .
$$

Let us introduce the mapping

$$
A u:=-\operatorname{div}(a \nabla u)
$$

recall

$$
\left\{\begin{array}{l}
\mathcal{E}^{2}=\left\{\xi \in\left(H_{0}^{1}(\Omega)\right)^{2} \mid\left(A \xi^{1}-g\right) \in H_{0}^{1}(\Omega) \text { and } A \xi^{2} \in L^{2}(\Omega)\right\} \\
\|\xi\|_{\mathcal{E}^{2}}^{2}=\left\|A \xi^{1}-g\right\|_{H_{0}^{1}(\Omega)}^{2}+\left\|A \xi^{1}\right\|^{2}+\left\|A \xi^{2}\right\|^{2},
\end{array}\right.
$$

and introduce

$$
\left\{\begin{array}{l}
\mathcal{E}^{3}:=\left\{\xi \in \mathcal{E}^{2} \mid A\left(A \xi^{1}+f\left(\xi^{1}\right)-g\right) \in L^{2}(\Omega) \text { and } A \xi^{2} \in H_{0}^{1}(\Omega)\right\}, \\
\|\xi\|_{\mathcal{E}^{3}}^{2}:=\left\|A\left(A \xi^{1}+f\left(\xi^{1}\right)-g\right)\right\|^{2}+\left\|\nabla A \xi^{2}\right\|^{2}+\|\xi\|_{\mathcal{E}^{2}}^{2} .
\end{array}\right.
$$

Our first result is that a dissipative estimate holds in $\varepsilon^{3}$.

Theorem D.1. Assume (H1) and (H3). Then for any initial data $\xi \in \mathcal{E}^{3}$ the energy solution $u$ to problem (1.2) is such that $\xi_{u} \in L^{\infty}\left(\mathbb{R}_{+} ; \mathcal{E}^{3}\right)$ and the following dissipative estimate is valid:

$$
\left\|\partial_{t}^{4} u(t)\right\|+\left\|\partial_{t}^{3} u(t)\right\|_{H_{0}^{1}(\Omega)}+\left\|A \partial_{t}^{2} u(t)\right\|+\left\|\xi_{u}(t)\right\|_{\mathcal{E}^{3}} \leq M\left(\|\xi\|_{\mathcal{E}^{3}}\right) e^{-\beta t}+M(\|g\|), \quad t \geq 0
$$

for some non-decreasing function $M$ and constant $\beta>0$ that depend only on $v>0$.

Proof. We begin by noting that since $\xi \in \mathcal{E}^{2}$ then, by the dissipative estimate in $\mathcal{E}^{2}$ (Theorem 2.5), $\xi_{u}(t):=$ $S(t) \xi$ satisfies

$$
\left\|\partial_{t}^{3} u(t)\right\|+\left\|\nabla \partial_{t}^{2} u(t)\right\|+\left\|\xi_{u}(t)\right\|_{\mathcal{E}^{2}} \leq M\left(\|\xi\|_{\mathcal{E}^{2}}\right) e^{-\beta t}+M(\|g\|), \quad t \geq 0 .
$$

In particular, we have

$$
\|u(t)\|_{C^{\alpha}(\bar{\Omega})}+\left\|\partial_{t} u(t)\right\|_{C^{\alpha}(\bar{\Omega})} \leq M\left(\|\xi\|_{\mathcal{E}^{2}}\right) e^{-\beta t}+M(\|g\|), \quad t \geq 0,
$$

where $\alpha$ is given in Remark 2.1.

Now upon differentiating (1.2), in time, three times we deduce that $r(t):=\partial_{t}^{3} u(t)$ solves the equation

$$
\partial_{t}^{2} r+y \partial_{t} r+A r=-f^{\prime \prime \prime}(u)\left(\partial_{t} u\right)^{3}-3 f^{\prime \prime}(u) \partial_{t} u \partial_{t}^{2} u-f^{\prime}(u) \partial_{t}^{3} u=: F(t), \quad t \geq 0,
$$

with initial data

$$
r(0)=y^{2} \xi^{2}+y\left(A \xi^{1}+f\left(\xi^{1}\right)-g\right)-A \xi^{2}-f^{\prime}\left(\xi^{1}\right) \xi^{2}
$$

and

$$
\partial_{t} r(0)=-y r(0)+y A \xi^{2}+A\left(A \xi^{1}+f\left(\xi^{1}\right)-g\right)-f^{\prime \prime}\left(\xi^{1}\right)\left(\xi^{2}\right)^{2}+f^{\prime}\left(\xi^{1}\right)\left(y \xi^{2}+A \xi^{1}+f\left(\xi^{1}\right)-g\right) .
$$


Now by (D.2) and (D.3) we readily deduce that $F \in L^{\infty}\left(\mathbb{R}_{+} ; L^{2}(\Omega)\right)$. Additionally, since $\xi \in \mathcal{E}^{3}$ we see that $r(0) \in$ $H_{0}^{1}(\Omega)$ and $\partial_{t} r(0) \in L^{2}(\Omega)$, i.e. $\left(r(0), \partial_{t} r(0)\right) \in \mathcal{E}$. Consequently, by standard linear dissipative estimates for $r$, we find

$$
\left\|\partial_{t}^{4} u(t)\right\|+\left\|\partial_{t}^{3} u(t)\right\|_{H_{0}^{1}(\Omega)} \leq M\left(\|\xi\|_{\mathcal{E}^{3}}\right) e^{-\beta t}+M(\|g\|), \quad t \geq 0,
$$

for some $M$ that depends only on $v$.

Now, the remaining claims are proven by differentiating (1.2) once to get

$$
\left\|A \partial_{t} u(t)\right\|_{H_{0}^{1}(\Omega)} \leq M\left(\|\xi\|_{\mathcal{E}^{3}}\right) e^{-\beta t}+M(\|g\|), \quad t \geq 0 .
$$

Then differentiating (1.2) one more time to get

$$
\left\|A \partial_{t}^{2} u(t)\right\| \leq M\left(\|\xi\|_{\mathcal{E}^{3}}\right) e^{-\beta t}+M(\|g\|), \quad t \geq 0,
$$

and finally re-arranging (1.2) to get

$$
\|A(A u(t)+f(u(t))-g)\| \leq M\left(\|\xi\|_{\mathcal{E}^{3}}\right) e^{-\beta t}+M(\|g\|), \quad t \geq 0 .
$$

Equipped with Theorem 2.1 we are ready to prove the desired improvement of (D.1). Namely, upon setting $\varepsilon_{\varepsilon}^{3}$ to be $\varepsilon^{3}$ for the case $a=a\left(\frac{\dot{\bar{\varepsilon}}}{)}\right.$ and $B_{\mathcal{E}_{\varepsilon}^{3}}(0, R):=\left\{\xi \in \mathcal{E}_{\varepsilon}^{3} \mid\|\xi\|_{\mathcal{E}_{\varepsilon}^{3}} \leq R\right\}$, the following result holds.

Theorem D.2. Assume (H1) and (H3). Then, for every $\xi \in B_{\mathcal{E}_{\varepsilon}^{3}}(0, R)$, the following inequality

$$
\left\|\partial_{t} S_{\varepsilon}(t) \xi-\partial_{t} S_{0}(t) \Pi_{\varepsilon} \xi\right\|_{\mathcal{E}^{-1}} \leq M e^{K t}\left\|A_{\varepsilon}^{-1}-A_{0}^{-1}\right\|_{\mathcal{L}\left(L^{2}(\Omega)\right)}, \quad t \geq 0,
$$

holds for some non-decreasing functions $M=M(R,\|g\|)$ and $K=K(R,\|g\|)$ which are independent of $\varepsilon>0$.

Proof. The argument is similar to that in Theorem 4.2 so we shall just outline the main ideas.

Set $\xi_{u^{\varepsilon}}(t):=S_{\varepsilon}(t) \xi, \xi_{u^{0}}(t):=S_{0}(t) \Pi_{\varepsilon} \xi$ and recall $\xi_{0}=\Pi_{\varepsilon} \xi$. Then by the dissipative estimates for $\xi_{u^{\varepsilon}}$ in $\varepsilon_{\varepsilon}^{3}$ (Theorem D.1) and $\xi_{u^{0}}$ in $\varepsilon_{0}^{2}$ (Theorem 2.5) we have the following uniform bounds in $t$ and $\varepsilon$ :

$$
\left\|u^{\varepsilon}\right\|_{H_{0}^{1}(\Omega)}+\left\|A_{\varepsilon} \partial_{t} u^{\varepsilon}\right\|+\left\|A_{\varepsilon} \partial_{t}^{2} u^{\varepsilon}\right\|+\left\|u^{0}\right\|_{H_{0}^{1}(\Omega)} \leq M .
$$

The difference $q^{\varepsilon}:=\partial_{t} u^{\varepsilon}-\partial_{t} u^{0}$ solves

$$
\left\{\begin{array}{l}
\partial_{t}^{2} q^{\varepsilon}+y \partial_{t} q^{\varepsilon}+A_{0} q^{\varepsilon}=A_{0} \partial_{t} u^{\varepsilon}-A_{\varepsilon} \partial_{t} u^{\varepsilon}+f^{\prime}\left(u^{0}\right) \partial_{t} u^{0}-f^{\prime}\left(u^{\varepsilon}\right) \partial_{t} u^{\varepsilon}, \quad x \in \Omega, t \geq 0, \\
\left.\xi_{q^{\varepsilon}}\right|_{t=0}=\left(\xi^{2}-\xi_{0}^{2}, y\left(\xi_{0}^{2}-\xi^{2}\right)+f\left(\xi_{0}^{1}\right)-f\left(\xi^{1}\right)\right),\left.\quad q^{\varepsilon}\right|_{\partial \Omega}=0,
\end{array}\right.
$$

and we have

$$
\left\|\left.\xi_{q^{\varepsilon}}\right|_{t=0}\right\|_{\mathcal{E}^{-1}} \leq C\left\|A_{\varepsilon}^{-1}-A_{0}^{-1}\right\|_{\mathcal{L}\left(L^{2}(\Omega)\right)} .
$$

After testing the first equation in the above problem with $A_{0}^{-1} \partial_{t} q^{\varepsilon}$ and some algebra (similar to that in Theorem 4.2) we deduce that

$$
\begin{aligned}
\frac{d}{d t} \Lambda \leq-\left(A_{\varepsilon} \partial_{t}^{2} u^{\varepsilon},\left(A_{\varepsilon}^{-1}-A_{0}^{-1}\right) q^{\varepsilon}\right)+ & \left(f^{\prime}\left(u^{0}\right) \partial_{t} u^{0}-f^{\prime}\left(u^{\varepsilon}\right) \partial_{t} u^{\varepsilon}, A_{0}^{-1} \partial_{t} q^{\varepsilon}\right), \\
& \text { where } \Lambda:=\frac{1}{2}\left\|q^{\varepsilon}\right\|^{2}+\frac{1}{2}\left(\partial_{t} q^{\varepsilon}, A_{0}^{-1} \partial_{t} q^{\varepsilon}\right)-\left(A_{\varepsilon} \partial_{t} u^{\varepsilon},\left(A_{\varepsilon}^{-1}-A_{0}^{-1}\right) q^{\varepsilon}\right) .
\end{aligned}
$$

Now in the proof of Theorem 5.2 we showed that

$$
\left|\left(f^{\prime}\left(u^{0}\right) \partial_{t} u^{0}-f^{\prime}\left(u^{\varepsilon}\right) \partial_{t} u^{\varepsilon}, A_{0}^{-1} \partial_{t} q^{\varepsilon}\right)\right| \leq M_{1}\left(e^{K t}\left\|A_{\varepsilon}^{-1}-A_{0}^{-1}\right\|_{\mathcal{L}\left(L^{2}(\Omega)\right)}^{2}+\frac{1}{2}\left\|q^{\varepsilon}\right\|^{2}+\frac{1}{2}\left(\partial_{t} q^{\varepsilon}, A_{0}^{-1} \partial_{t} q^{\varepsilon}\right)\right) .
$$

Therefore

$$
\begin{aligned}
\frac{d}{d t} \Lambda \leq\left(2 M_{1} A_{\varepsilon} \partial_{t} u^{\varepsilon}-A_{\varepsilon} \partial_{t}^{2} u^{\varepsilon},\left(A_{\varepsilon}^{-1}-A_{0}^{-1}\right) q^{\varepsilon}\right)-2 M_{1}\left(A_{\varepsilon} \partial_{t} u^{\varepsilon},\left(A_{\varepsilon}^{-1}-A_{0}^{-1}\right) q^{\varepsilon}\right) \\
+M_{1}\left(e^{K t}\left\|A_{\varepsilon}^{-1}-A_{0}^{-1}\right\|_{\mathcal{L}\left(L^{2}(\Omega)\right)}^{2}+\frac{1}{2}\left\|q^{\varepsilon}\right\|^{2}+\frac{1}{2}\left(\partial_{t} q^{\varepsilon}, A_{0}^{-1} \partial_{t} q^{\varepsilon}\right)\right),
\end{aligned}
$$


and since

$$
\left|\left(2 M_{1} A_{\varepsilon} \partial_{t} u^{\varepsilon}-A_{\varepsilon} \partial_{t}^{2} u^{\varepsilon},\left(A_{\varepsilon}^{-1}-A_{0}^{-1}\right) q^{\varepsilon}\right)\right| \leq C\left\|A_{\varepsilon}^{-1}-A_{0}^{-1}\right\|_{\mathcal{L}\left(L^{2}(\Omega)\right.}^{2}+M_{1} \frac{1}{2}\left\|q^{\varepsilon}\right\|^{2},
$$

we find

$$
\frac{d}{d t} \Lambda \leq 2 M_{1} \Lambda+C e^{K t}\left\|A_{\varepsilon}^{-1}-A_{0}^{-1}\right\|_{\mathcal{L}\left(L^{2}(\Omega)\right)}^{2},
$$

from which the desired result follows.

We finish this section with the following result on the smoothness of the global attractor.

Theorem D.3. Assume (H1) and (H3), and let $\mathcal{A}$ be the global attractor of the dynamical system $(\mathcal{E}, S(t))$ given by (1.4). Then

$$
\|\mathcal{A}\|_{\mathcal{E}^{3}} \leq M(\|g\|)
$$

for some non-decreasing $M$ that depends only on $v$.

Indeed this result can be proved by arguing as in Section 2 for the following splitting: for initial data $\xi \in$ $B_{\mathcal{E}^{2}}\left(0, R_{1}\right)$ we consider $H \in H_{0}^{1}(\Omega)$ that satisfies

$$
-\operatorname{div}(a \nabla H)=-f^{\prime}\left(\xi^{1}\right) \xi^{2} \in L^{2}(\Omega),
$$

and $G \in H_{0}^{1}(\Omega)$ that satisfies

$$
-\operatorname{div}(a \nabla G)=g-f\left(\xi^{1}\right)-y H \in L^{2}(\Omega) .
$$

Then, we decompose the solution $u$ to (1.2) as $u=v+w$ where

$$
\left\{\begin{array}{l}
\partial_{t}^{2} v+y \partial_{t} v-\operatorname{div}(a \nabla v)=0, \quad x \in \Omega, t \geq 0 \\
\left.\xi_{v}\right|_{t=0}=\left(\xi^{1}-G, \xi^{2}-H\right),\left.\quad v\right|_{\partial \Omega}=0,
\end{array}\right.
$$

and

$$
\left\{\begin{array}{l}
\partial_{t}^{2} w+y \partial_{t} w-\operatorname{div}(a \nabla w)=-f(u)+g, \quad x \in \Omega, t \geq 0, \\
\left.\xi_{w}\right|_{t=0}=(G, H),\left.\quad w\right|_{\partial \Omega}=0 .
\end{array}\right.
$$

The main points to highlight are that we can argue as in the proof of Theorem 2.1 (to produce an analogue of Lemma 2.1) and establish that

$$
\operatorname{dist}_{\mathcal{E}}\left(S(t) B_{\mathcal{E}^{2}}\left(0, R_{1}\right), B_{\mathcal{E}^{3}}\left(0, R_{2}\right)\right) \leq M e^{-\beta t}, \quad t \geq 0,
$$

holds for some positive constants $R_{2}, M$ and $\beta$ that depend only on $v$. Then, we use the transitivity of exponential attraction (Theorem 2.2) and Corollary 2.1 to deduce that $B_{\mathcal{E}^{3}}\left(0, R_{2}\right)$ attracts bounded sets in $\varepsilon$ :

$$
\operatorname{dist}_{\mathcal{E}}\left(S(t) B, B_{\mathcal{E}^{3}}\left(0, R_{2}\right)\right) \leq M\left(\|B\|_{\mathcal{E}}\right) e^{-\beta t}, \quad t \geq 0 .
$$

This finally allows us to argue as in the proof of Theorem 2.4 to prove Theorem D.3.

Consequently, the improved regularity of the attractor (Theorem D.3) allows us to apply, when appropriate, the improved inequality (Theorem D.2) in obtaining error estimates in homogenisation (cf. Remark 5.2).

Acknowledgement: The authors would like to thank Sergey Zelik for helpful discussions related to this work. S. Cooper was supported by the EPSRC grant EP/M017281/1 ("Operator asymptotics, a new approach to lengthscale interactions in metamaterials"). 


\section{References}

[1] E. Sánchez-Palencia. Nonhomogeneous media and vibration theory, volume 127 of Lecture Notes in Physics. SpringerVerlag, Berlin-New York, 1980.

[2] A. V. Babin and M. I. Vishik. Attractors of evolution equations, volume 25 of Studies in Mathematics and its Applications. North-Holland Publishing Co., Amsterdam, 1992. Translated and revised from the 1989 Russian original by Babin.

[3] V. Chepyzhov and M. Vishik. Attractors for equations of mathematical physics, volume 49 of American Mathematical Society Colloquium Publications. American Mathematical Society, Providence, RI, 2002.

[4] J. K. Hale. Asymptotic behavior of dissipative systems, volume 25 of Mathematical Surveys and Monographs. American Mathematical Society, Providence, RI, 1988.

[5] A. Miranville and S. Zelik. Attractors for dissipative partial differential equations in bounded and unbounded domains. In Handbook of differential equations: evolutionary equations. Vol. IV, Handb. Differ. Equ., pages 103-200. Elsevier/NorthHolland, Amsterdam, 2008.

[6] G. R. Sell and Y. You. Dynamics of evolutionary equations, volume 143 of Applied Mathematical Sciences. Springer-Verlag, New York, 2002.

[7] R. Temam. Infinite-dimensional dynamical systems in mechanics and physics, volume 68 of Applied Mathematical Sciences. Springer-Verlag, New York, second edition, 1997.

[8] G. Chechkin, V. Chepyzhov, and L. Pankratov. Homogenization of trajectory attractors of Ginzburg-Landau equations with randomly oscillating terms. Discrete Contin. Dyn. Syst. Ser. B, 23(3):1133-1154, 2018.

[9] V. Chepyzhov, M. Conti, and V. Pata. Averaging of equations of viscoelasticity with singularly oscillating external forces. J. Math. Pures Appl. (9), 108(6):841-868, 2017.

[10] B. Fiedler and M. Vishik. Quantitative homogenization of analytic semigroups and reaction-diffusion equations with Diophantine spatial frequencies. Adv. Differential Equations, 6(11):1377-1408, 2001.

[11] M. Vishik and B. Fidler. Quantative averaging of global attractors of hyperbolic wave equations with rapidly oscillating coefficients. Uspekhi Mat. Nauk, 57(4(346)):75-94, 2002.

[12] M. Efendiev and S. Zelik. Attractors of the reaction-diffusion systems with rapidly oscillating coefficients and their homogenization. Ann. Inst. H. Poincaré Anal. Non Linéaire, 19(6):961-989, 2002.

[13] I. D. Chueshov and L. S. Pankratov. Upper semicontinuity of attractors of semilinear parabolic equations with asymptotically degenerating coefficients. Mat. Fiz. Anal. Geom., 6(1-2):158-181, 1999.

[14] L. S. Pankratov and I. D. Chueshov. Averaging of attractors of nonlinear hyperbolic equations with asymptotically degenerate coefficients. Mat. Sb., 190(9):99-126, 1999.

[15] V. V. Jikov, S. M. Kozlov, and O. A. Oleinik. Homogenization of differential operators and integral functionals. SpringerVerlag, Berlin, 1994. Translated from the Russian by G. A. Yosifian [G. A. Iosif'yan].

[16] V. V. Zhikov and S. E. Pastukhova. On operator estimates in homogenization theory. Uspekhi Mat. Nauk, 71(3(429)):27122, 2016.

[17] T. Suslina. Homogenization of the Dirichlet problem for elliptic systems: $L_{2}$-operator error estimates. Mathematika, 59(2):463-476, 2013.

[18] T. Suslina. Homogenization of the Neumann problem for elliptic systems with periodic coefficients. SIAM J. Math. Anal., 45(6):3453-3493, 2013.

[19] M. Birman and T. Suslina. Threshold effects near the lower edge of the spectrum for periodic differential operators of mathematical physics. In Alexander A. Borichev and Nikolai K. Nikolski, editors, Systems, Approximation, Singular Integral Operators, and Related Topics, pages 71-107, Basel, 2001. Birkhäuser Basel.

[20] M. Birman and T. Suslina. Periodic second-order differential operators. Threshold properties and averaging. Algebra $i$ Analiz, 15(5):1-108, 2003.

[21] M. Birman and T. Suslina. Operator error estimates for the averaging of nonstationary periodic equations. Algebra $i$ Analiz, 20(6):30-107, 2008.

[22] M. Dorodnyi and T. Suslina. Spectral approach to homogenization of hyperbolic equations with periodic coefficients. J. Differential Equations, 264(12):7463-7522, 2018.

[23] T. Suslina. Spectral approach to homogenization of nonstationary Schrödinger-type equations. J. Math. Anal. Appl., 446(2):1466-1523, 2017.

[24] Yu. Meshkova. On operator error estimates for homogenization of hyperbolic systems with periodic coefficients. arXiv: $1705.02531,2018$.

[25] S. Brahim-Otsmane, G. A. Francfort, and F. Murat. Correctors for the homogenization of the wave and heat equations. J. Math. Pures Appl. (9), 71(3):197-231, 1992.

[26] M. I. Vishik, S. V. Zelik, and V. V. Chepyzhov. Regular attractors and their nonautonomous perturbations. Mat. Sb., 204(1):3-46, 2013

[27] A. Eden, C. Foias, B. Nicolaenko, and R. Temam. Exponential attractors for dissipative evolution equations, volume 37 of RAM: Research in Applied Mathematics. Masson, Paris; John Wiley \& Sons, Ltd., Chichester, 1994. 
[28] M. Efendiev, A. Miranville, and S. Zelik. Exponential attractors for a nonlinear reaction-diffusion system in $\mathbf{R}^{3}$. C. R. Acad. Sci. Paris Sér. I Math., 330(8):713-718, 2000.

[29] M. Efendiev, S. Zelik, and A. Miranville. Exponential attractors and finite-dimensional reduction for non-autonomous dynamical systems. Proc. Roy. Soc. Edinburgh Sect. A, 135(4):703-730, 2005.

[30] P. Fabrie, C. Galusinski, A. Miranville, and S. Zelik. Uniform exponential attractors for a singularly perturbed damped wave equation. Discrete Contin. Dyn. Syst., 10(1-2):211-238, 2004. Partial differential equations and applications.

[31] V. Pata and S. Zelik. A remark on the damped wave equation. Commun. Pure Appl. Anal., 5(3):609-614, 2006.

[32] V. Maz'ya and T. Shaposhnikova. Theory of Sobolev multipliers, volume 337 of Grundlehren der Mathematischen Wissenschaften [Fundamental Principles of Mathematical Sciences]. Springer-Verlag, Berlin, 2009. With applications to differential and integral operators.

[33] T. Suslina. Homogenization of a periodic parabolic Cauchy problem in the Sobolev space $H^{1}\left(\mathbb{R}^{d}\right)$. Math. Model. Nat. Phenom., 5(4):390-447, 2010. 